\title{
The phosphatase MKP1 as a target to enhance replicative stress and apoptosis in tumor cells
}

\author{
Dissertation \\ for the award of the degree \\ “Doctor of Philosophy" (Ph.D.) \\ in the Molecular Biology program \\ at the Georg-August University Göttingen \\ Faculty of Biology
}

submitted by

Veena Jagannathan

from Little Rock, USA

Göttingen, 2015 


\section{Members of the Thesis Committee}

Prof. Dr. Matthias Dobbelstein (Reviewer)

PD Dr.Wilfried Kramer (Reviewer)

Prof. Dr. Holger Reichardt
Institute of Molecular Oncology University Medical Centre Georg-August-Universität, Göttingen

Department of General Microbiology Institute for Microbiology and Genetics Georg-August-Universität, Göttingen

Dept. of Cellular and Molecular Immunology University Medical Centre

Georg-August-Universität, Göttingen

Date of the oral examination: $6^{\text {th }}$ May, 2015 


\section{Affidavit}

I hereby declare that the presented thesis entitled "The phosphatase MKP1 as a target to enhance replicative stress and apoptosis in tumor cells" has been written independently and with no other sources and aids than quoted.

Gottingen, $31^{\text {st }}$ March 2015

Veena Jagannathan 


\section{Dedication}

This thesis is dedicated in the loving memory of my aunt, Late Mrs. Sundari Santhanam, who fought but lost her battle against cancer.

....Perima, you have been a great source of inspiration to all of us in the family. I have always looked up to you and even though you are not present amongst us physically, your memories have given me the strength to pick myself up and perform better. Your will power, fighting spirit, sincerity and dedication was something that I have always strived to achieve in whatever I do. You will be there forever in our hearts, perima. We love you a lot!

This thesis is also dedicated to all the others who still wake up every day to fight this disease.

Together with our efforts, I believe we can make their wait worth fighting for. 


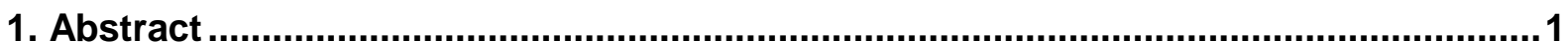

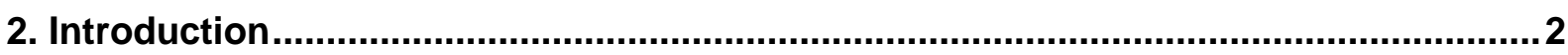

2.1 Ready, set and fire! : Initiating DNA replication ................................................... 2

2.1.1 Are you licensed yet?: Forming the pre-Replicative Complex.............................. 3

2.1.2 Pulling the trigger: A kinase mediated activation of pre-RC ............................. 4

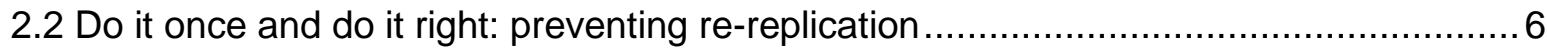

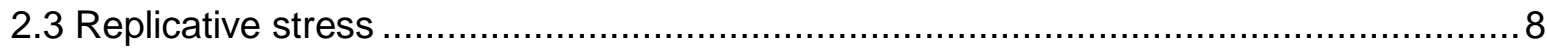

2.4 Replication Checkpoint: 'Check'mating DNA damage at the fork .......................... 8

2.4.1 Switch on those kinases: Activating the ATM and ATR signaling ...................... 8

2.4.2 Three's company: p38-MK2 joins the DDR network .................................... 10

2.4.3 Inhibit globally but activate locally: Regulating replication initiation after stress .... 11

2.4.4 Mending the ends: Repair and restart of stalled replication forks ..................... 13

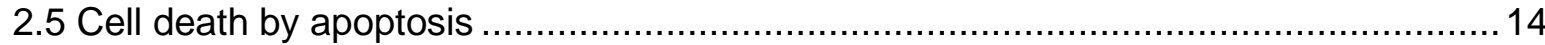

2.6 Cancer and chemotherapy: DNA replication as a double edged sword ....................16

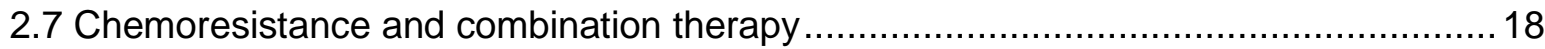

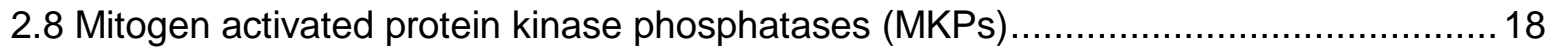

2.8.1 Switching off the MAPK signaling by mitogen activated protein kinase phosphatase

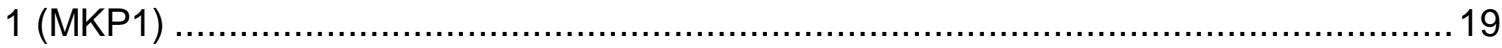

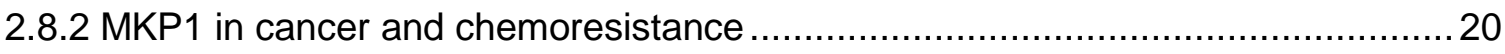

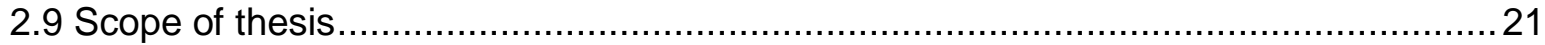

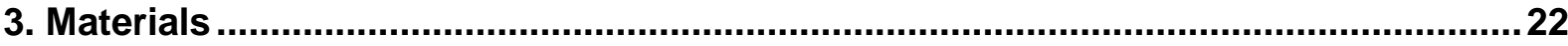

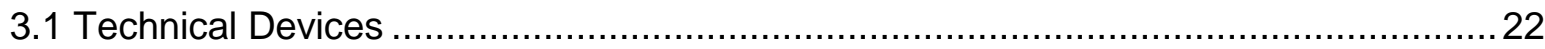

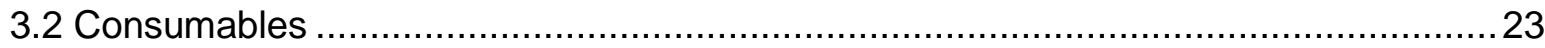

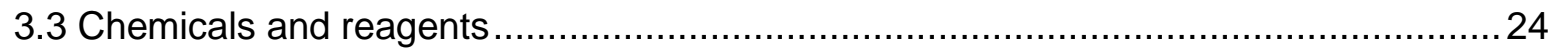

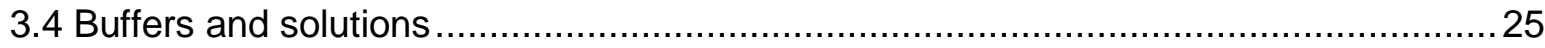

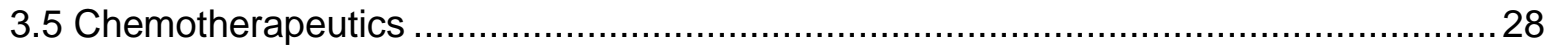

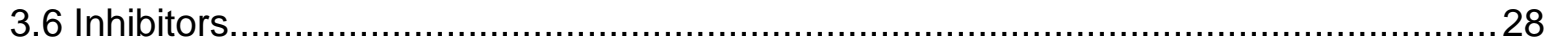

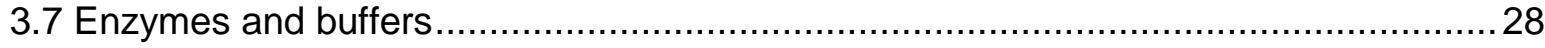

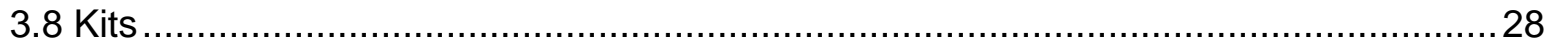

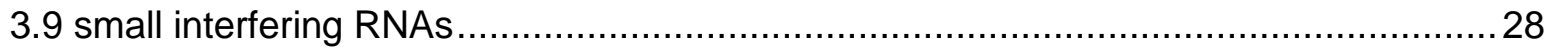

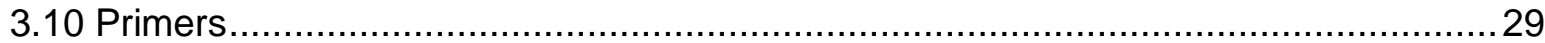

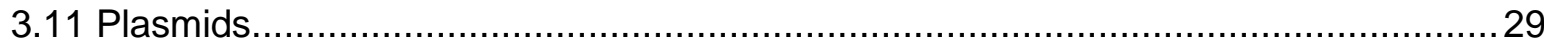

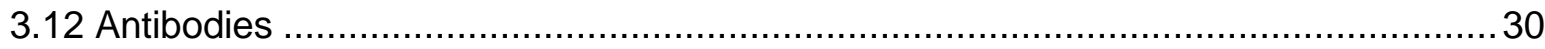

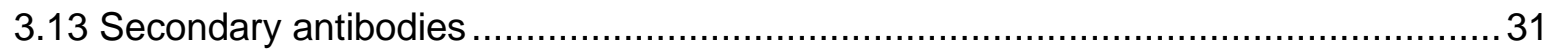


3.14 Human cell lines 31

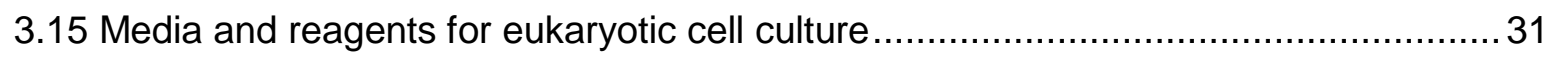

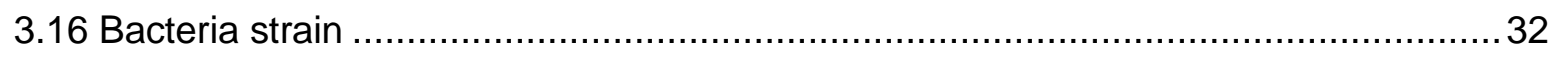

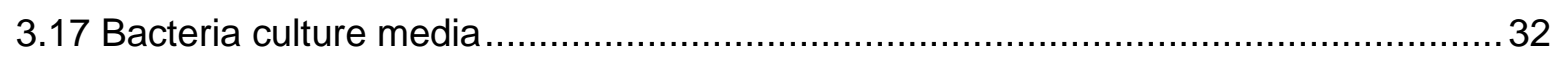

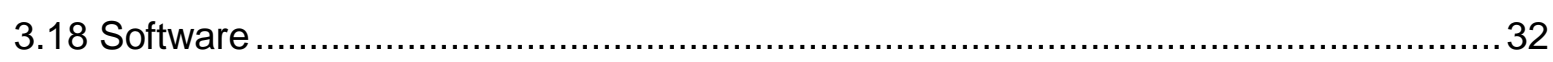

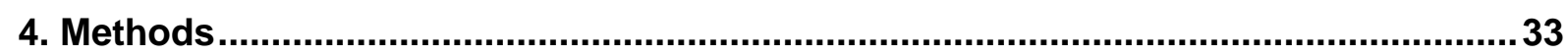

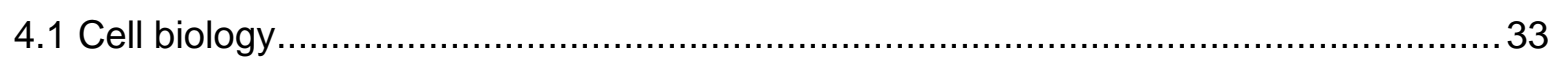

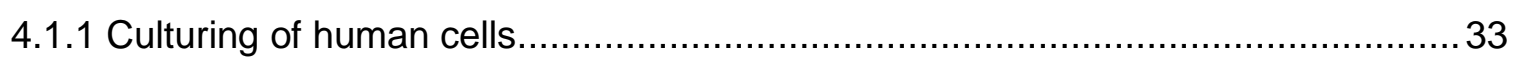

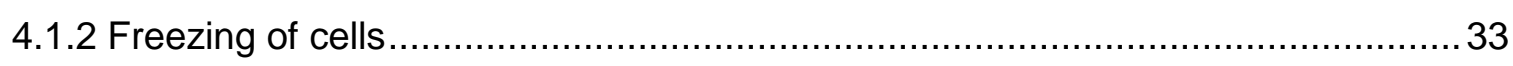

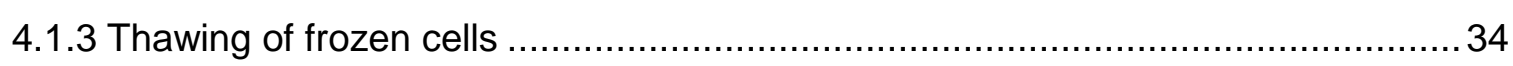

4.1.4 Inhibitor and chemotherapeutic treatment ................................................ 34

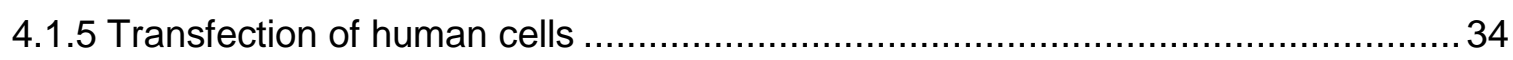

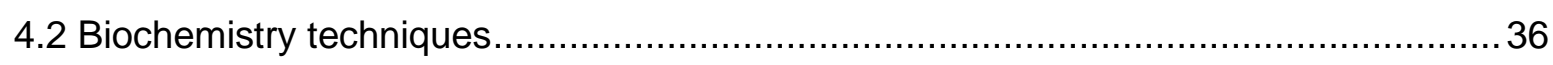

4.2.1 Preparation of cell lysates for protein separation by SDS-PAGE ......................... 36

4.2.2 Bicinchoninic acid (BCA) assay for protein estimation.................................... 36

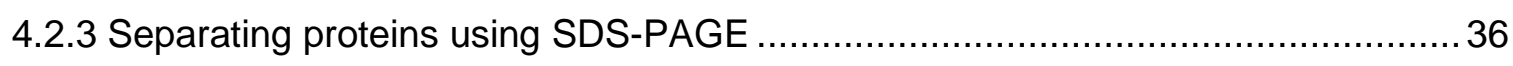

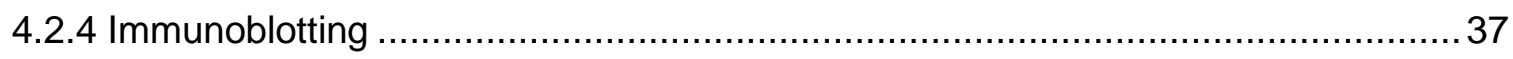

4.2.5 Quantitative Reverse Transcription- Polymerase Chain Reaction (qRT-PCR)...... 38

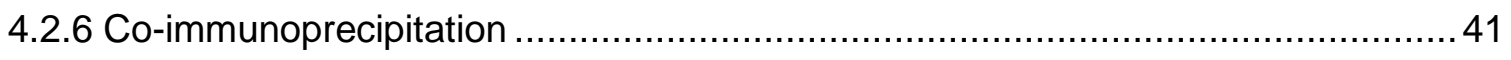

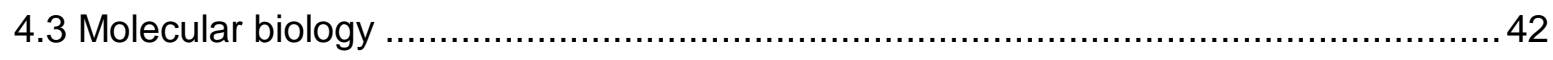

4.3.1 Heat-shock transformation of chemical competent bacteria ............................. 42

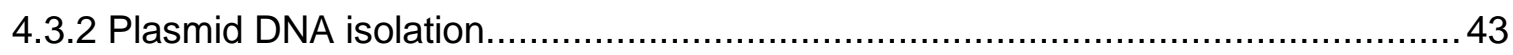

4.3.3 Measurement of nucleic acid concentration .............................................. 43

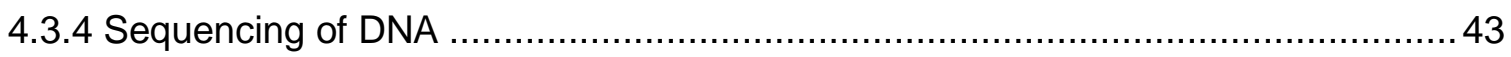

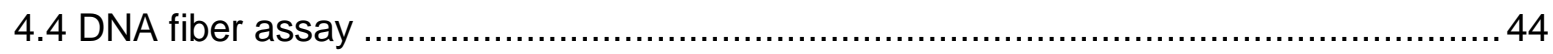

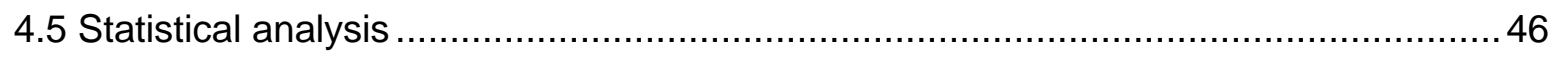

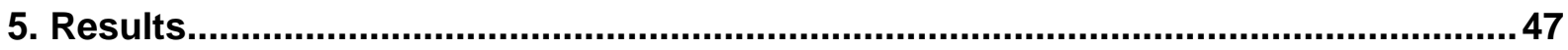

5.1 Inhibition of MKP1, using $\mathrm{BCl}$, accumulates phospho- $\mathrm{H} 2 \mathrm{AX}$ in various cell lines....... 47

5.2 MKP1 inhibition impairs replication fork progression....................................... 48

5.3 MKP1 inhibitor acts together with Gemcitabine to further reduce replication fork speed

5.4 Inhibition of MKP1 differentially regulates checkpoint signaling ................................5 52

5.5 Activation of ATM and MK2, is responsible for replicative stress induced by MKP1

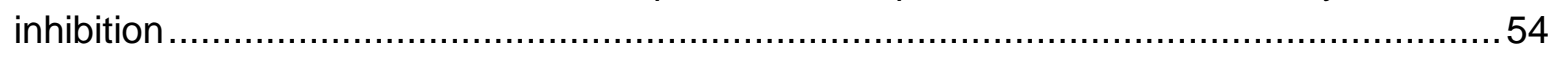

5.6 MKP1 inhibition decreases origin firing, independently of ATM activity .....................57

5.7 Inhibiting MKP1 activity results in the loss of replication initiator kinase Cdc7 and MCM2 phosphorylation ...... 
5.8 Proteasome degradation or mRNA regulation are not responsible for the loss of Cdc7 after MKP1 inhibition

5.9 Longer exposure to $\mathrm{BCl}$ or reduction of MKP1 levels leads to apoptosis

5.10 MKP1 knockdown is accompanied by a caspase independent degradation of an antiapoptotic protein, Mcl-1

5.11 Apoptosis upon MKP1 depletion can be partially rescued by Mcl-1 over-expression 66

5.12 MKP1 interacts with Mcl-1 under unstressed conditions 67

6. Discussion. 68

6.1 MK2 in controlling replication events upon MKP1 inhibition 69

6.2 ATM: Deciphering the code in replication fork progression 70

6.3 The disappearance of Cdc7. .73

6.4 Keep them close for survival: MKP1 and Mcl-1. 75

6.5 Chemosensitization with MKP1 inhibition 76

6.6 Conclusions: MKP1 in DNA replication, cellular survival and cancer 77

7. Appendix .79

8. Abbreviations. 83

9. References 87

Acknowledgements 99

Curriculum vitae 102 


\section{List of Figures}

Figure 2.1 Formation of the pre-Replicative Complex. ................................................ 3

Figure 2.2 Events mediating the activation of pre-RC. ................................................ 5

Figure 2.3 Events preventing re-replication. ............................................................. 7

Figure 2.4 Activation of replication checkpoint signaling. ............................................ 10

Figure 2.5 Dormant origin firing to promote replication under stress. ............................. 12

Figure 2.6 A schematic depicting the role of MKP1 ...................................................... 19

Figure 4.1 Representative images of the various structures analyzed in DNA fiber assay ... 45

Figure 5.1 Accumulation of phospho-H2AX (S139) upon MKP1 inhibition using BCl. ......... 47

Figure 5.2 MKP1 inhibition reduces replication fork speed........................................ 49

Figure $5.3 \mathrm{BCl}$ and Gemcitabine co-operate to further impair the progression of replication

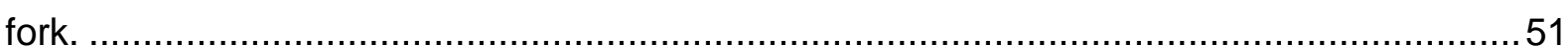

Figure 5.4 Activity of checkpoint kinases is subjected to differential regulation by MKP1.... 53 Figure 5.5 Replicative stress induced by MKP1 inhibition is dependent on ATM, but not MK2.

Figure 5.6 An ATM independent decrease in origin firing upon MKP1 inhibition................... 58

Figure 5.7 MKP1 inhibition leads to a decrease in Cdc7 levels and MCM2 phosphorylation. 60 Figure 5.8 Disappearance of Cdc7 upon MKP1 inhibition is not due to a loss of mRNA or protein.

Figure 5.9 MKP1 knockdown or BCl treatment for long hours leads to apoptosis. 63

Figure 5.10 Anti-apoptotic protein Mcl-1 is degraded independent of caspase activity upon MKP1 depletion. 65

Figure 5.11 Apoptotic effects of MKP1 knockdown are partially rescued by over-expression of $\mathrm{Mcl}-1$

Figure 5.12 MKP1 interacts with Mcl-1 in an in-vitro co-immunoprecipitation assay.....

Figure 6.1 A hypothetical model representing the role of MKP1 in replicative stress and cell survival.

Figure 7.1 MK2 inhibition rescues the accumulation of phospho-H2AX (S139) upon MKP1 inhibition.

Figure 7.2 The loss of Chk1 phosphorylation upon MKP1 inhibition and Gemcitabine treatment is independent of ATR and Claspin.

Figure 7.3 Different antibodies to Cdc7 yield similar patterns upon MKP1 inhibition. 80 Figure 7.4 Activation of p53 may be responsible for the loss of Cdc7 observed with MKP1 inhibition. 80

Figure 7.5 MKP1 knockdown leads to the activation of JNK. 81

Figure 7.6 Plasmid maps of pCMV-DUSP1 and pcDNA3. 82 


\section{List of Tables}

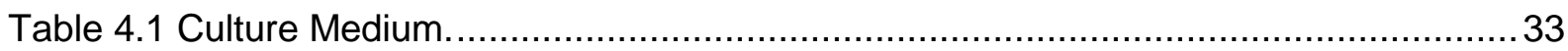

Table 4.2 Concentrations of inhibitors and chemotherapeutics used in cell culture............. 34

Table 4.3 Concentration and volume of siRNA and lipofectamine used for transient

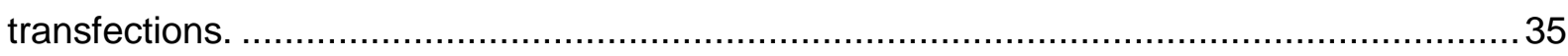

Table 4.4 Concentration and volume of siRNA and lipofectamine used for transient

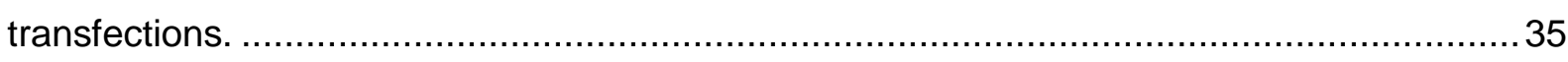

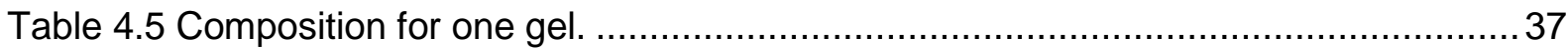

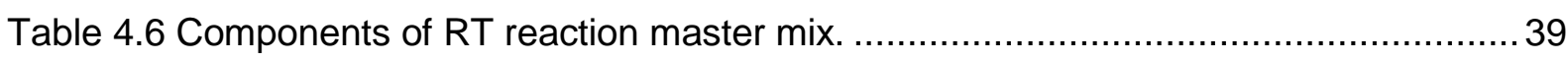

Table 4.7 Components of qPCR mix for one sample................................................... 40

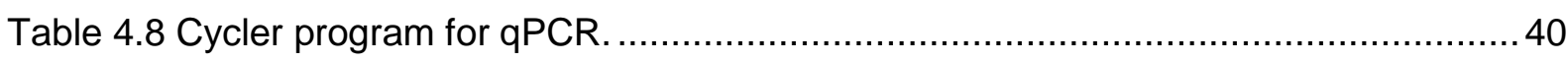

Table 4.9 Amount of plasmids used for transient transfections in Co-IP ............................ 41

Table 4.10 Cycler program for sequencing PCR ................................................... 43 


\section{Abstract}

DNA replication is a tightly regulated elementary process that ensures the exact duplication and transfer of genetic information to the next generation. However, a wide range of exogenous and endogenous genotoxic insults often impair the progression of a replication fork and give rise to a phenomenon termed as replicative stress. In such a scenario, it is imperative for cells to maintain their DNA integrity to prevent genomic instability that may lead to tumorigenesis. This is achieved by instigating the DNA damage response (DDR), a highly organized, enzyme-based signaling cascade. While the contribution of kinases in this network has been very well studied, less is known about the role of their negative regulators, the phosphatases, in the same. Hence, our studies were aimed at investigating the function of a dual-specificity phosphatase, MKP1 (alias DUSP1), in both DNA replication and DDR. Identified as a MAP kinase phosphatase, MKP1 preferentially de-phosphorylates and inactivates p38MAPK and JNK, and protects the cell from stress-induced apoptosis.

Our studies show that MKP1 inhibition accumulates phospho $\mathrm{H} 2 \mathrm{AX}(\mathrm{\gamma H} 2 \mathrm{AX})$ and activates the DDR, even in the absence of any exogenous DNA damage. This effect was attributed to a previously unknown role of MKP1 in regulating DNA replication. Using the DNA fiber assay, we could demonstrate impaired replication fork progression and reduced origin firing upon MKP1 inhibition. Moreover, in the presence of an external replicative stress stimulus, gemcitabine, MKP1 inhibition was able to further down-regulate the speed of progressing forks. Additional investigations identified the activation of checkpoint kinases, MK2 and ATM, to be responsible for mediating these replication fork defects. Besides this, prolonged inhibition or transient depletion of MKP1 led to a massive induction of apoptosis, indicating a crucial function of this phosphatase in cellular survival. Cell death was accompanied by the degradation of an anti-apoptotic protein, Mcl-1, in the MKP1 deficient cells. Furthermore, for the first time, we detected a physical interaction between these proteins in an in-vitro coimmunoprecipitation assay.

Taken together, our findings indicate that MKP1 is required for efficient DNA replication and cellular survival. Furthermore, this protective nature of MKP1 can be exploited by pharmacological antagonists in various cancers which over-express this phosphatase. Thus, we propose MKP1 as an attractive druggable target in cancer therapy, inhibition of which can enhance replicative stress and promote cell death. 


\section{Introduction}

Owing to the enormity of human genome, it is of utmost importance to ensure the faithful and complete replication of DNA, for an accurate transmission of genetic information to the next generation (Remus and Diffley 2009). A failure to do so, can damage DNA by introducing single strand nicks, double strand breaks or abnormal DNA structures (Jones and Petermann 2012). These damages, if left unresolved can result in genomic instability and hence uncontrolled cellular proliferation; or in extreme cases, activate programmed cell death (Norbury and Zhivotovsky 2004). Nonetheless, cells have devised two intelligent strategies to combat this kind of situation: first, by 'avoiding' the occurrence of such an event by making certain that the parental DNA is replicated exactly once per cell cycle, thus, eliminating the production of spontaneous DNA damage arising due to over / under replication; and second, by initiating signaling responses often mediated by kinases, to arrest the cell cycle and repair damaged DNA when needed, thereby 'overcoming' the problem.

Since not all studies are conducted using human cells as model systems, the following sections will give a brief description of DNA replication and its regulation under various stressful conditions, based on findings from Xenopus and mammalian systems.

\subsection{Ready, set and fire! : Initiating DNA replication}

Unlike in prokaryotes, where replication initiates at a fixed position on the DNA (originC or oriC), studies in metazoans revealed the existence of not one, but several such sites randomly distributed throughout the genome (Leonard and Mechali 2013). Strikingly, the metazoan 'origins' of replication - the sites where DNA starts to unwind, also lacked a strong consensus sequence when compared to its prokaryotic counterpart (Gilbert 2004, Machida, Hamlin et al. 2005). Additionally, in metazoans, concurrently firing adjacent origins are organized into groups called 'replicon clusters' (where a replicon is the size of DNA replicated by one origin), whose activation is spatio-temporally regulated. This regulation is thought to be largely dependent on the chromatin organization surrounding these clusters. While euchromatin is replicated early on in the S-phase due to its open, conducive environment; origins present in the unfavorable regions of heterochromatin fire during the later stages. Despite this distinction among origins, the requirement of initiator proteins to aid replication remains conserved throughout evolution (Jackson and Pombo 1998, Mendez and Stillman 2003, Gillespie and Blow 2010). 


\subsubsection{Are you licensed yet?: Forming the pre-Replicative Complex}

Even before DNA is duplicated, initiator proteins start assembling at the origins and 'license' DNA for replication during the late mitosis and G1 stage of cell cycle. This involves the

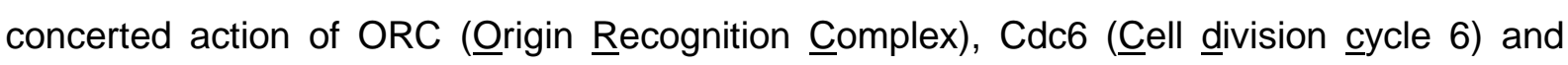
Cdt1 (Chromatin licensing and DNA replication factor 1 ) to recruit the hexameric, ring shaped minichromosome maintenance2-7 complex (MCM2-7). Among these, ORC, a six subunit complex (also called ORC1-6), is responsible for recognizing and binding to the origins (Rowles, Chong et al. 1996, Mendez and Stillman 2000). Cdc6 and Cdt1, on the other hand are recruited to the DNA in an ORC-dependent manner (Lei and Tye 2001, Blow and Hodgson 2002).

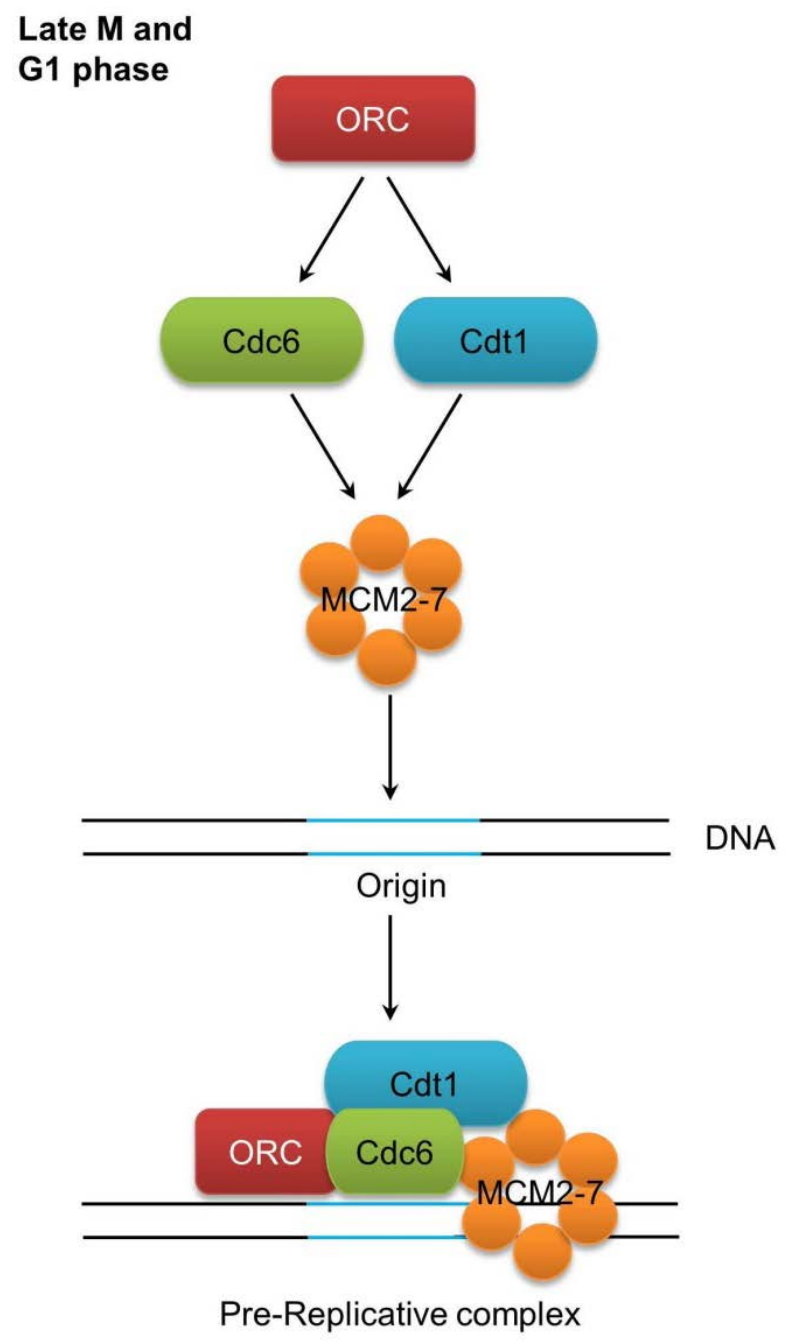

Figure 2.1 Formation of the pre-Replicative Complex.

In the late mitosis and G1-phase of the cell cycle, initiator proteins including ORC, Cdc6 and Cdt1 are sequentially loaded on the origin(s) (marked in blue). Subsequently, the helicase, MCM2-7 complex is loaded, facilitated by its interaction with Cdt1. This forms the 'pre-Replicative Complex' (pre-RC) where the MCM2-7 complex is still inactive. Figure adapted with modifications from (Machida, Hamlin et al. 2005). 
Further experiments conducted in Xenopus unveiled the presence of Cdc6 on the origin, to be a pre-requisite for Cdt1 activity (Tsuyama, Tada et al. 2005). This sequential binding of ORC, Cdc6 and Cdt1 is followed by the loading of MCM2-7 helicase, which is facilitated by its interaction with Cdt1 (Cook, Chasse et al. 2004). Moreover, ATP binding and hydrolysis by the AAA+ ATPase family proteins ORC and Cdc6 might provide the necessary energy to either assemble the six subunits of MCM on the origin or open the preformed MCM hexamer and clamp it around the DNA (Gillespie, Li et al. 2001, Mendez and Stillman 2003). This forms the pre-Replicative Complex (pre-RC); an inactive replication machinery, ready for activation in the S-phase.

\subsubsection{Pulling the trigger: A kinase mediated activation of pre-RC}

Even though all potential origin sites are licensed, not all licensed origins fire in one cell cycle. With no strict predilection, origin firing within replicon clusters seems to involve a substantial degree of stochasticity (i.e. randomness) (Lei and Tye 2001, Blow, Ge et al. 2011). Upon the onset of S-phase, two Ser/Thr kinases, namely, Cdk2 (Cyclin dependent kinase 2) and Cdc7 (eell division cycle 7), get activated by associating with their respective

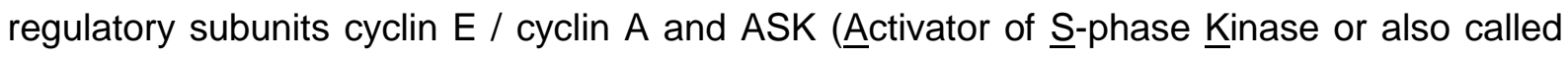
Dbf4). Besides cyclin binding, Cdk2 is phosphorylated at Thr 160 by CAK ( $\underline{C} d k \underline{A c t i v a t i n g}$ Kinase) and dephosphorylated at Thr 14 and / Tyr 15 by a dual specificity phosphatase, Cdc25A to achieve full activation. The inhibitory phosphorylation at Tyr 15 is added by a kinase, Wee1, which keeps the constitutively expressed Cdk2 inactive until an appropriate stimulus for entry to S-phase is received (Watanabe, Broome et al. 1995, Sørensen and Syljuåsen 2012). The occurrence of such post translational modifications for Cdc7 has not yet been thoroughly investigated.

Once fully functional, these S-phase kinases together promote the phosphorylation of MCM2-7 complex. In particular, phosphorylation of various sites in the $\mathrm{N}$-terminal region of MCM2 and MCM4 has been identified to assist replication initiation; either by increasing the ability to interact with and load the MCM helicase co-factor Cdc45, or by inducing a conformational change activating its helicase function (Montagnoli, Valsasina et al. 2006, Tsuji, Ficarro et al. 2006). Besides this, another adaptor protein, TOPBP1, interacts with and positions DNA polymerase, pol E, (and subsequently pol D) onto origins. This replication machinery thus assembled is called a 'replisome', which is active. Since every origin when fired emanates two replication forks moving away from each other (and the origin); two replisomes are assembled in the opposite orientation. As MCM propels along the helix, it utilizes the energy of ATP hydrolysis to unwind the parental DNA and in the process, 
generates stretches of single stranded DNA ( $\underline{s}$ DNA) which serve as templates for DNA polymerases. In this manner, replication continues until it meets another fork and terminates.

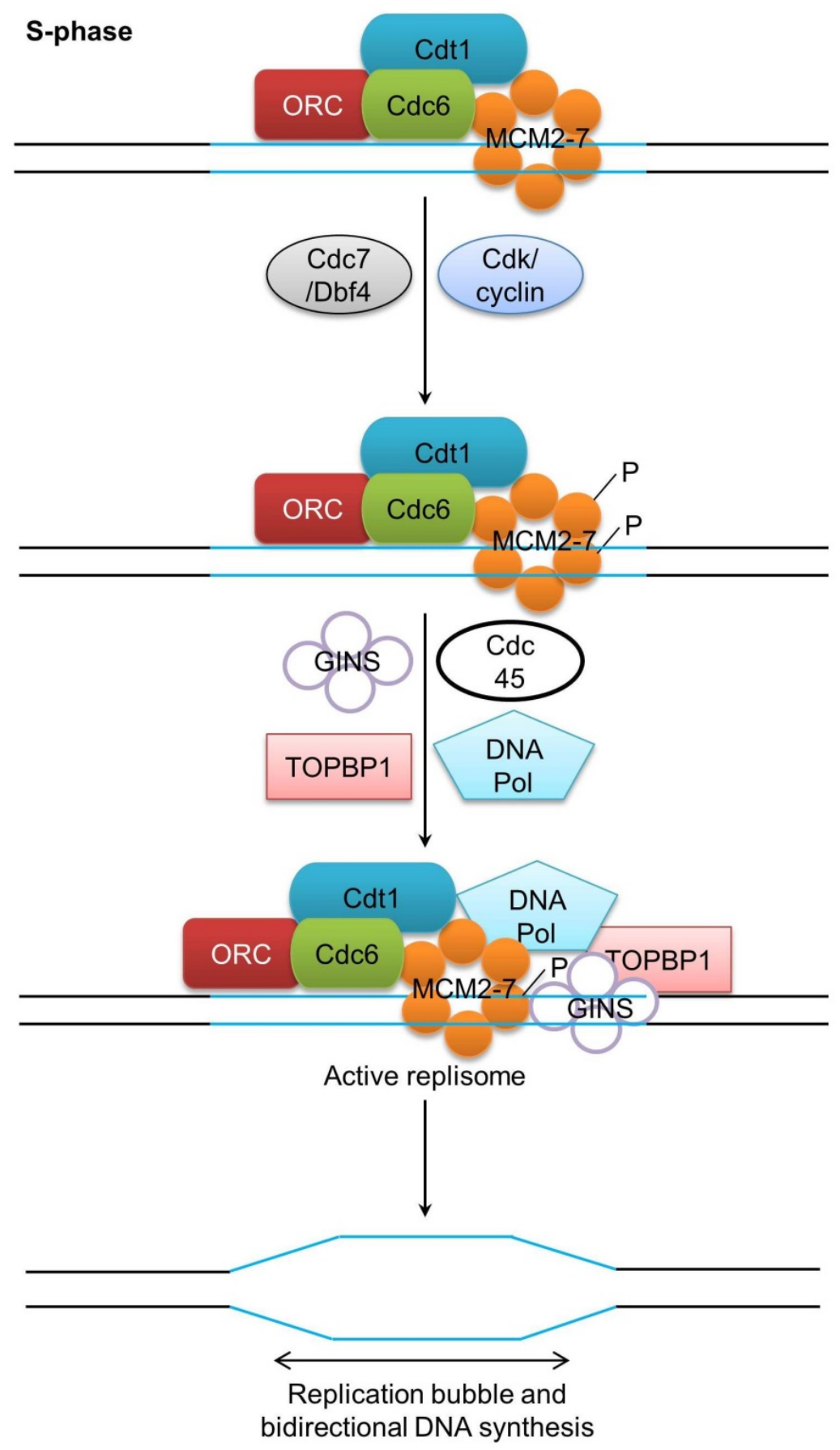

Figure 2.2 Events mediating the activation of pre-RC.

Upon entry into S-phase, the pre-RC complex on the origins is activated. Cyclin/Cdk and Cdc7/Dbf4 kinase complexes promote the phosphorylation and activation of MCM2-7 complex. This is followed by the loading of helicase co-factor Cdc45 and two other adaptor proteins, GINS and TOPBP1. This facilitates the loading of DNA polymerase and a 'replisome' is formed. The MCMs start unwinding the DNA and a replication bubble is formed. Replication then proceeds bi-directionally. In principle, two replisomes are required on one origin, only one is shown here for convenience. Figure adapted with modifications from (Machida, Hamlin et al. 2005). 
It should be noted that additional factors such as GINS complex (required for holding Cdc45MCM together), RFC-PCNA (clamp loader-sliding clamp complex, for holding DNA polymerase onto the DNA), RPA (single strand DNA binding protein) and certain histone chaperones (for chromatin remodeling) also constitute an active part of the replisome, whose details are beyond the scope of this thesis. There might still be many other factors involved which are yet to be discovered (lizuka and Stillman 1999, Kubota, Takase et al. 2003, Majka and Burgers 2004).

\subsection{Do it once and do it right: preventing re-replication}

When a new strand of DNA is being synthesized, a new origin is also in the making. Since MCM activity is indispensable for starting replication, origin re-firing is avoided by restricting the assembly and activation of pre-RC (and so $\mathrm{MCM}$ ), in two non-overlapping phases of the cell cycle. In G1, MCM2-7 present on the origins is kept passive (inactive) due to low Cdk activity. Once cells enter S-phase, a fully functional Cdk2 not only activates MCMs and initiates DNA synthesis, but at the same time also prevents re-replication by inhibiting the loading of additional MCMs through inactivation of all the pre-RC components.

Phosphorylation of Cdc6 by Cdk, followed by its proteasomal mediated degradation, is the major mechanism of restraining replication to one cycle in yeast. Contrastingly, mammalian Cdc6 when phosphorylated by cyclinA-Cdk2; is exported out of the nucleus in a Crm-1 dependent manner (Saha, Chen et al. 1998, Petersen, Lukas et al. 1999). However, Coverly and co-workers later reported that free, phosphorylated Cdc6 falls prey to regulated proteolysis, an action which seems to require the activity of cyclinA-Cdk2 (Coverley, Pelizon et al. 2000).

Interestingly, during S-phase, both human Orc1 (the largest subunit of ORC), and Cdt1 suffer the same fate as yeast Cdc6, i.e., poly-ubiquitination by SCF ubiquitin ligase and proteolytic destruction. More so, here Cdt1 is phosphorylated at its $\mathrm{N}$-terminus and thus primed by Cdk2/Cdk4 for binding with the substrate recognition component of SCF, Skp2 (Mendez, Zou-Yang et al. 2002, Liu, Li et al. 2004). The role of Cdk2 for Orc1 in this context is still not well elucidated. Along the same lines, studies done in Chinese hamster cells disclosed the existence of an Orc1 cycle in S-to-M transition where Orc1 oscillated between mono/diubiquitinated (in S-phase) and de-ubiquitinated (in M-phase) states. This ubiquitination event led to its selective dissociation from chromatin and impaired re-incorporation into the ORC complex (Li and DePamphilis 2002).

In metazoans, Cdt1 is subjected to a second level of regulation by the replication inhibitor, geminin, where Cdt1-MCM6 contact is blocked when Cdt1 binds to geminin (Yanagi, Mizuno 
et al. 2002). A dimer by nature, geminin tethers the C-terminus of Cdt1 to its coiled coil central domain and in this way prevents the entry of MCM2-7 by steric hindrance (Lee, Hong et al. 2004). Geminin levels fluctuate through the cell cycle with its accumulation in the S, G2 and $\mathrm{M}$ phases, consistent with the inhibition of Cdt1 observed herein. To allow the formation of pre-RC during G1, Cdt1 must be relieved from the clasp of geminin, a process made possible by the ubiquitin ligase, APC (Anaphase Promoting Complex) (McGarry and Kirschner 1998).

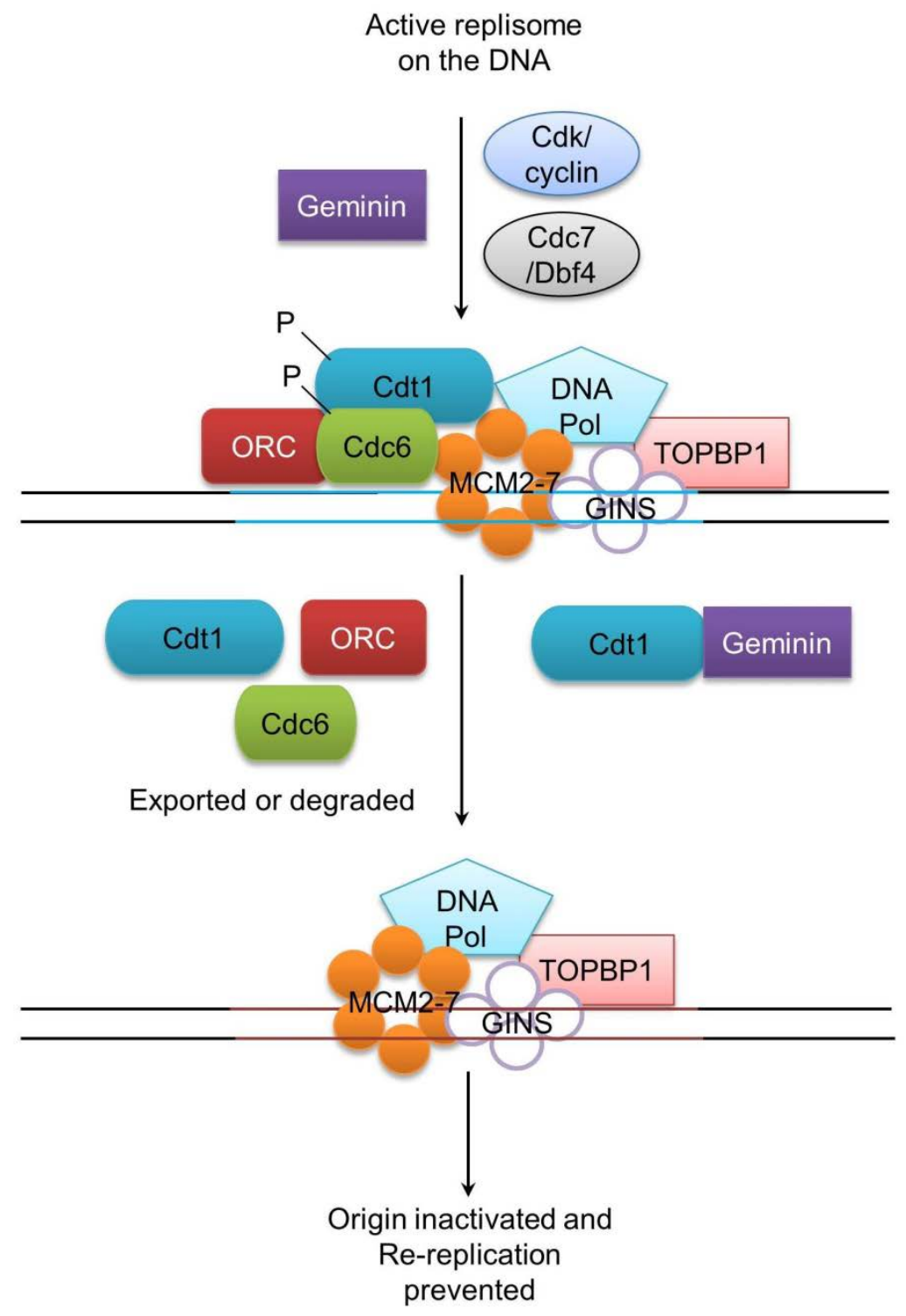

Figure 2.3 Events preventing re-replication.

The S-phase kinases complexes, apart from activating MCM complex, also phosphorylate and inactivate the components of the replisome to prevent re-firing of the origins. Cdt1 and Cdc6 are phosphorylated and marked for proteasomal degradation. Cdt1 is additionally inhibited when it binds to Geminin. Cdc6 is also exported out of the nucleus in a Crm-1 dependent manner. Orc is degraded as well, however the involvement of a phosphorylation is still not elucidated well. See text for more details. Figure adapted with modifications from (Machida, Hamlin et al. 2005). 
Besides these aforementioned mechanisms, origins are also inactivated by the process of replication itself, a newly emerging concept based on research done in Xenopus, where, Cdt1 was degraded during DNA synthesis and not initiation (Arias and Walter 2005, Takeda, Parvin et al. 2005). These mechanisms work together to safeguard genomic integrity by confining DNA replication to once and only once per cell cycle.

\subsection{Replicative stress}

It is not always smooth sailing for a replication fork in the genome, encountering various impediments along the way. This can include a wide range of obstacles generated by endogenous or exogenous means. Endogenous obstacles may include - spontaneously occurring DNA damage in the form of chemically modified bases, broken DNA or abasic sites; difficult to replicate secondary structures; long tandem repeats and DNA bound proteins. Here, DNA damage is most likely generated due to reactive oxygen species, reactive carbonyl species etc. which are common by products of cellular metabolic pathways (De Bont and van Larebeke 2004). On the other hand, various exogenous insults such as ultraviolet and ionizing irradiation, nucleoside analogs and topoisomerase inhibitors; can also contribute to the DNA damage (Espinosa, Zamora et al. 2003, Herrlich, Karin et al. 2008). Under such circumstances, the replisome does not move further and instead comes to a temporary halt. This results in replication fork stalling, until the barrier is eliminated. However, in certain cases, when a fork remains stalled for a long time, the replication machinery can get inactivated or fall off the DNA, giving rise to a collapsed fork, which is unable to restart even after the blockade is removed. The precise signaling mechanisms which help cells to deal with such stalling induced replicative stress constitute the replication checkpoint, explained in detail below (Bartek, Lukas et al. 2004, Zeman and Cimprich 2014).

\subsection{Replication Checkpoint: ‘Check’mating DNA damage at the fork}

Cellular DNA can be damaged during any phase of the cell cycle by various agents (see 2.3). To overcome this threat, cells elicit the DNA damage response (DDR) which is a complex set of signaling pathways orchestrated by various kinases like ATR, ATM, Chk1 and Chk2. Particularly during S-phase, when replication fork progression is hampered, cells activate these kinases and induce the 'replication checkpoint' which constitutes a small branch of the DDR network.

\subsubsection{Switch on those kinases: Activating the ATM and ATR signaling}

When an ongoing replication fork meets an obstacle, DNA polymerases stall. Nevertheless, MCM helicases continue to move forward and unwind the double helix, leaving behind long 
stretches of single stranded DNA (sSDNA) that immediately get coated by RPA (Replication Protein $\underline{A}$ ) - a ssDNA binding protein (Walter and Newport 2000, Byun, Pacek et al. 2005). This sends a signal to the upstream nuclear phosphatidylionositol- $\underline{3}$ (PI-3) like Ser/Thr kinase - ATR ( stalled forks by interacting with its DNA bound partner protein, ATRIP (ATR Interacting Protein). This is followed by phosphorylation of ATR at Thr 1989 and recruitment of various accessory proteins like Rad17, Claspin and the 9-1-1 complex, all of which are required for complete activation of the ATR signaling pathway (Parrilla-Castellar, Arlander et al. 2004, Branzei and Foiani 2009).

Still another nuclear transducer kinase, ATM (ㅅtaxia Telangiectasia Mutated), commonly known as a DNA double strand break (DSB) responsive kinase, also becomes activated by ATR after replication fork blocks (Stiff, Walker et al. 2006). Functioning parallel to ATR, ATM - a dimer in unstressed conditions, gets auto-phosphorylated at Ser 1981 and dissociates into active monomers (Bakkenist and Kastan 2003). This requires the presence of various

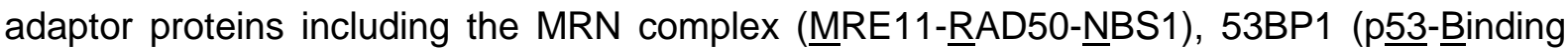

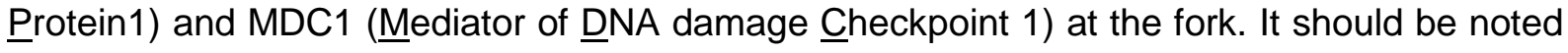
here that the mechanisms underlying the activation of ATR and ATM are still not thoroughly understood. Once activated, ATR majorly targets Checkpoint kinase 1 (Chk1) for phosphorylation at Ser 317 and Ser 345, whereas Checkpoint kinase 2 (Chk2) is phosphorylated by ATM at Thr 68 (Zhao and Piwnica-Worms 2001, Kastan and Bartek 2004). These checkpoint kinases phosphorylate and inactivate the Cdc25 phosphatases, thereby arresting the cell cycle via inhibition of Cdk-cyclin activity (Bartek and Lukas 2003, Reinhardt and Yaffe 2009).

Besides Chk1 and Chk2, ATM and ATR phosphorylate and activate many other proteins including some important ones like p53 and H2AX (Histone 2AX). The transcription factor p53, upon phosphorylation and activation, up-regulates a cyclin-Cdk inhibitor p21, and mediates cell cycle arrest at the G1/S transition. p53 is also known to facilitate apoptosis under conditions of extreme stress, for e.g., by increasing transcription of pro-apoptotic proteins (Zilfou and Lowe 2009).

On the other hand, the histone $\mathrm{H} 2 \mathrm{~A}$ variant, $\mathrm{H} 2 \mathrm{AX}$, is phosphorylated in its C-terminal tail at Ser 139 , in response to DNA damage. Initially this phospho H2AX, is localized to the site of DNA damage, but later spreads to approximately $50 \mathrm{~kb}$ on either sides of the damage. $\mathrm{H} 2 \mathrm{AX}$ not only marks the sites of stalled replication and DNA double strand breaks, but is also required for the recruitment of various proteins involved in DNA repair (Rogakou, Pilch et al. 1998, Ward and Chen 2001, Thiriet and Hayes 2005, Ewald, Sampath et al. 2007). 


\section{Checkpoint activation}

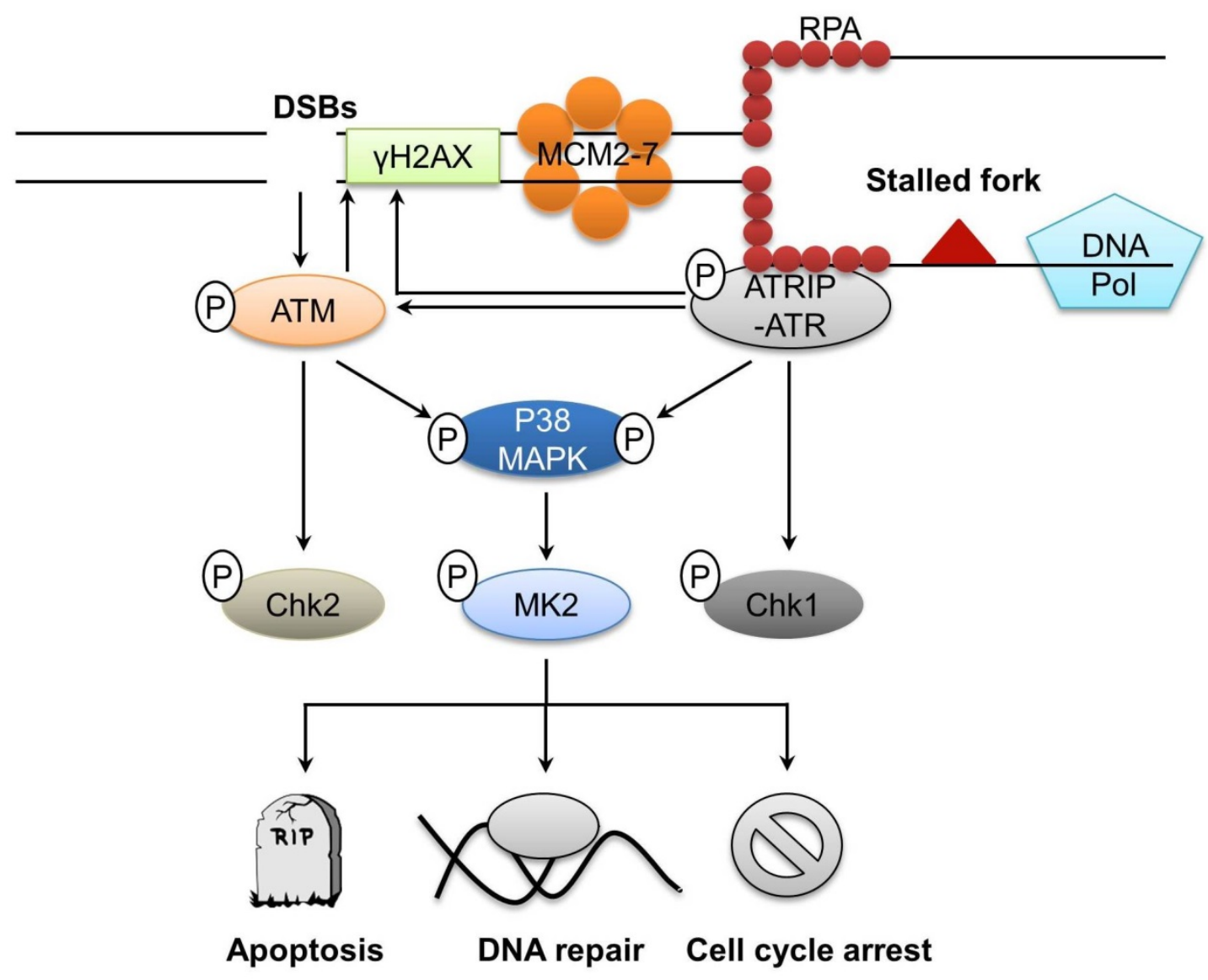

Figure 2.4 Activation of replication checkpoint signaling.

In the presence of an obstacle on the DNA template, DNA polymerase stalls. However, the MCM complexes continue to unwind the DNA and leave long stretches of SsDNA behind them. RPA coats these ssDNA which serves as a trigger for the checkpoint response. Various kinases including ATR (primarily), ATM and p38 are activated which phosphorylate and activate their downstream checkpoint kinases Chk1, Chk2 and MK2. These effector kinases bring about cell cycle arrest, DNA repair or apoptosis. ATM gets activated additionally by DNA DSBs. Not all phosphorylations are shown in this figure. See text for more details. Figure adapted with modifications from (Jones and Petermann 2012).

\subsubsection{Three's company: p38-MK2 joins the DDR network}

In addition to the canonical ATR-Chk1 and ATM-Chk2 signaling pathways, the p38MAPKMK2 pathway has recently been identified within the DDR network (Bulavin, Higashimoto et al. 2001,Manke, Nguyen et al. 2005, Reinhardt, Aslanian et al. 2007). In response to various stress conditions including hyperosmolar stress, TNF- $\alpha$ treatment and DNA damage; p38MAP kinase gets dually phosphorylated on the Thr 181 and Tyr 183 residues in the T-X$Y$ motif, by its upstream MAPKKs, namely, MKK3 and MKK6 (Brancho, Tanaka et al. 2003). Once activated, p38MAPK phosphorylates a number of downstream substrates including several transcription factors like p53, ATF2 etc., and kinases like MK2, MSK1 etc. However, 
one of the bona-fide substrates of p38MAPK is the kinase MAPKAPK2 alias MK2 (Mitogen Activated Protein Kinase Activated Protein Kinase 2), which gets activated when phosphorylated at Thr 334, and travels to the cytoplasm (Gaestel 2006). There MK2 phosphorylates a variety of substrates including the heat shock protein 27 (Hsp27) and the Cdc25 phosphatases. Thus, by inactivating Cdc25s, MK2 arrests the cell cycle (in the $S$ and $\mathrm{G} 2 / \mathrm{M}$ phases) in response to various DNA damaging agents. It is due to this nature of MK2 that it is also referred to as the 'checkpoint kinase 3' (Chk3) functioning parallel to Chk1 and Chk2 (Stokoe, Engel et al. 1992, Manke, Nguyen et al. 2005).

Hence, after activation, ATR, ATM and p38MAPK phosphorylate a plethora of downstream substrates which inhibit further DNA replication to arrest the cell cycle for DNA repair, or initiate apoptosis in case of overwhelming DNA damage. The following sections will give a detailed description the role played by some of these pathways in mediating the above mentioned effects.

\subsubsection{Inhibit globally but activate locally: Regulating replication initiation after stress}

\subsubsection{Checkpoint signaling inhibits unfired replication clusters}

Unlike their upstream activators which are restricted to the sites of DNA damage, Chk1 and Chk2 are highly mobile proteins which elicit a global response by relaying the signal from the nucleus to the entire cell. These Ser/Thr kinases phosphorylate the cell cycle regulatory dual specificity phosphatase, Cdc25A, and mark it for ubiquitin-mediated degradation. In the Xenopus system, this is further accompanied by a Chk1-mediated phosphorylation and activation of Wee1. Since Cdk2 activity requires an inactive Wee1 kinase and an active Cdc25A phosphatase, such an event results in the accumulation of a non-functional Cdk2, phosphorylated at Tyr 15 (Costanzo, Robertson et al. 2000, Falck, Mailand et al. 2001, Sorensen, Syljuasen et al. 2004). Along similar lines, in 2003, using Xenopus, Costanzo et al., reported the inactivation of $\mathrm{Cdc} 7$ kinase by ATR after topoisomerase inhibition (Costanzo, Shechter et al. 2003). Such a down regulation of Cdk2 and Cdc7 activity inhibits the helicase co-factor $\mathrm{Cdc} 45$, from loading onto origins, thus preventing origin firing from the 'unfired replicon clusters' during replicative stress (Shechter, Costanzo et al. 2004, Syljuasen, Sorensen et al. 2005, Jones and Petermann 2012). In this manner, a negative regulation on replication initiation protects the 'still-to-be-replicated' parts of genome from instability or damage until the replicative stress is relieved (Ge and Blow 2010, McIntosh and Blow 2012). This is accompanied by an increased origin firing within the replicon clusters which are already engaged in replication, to prevent any instability that might arise due to incomplete replication of these clusters. This phenomenon is explained in detail below. 


\subsubsection{Dormant origin firing in active replicon clusters}

Replication is bi-directional with each fork requiring a replisome (see 2.1.2). Thus, in principle, two MCM complexes should be enough for firing one origin. However, studies have revealed that MCM's are loaded in $\sim 20$ fold excess over the number of DNA bound ORC molecules (Hyrien, Marheineke et al. 2003). These excessive MCM complexes are distributed throughout the genome and license 'additional' sites on the DNA (origins) which have the potential to fire, but are kept dormant (inactive) by the mildly active ATR-Chk1 signaling in an unperturbed S-phase. Such a checkpoint regulation is required to ensure an optimal number of active replication forks at a given time. This is essential because excessive origin firing can deplete cells of the necessary replication factors and result in fork stalling or DNA DSBs (Marheineke and Hyrien 2004, Machida, Hamlin et al. 2005).
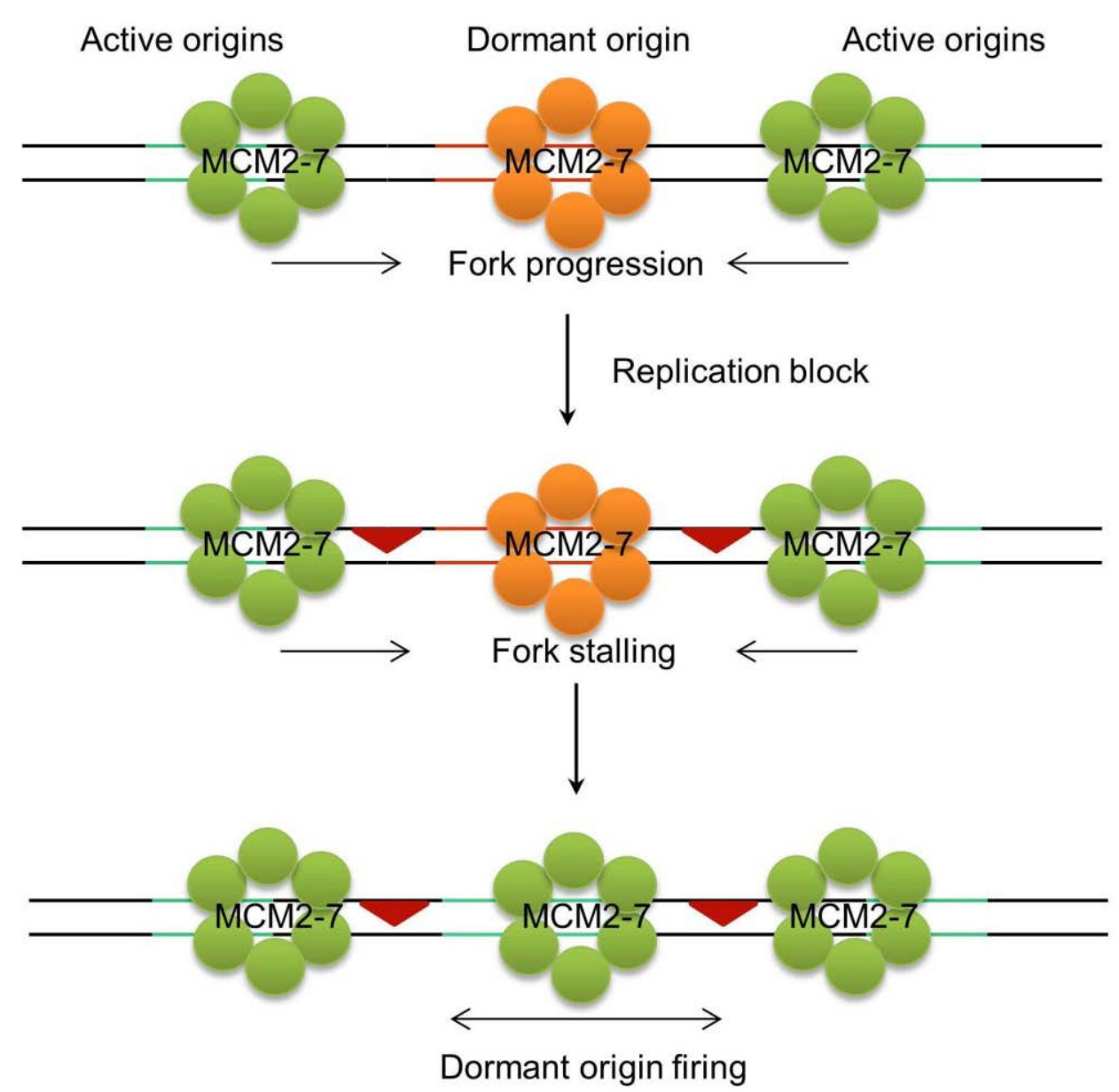

Figure 2.5 Dormant origin firing to promote replication under stress.

If two converging replication forks (shown with green MCMs, top) in an active replicon cluster, meet an obstacle on their way such that they are stalled (middle), the dormant origin in between them, now fires to complete the replication (lower). This is mediated by checkpoint signaling, which simultaneously also ensures that the blockade is removed. See text for more details. Figure adapted with modifications from (Blow, Ge et al. 2011). 
Under conditions of replicative stress, in active replicon clusters, two converging forks originating from adjacent origins may stall. In such a scenario, the 'dormant origins' present between them fire to complete replication (Woodward, Gohler et al. 2006, Ge, Jackson et al. 2007, Ibarra, Schwob et al. 2008, Blow, Ge et al. 2011) The mechanisms regulating dormant origin firing are not yet well elucidated. Even though it could be purely stochastic, recent studies have identified an ATR-PIk1 (Polo-like kinase-1) dependent phosphorylation of Orc2 (at Ser 188 in humans), to facilitate this process (Trenz, Errico et al. 2008, Song, Liu et al. 2011). Thus, checkpoint signaling works to regulate replication initiation in times of stress by inhibiting replication cluster activation but activating dormant origin firing near stalled forks.

\subsubsection{Mending the ends: Repair and restart of stalled replication forks}

Regulating replication in the manner as described above is required to arrest cell cycle for repairing the damaged after replicative stress. When a progressing replisome comes across a single strand gap or a bulky lesion on the parental DNA; cells make use of identical sister chromatids generated during DNA replication and employ the homologous recombination pathway (HR) to repair the damage (Saintigny, Delacôte et al. 2001). A key player involved in this pathway is Rad51, which after binding to the 3' single stranded overhangs or single stranded gaps, mediates homology search and promotes recombination into the homologous double stranded DNA (Sigurdsson, Van Komen et al. 2001). In case of DSBs generated at collapsed forks, HR needs the additional action of certain exonucleases like MRE11 (Meiotic Recombination 11) and EXO1 (Exonuclease 1) which assist the formation of 3' overhangs. (Nimonkar, Genschel et al. 2011). Such a recombination promotes the restart of a stalled fork which requires several accessory proteins like PARP1, BRCA2, BRCA1 and the FA complex. PARP1 (Poly (ADP-ribose) polymerase 1 ) is recruited to aberrant DNA structures and single strand gaps where it assists the loading of MRE11 to create the 3' overhangs, BRCA2

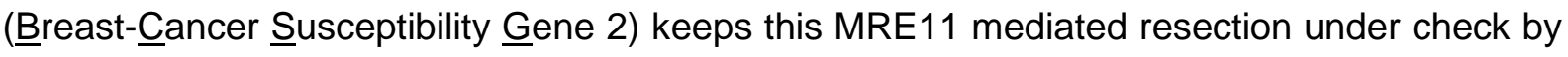
preventing excessive nuclease activity. These aforementioned proteins along with other adaptor molecules perform many key functions during DNA repair, whose details are beyond the scope of this thesis.

In addition to the above error-free repair pathway, an error-prone translesion synthesis (TLS) also exists which gives the cell an opportunity to switch its replicative DNA polymerase with a translesion polymerase (TLS polymerase). Such a polymerase is able to tolerate a variety of distortions in the DNA template and thus is able to replicate past these damages. The TLS polymerase switching requires the ubiquitin ligase Rad18 mediated mono-ubiquitination of PCNA as well (Hoege, Pfander et al. 2002, Prakash, Johnson et al. 2005, Jones and Petermann 2012). In addition to this, another level of regulation for TLS polymerases was shown in a recent study from Köpper et al., where the TLS polymerase activity was subjected 
to negative regulation by the checkpoint kinase - MK2, in response to gemcitabine induced DNA damage. This study established a role of MK2 in regulating DNA replication and repair, in addition to controlling cell cycle progression (Kopper, Bierwirth et al. 2013).

Apart from these specialized polymerases, cells also possess certain DNA helicases, namely BLM, WRN and FANCJ which exclusively unwind difficult-to-replicate secondary structures, thereby facilitating replisome progression. These helicases are also known to restart stalled forks by aiding HR. These processes are also thought to be dependent on the checkpoint, but their exact function in the same is still under investigation.

Even though the replication checkpoint acts to safeguard the genome, still, under conditions of extreme replicative stress, cell death pathways are initiated. This is to prevent the accumulation of DNA damage or aberrant DNA structures which could give rise to mutations in the next generation. How does a cell activate these pathways? What are the proteins involved? These questions will be addressed briefly in the next section.

\subsection{Cell death by apoptosis}

Under conditions of extreme stress, cells activate a programmed set of signaling events to undergo death - a phenomenon referred to as apoptosis. This cellular suicide is accompanied by various morphological changes, most notably; membrane blebbing, chromatin condensation and DNA fragmentation (Kerr, Wyllie et al. 1972).

Apoptosis can be triggered by both extrinsic and intrinsic stimuli. While extrinsic stimuli include death ligands (Fas-L or TNF- $\alpha$ ) binding to cell surface receptors, intrinsic stimuli encompass various conditions of cellular stress (DNA damage, heat shock etc.) that result in a compromised mitochondrial membrane potential. Irrespective of the stimuli, apoptosis requires a specific class of enzymes called caspases (cysteine aspartic proteases) that have cysteine in their active site and cleave their target proteins at aspartic residues. Owing to their proteolytic nature, these enzymes exist in an inactive zymogenic form (pro-caspases) in healthy cells and undergo activation only when cleaved (Nicholson 1999).

Caspases can be divided broadly into two categories based on their function: Initiator and Effector caspases. Initiator caspases are the upstream enzymes (Caspase 2, 8, 9, 10) that are brought together by certain adaptor proteins to form an aggregate. Such a complex formation stimulates the proteolytic activity of these enzymes, which subsequently cleave and activate their downstream targets - the effector caspases. The effector / executioner caspases (Caspase 3, 6, 7) chop down several cellular proteins. Some of the most important ones include: the nuclear lamins, whose cleavage leads to the breakdown of nuclear envelope; certain DNA repair proteins like PARP which get inactivated when cleaved; and 


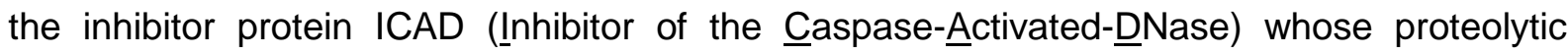
cleavage frees the DNase, CAD (Sakahira, Enari et al. 1998). The DNA fragmented by this DNase is packaged along with other cleavage products into small vesicles called apoptotic bodies, that are phagocytized by macrophages, and thus cleared from the surroundings. In this manner, apoptosis presents itself as a clean mechanism to get rid of damaged cells, avoiding a spillover of toxic contents into the surroundings. (Elmore 2007, Taylor, Cullen et al. 2008). Since replicative stress is an intrinsic trigger for apoptosis, in the next sections, a concise description of the various proteins involved in the mammalian intrinsic apoptotic pathway will be given (Rich, Allen et al. 2000).

\section{Do you have the potential? Bcl2 family proteins in regulating mitochondrial membrane integrity and apoptosis}

Mitochondria play a key role in activating the intrinsic pathway of apoptosis that involves mitochondrial outer membrane permeabilization mediated by the $\mathrm{Bcl} 2$ family proteins. This releases cytochrome $c$ from the inter-membrane space into the cytosol, where it interacts with APAF1 (Apoptotic Protease Activating Factor 1) to form a complex called the 'apoptosome'. Once assembled; the apoptosome recruits and activates the initiator procaspase 9, thus commencing a chain of caspase events culminating in the activation of caspase 3 and caspase 7 (Chinnaiyan 1999, Saelens, Festjens et al. 2004, Riedl and Salvesen 2007).

The mitochondrial Bcl2 (י-cell-lymphoma 2) family includes both pro-apoptotic and antiapoptotic proteins possessing $\mathrm{BH}$ domain (ㅡㅡㄴ 2 Homology domain). The anti-apoptotic family includes 5 members, namely Bcl-2-related gene A1 (A1), Bcl-2, Bcl-2-related gene long isoform (BCL-XL), BCL-w, and myeloid cell leukemia 1 (Mcl-1). Each of these anti apoptotic proteins contains four $\mathrm{BH}$ domains that mediate their interaction with their pro-apoptotic counterparts (Green and Evan 2002). This keeps all the pro-apoptotic proteins inactive until an appropriate signal is received. For example; Mcl-1 interacts with Bak, one of the effector pro-apoptotic protein, and keeps it in an inactive monomeric form under healthy conditions (Willis, Chen et al. 2005).

On the other hand, the pro-apoptotic family is further sub-classified into $\mathrm{BH} 3$ only and effector proteins. The effector proteins include Bak and Bax (each having three $\mathrm{BH}$ domains) that homo-oligomerize and form pores in the outer mitochondrial membrane (OMM), thus disturbing $\mathrm{OMM}$ integrity and leading to mitochondrial outer membrane permeabilization (MOMP). The BH3 only proteins (Puma, Noxa, Bad, Bim etc.) have one $\mathrm{BH}$ domain ( $\mathrm{BH} 3)$, and act either as 'activators', 'de-repressors' or 'sensitizers', depending on their interaction with the various members of the Bcl-2 family. While 'activators' like Bid and Bim directly 
interact with Bax/Bak and induce their oligomerization, promoting MOMP; de-repressors and sensitizers regulate the activity of anti-apoptotic proteins by inhibiting them. De-repressors and sensitizers act in the following manner: Anti-apoptotic proteins (e.g. Bcl2) remain bound to the direct activators (e.g. Bim) repressing their function under normal conditions. However, during cellular stress like DNA damage, the de-repressor BH3 family protein (e.g. Puma) gets induced and now competes with the direct activator for binding to the anti-apoptotic

protein. In this manner, the direct activator is released and the anti-apoptotic protein is inhibited by $\mathrm{BH} 3$ only de-repressor. On the contrary, 'sensitizers' remain bound to the antiapoptotic proteins under normal and stressful conditions, thus keeping the direct activators uninhibited and free to promote MOMP. The same protein can function as a de-repressor or a sensitizer depending on the stimuli. In this manner, regulating the interaction among $\mathrm{Bcl} 2$ family proteins is crucial for determining the survival outcome of a cell during various stress conditions including replicative stress (Chipuk, Moldoveanu et al. 2010).

However, if a damaged cell evades apoptosis, it can accumulate genetic mutations that give it an uncontrolled capacity to proliferate, thereby leading to cancer development. The following section will shed some light on this.

\subsection{Cancer and chemotherapy: DNA replication as a double edged sword}

DNA replication is the basis of all life; the deregulation of which can generate mutations that result in uncontrolled cellular proliferation and thus tumor formation. According to the model presented by Hanahan and Weinberg in 2000 , tumor cells need to acquire certain hallmarks to become cancerous. These are summarized as follows: sustained proliferative signaling, ability to evade growth suppressors, replicative immortality, potential to invade surrounding tissues and metastasize, capacity to induce angiogenesis and resist cell death (Hanahan and Weinberg 2000). In 2011, this picture was enlarged by the addition of certain emerging characteristics like the ability of cancer cells to evade the immune system and deregulate cellular metabolism (Hanahan and Weinberg 2011). Thus, cancer is a 'disease of self', where genetic changes caused by exogenous or endogenous agents are responsible for its manifestation. This has motivated a large part of the scientific community to focus its research on understanding the basic biology of cancer and develop drugs that could help treat it.

Chemotherapy is one of the many lines of cancer treatment which uses chemical substances to kill rapidly proliferating cells. Based on their mode of action and chemical structure, chemotherapeutic agents are classified into various categories some of which include: microtubule and topoisomerase inhibitors, alkylating agents, antimetabolites, antifolates and 
cytotoxic antibiotics (Malhotra and Perry 2003). Among these, some selected agents that act by inhibiting DNA replication and generating DNA damage are outlined below.

\section{Antimetabolites and Antifolates}

Antimetabolites and antifolates are drugs that directly inhibit DNA synthesis, albeit with different mechanisms of action. Antifolates like methotrexate inhibit the enzyme dihydrofolate reductase (DHFR), which is required for the production of folate coenzymes. Since these folates assist purine and pyrimidine biosynthesis, such a drug is able to inhibit the synthesis of DNA by disturbing the nucleoside pool balance. On the other hand, antimetabolites like gemcitabine (2', 2'-Difluoro-desoxycytidine) and cytarabine (1- $\beta$-D-Arabinofuranosylcytosine) mimic the naturally occurring nucleosides (in this case cytosine) and are falsely incorporated into the replicating DNA instead of its normal counterpart. In addition, some antimetabolites like gemcitabine are also able to inactivate the enzyme ribonucleotide reductase, thus, enhancing its ability to interfere with replication by depleting the cellular pools of dNTPS. These are also called S-phase specific drugs as they target only the cells which are actively engaged in duplicating their genome (Ewald, Sampath et al. 2008).

\section{Alkylating agents and platinum based drugs}

DNA can also be damaged by covalently linking an alkyl group to one of its bases, by agents like mechlorethamine, cyclophosphamide etc. or by crosslinking the bases with each other (inter-strand or intra-strand crosslink) using platinum-based drugs like cisplatin, carboplatin etc. Unlike the antimetabolites, these drugs act in all the phases of cell cycle (McClean, Costelloe et al. 1999, Kelland 2007).

\section{Topoisomerase inhibitors}

Topoisomerases are the enzymes required for relaxing negative and positive supercoils that are created ahead of the unwound DNA. Drugs targeting these enzymes can inhibit both DNA replication and transcription, and also induce DSBs. This is achieved either via blocking the activity of the enzyme, or increasing its levels on DNA, thereby creating an obstruction for the progressing fork. Examples include camptothecin, etoposide etc.

Besides these, numerous other approaches are taken for treating cancer. These include surgical removal of the tumor, radiation therapy, chemotherapy using microtubule inhibitors, proteasome inhibitors and anthracyclines; hormonal and immunotherapy. 


\subsection{Chemoresistance and combination therapy}

Despite the presence of various chemotherapeutic agents, achieving effective treatment has become difficult due to drug resistance. This loss of sensitivity can be acquired during therapy or be intrinsically present before the start of a treatment regime. The most common reasons for chemoresistance include effective drug efflux, increased drug inactivation, efficient DNA repair mechanisms and increased ability to evade apoptosis. Whether intrinsic or acquired, in both cases, resistant cells often become insensitive to other lines of chemotherapy as well (Wilson, Longley et al. 2006). This raises the need for developing combination therapies that might help in chemosensitization. One such chemotherapy based combination regime makes use of small molecules to inhibit the proliferative MAPK signaling pathway, which is often deregulated in various cancers.

MAPKs or mitogen activated protein kinases are Ser/Thr kinases that when activated, govern the cellular outcome to a variety of external stimuli including growth factors, cytokines and stress signals. The three major branches of MAPK signaling include - ERK (Extracellular signal Related Kinase), p38MAPK, and JNK (c-jun-N-terminal Kinase). ERK responds to growth signals and promotes proliferation; whereas p38MAPK and JNK are majorly activated during cellular stress and facilitate survival or apoptosis depending on the stimuli (Johnson and Lapadat 2002). Among these, the ERK signaling pathway has garnered a lot of attention from researchers due to its oncogenic potential, which has led to the development of several inhibitors against the EGFR-Raf-MEK-ERK pathway (Roberts and Der 2007). This further makes it an interesting target in combination therapies as well. A well-known example of such an inhibitor used in gemcitabine combination therapy is an EGFR inhibitor, erlotinib, which is FDA approved for the treatment of metastatic pancreatic cancer (http://www.cancer.gov/cancertopics/druginfo/fda-erlotinib-hydrochloride\#Anchor-Pancreati-

44285) (Moore, Goldstein et al. 2007). Besides this, several other drugs like sofarenib (against EGFR), imatinib (Against Bcr-abl tyrosine kinase) are also been approved. Still many more are in the different stages of clinical trials.

\subsection{Mitogen activated protein kinase phosphatases (MKPs)}

Since MAPKs control cellular proliferation and stress response, monitoring their activity is extremely important to avoid any deregulation that might assist tumor formation. This regulation is made possible by phosphatases, which reverse the phosphorylation required for MAPK activity. In response to an appropriate stimulus, MAPK gets dually phosphorylated on both Thr and Tyr residues of its T-X-Y motif. This phosphorylation and activation is mediated by its upstream MAPK Kinase (MKK), which is in turn activated by MAPK Kinase Kinase (MKKK). However, this signal is subjected to negative regulation by MAPK phosphatases 
(MKP). These enzymes belong to the family of dual specificity phosphatases, and are able to bind and dephosphorylate MAPKs on both the Thr and Tyr residues, thereby inhibiting the respective pathway (Dhillon, Hagan et al. 2007, Wu 2007). This makes the role of MKPs equally paramount not only in cancer development and progression, but also in chemotherapeutic response.

The MKP family has eleven members, which share structural homology with each other. All of them possess a non-catalytic N-terminal domain which share homology with the Cdc25 phosphatases; and a C-terminal catalytic domain that displays a sequence homology related to the $\mathrm{VH}-1$ phosphatase encoded by the vaccinia virus. Inspite of this structural similarity, MKPs are further sub-classified into three categories - Type I, Type II and Type III; depending on their substrate specificity and sub-cellular localization. The section below will focus on one of these MKPs, named MKP1 (Bermudez, Pages et al. 2010).

\subsubsection{Switching off the MAPK signaling by mitogen activated protein kinase phosphatase 1 (MKP1)}

MKP1 is the archetypal member of the MKP family. Originally discovered as a growth inducible nuclear phosphatase, MKP1 was later shown to be induced in response to a variety of stress stimuli including oxidative stress, heat shock and DNA damage (Keyse and Emslie 1992, Alessi, Smythe et al. 1993, Liu, Gorospe et al. 1995). Using its N-terminal kinase interaction motif, MKP1 binds to its substrate and undergoes a conformational change that enhances its catalytic activity. Both in-vitro and cell culture approaches have identified p38MAPK and JNK (also called the stress activated protein kinases or SAPKs) as the preferential substrates of MKP1 (Slack, Seternes et al. 2001, Owens and Keyse 2007).

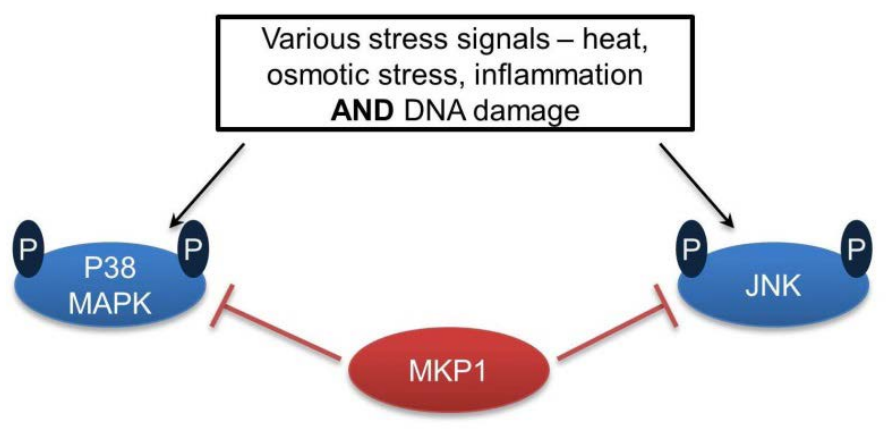

Figure 2.6 A schematic depicting the role of MKP1.

Upon receiving any kind of stress stimulus, like heat, osmotic stress, or even DNA damage, cellular stress response pathways are activated which include the p38MAPKs and JNKs. These kinases promote cell cycle arrest and apoptosis to combat the stress. However, a negative regulator of these kinases, a phosphatase MKP1 (alias DUSP1) is also activated which tries to balance this activating signal by dephosphorylating the p38MAPK and JNK on their T-X-Y motif. See text for more details. 
Activated by its upstream kinase MKK4 and MKK7, JNK is known to regulate cell cycle progression and apoptosis in response to various stress stimuli, by phosphorylating a number of downstream targets including c-Jun (an active part of the transcription factor AP1), p53, and certain members of the Bcl2 family. A similar role is played by p38MAPK, which has been described in section 2.4.2. However, the effects of these kinases are largely dependent on the strength and kind of stimulus. A stronger stress stimulus leads to cell death while a milder one results in cell cycle arrest. (Davis 2000, Wagner and Nebreda 2009).

Due to its ability to negatively regulate these MAPKs, the last decade has revealed an important role of MKP1 in cancer development, progression and chemoresistance.

\subsubsection{MKP1 in cancer and chemoresistance}

MKP1 has been extensively studied in human tumors, where an alteration of its expression pattern is observed. This is shown to be largely dependent on the grade and type of cancer. Breast, non-small cell lung and pancreatic carcinomas showed an increased expression of MKP1, while ovarian carcinoma showed a down-regulation of this protein. Furthermore, a fluctuation of MKP1 expression is observed within one type of cancer, with the lower grade carcinomas up-regulating MKP1 and the higher grade carcinomas showing a decrease in the expression. This kind of situation is most frequently observed in colon, prostate, bladder and liver carcinomas. Even though it is seemingly difficult to understand such an inconsistent expression pattern of MKP1, it is speculated that having lower levels of MKP1 facilitates proliferation by hyper-activating the ERK pathway, while an up-regulation is required to shut off the apoptotic SAPK pathways (Wu 2007, Boutros, Chevet et al. 2008). While these studies establish MKP1 as an important player in cancer development and progression, overexpression of MKP1 has also been correlated with chemoresistance. In 1998, Franklin and co-workers reported a protective role for MKP1 in human leukemic cells against UV induced apoptosis (Franklin, Srikanth et al. 1998). Since then, many studies have been performed using various genotoxic agents, to evaluate MKP1 in mediating drug resistance. While an

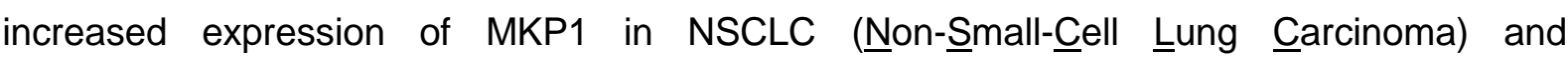
osteosarcoma was responsible for cisplatin resistance due to an attenuated JNK signaling; MKP1-deficient MEFs were sensitized to anisomycin treatment, due to an active p38MAPK pathway (Wu and Bennett 2005, Chattopadhyay, Machado-Pinilla et al. 2006, Wang, Zhou et al. 2008). Furthermore, in breast cancers, MKP1 over-expression was shown to reduce the cytotoxic effects of doxorubicin, microtubule inhibitors and alkylating agents; by targeting the JNK pathway (Small, Shi et al. 2007). Taken together, these studies establish a key role of MKP1 in chemoresistance, making it an attractive candidate to research. 


\subsection{Scope of thesis}

As mentioned in the previous section, MKP1 plays a protective role in response to various phase unspecific DNA damaging agents, thus determining the outcome of these chemotherapeutic regimens. This nature of MKP1 motivated us to explore its function, specifically during conditions of replicative stress. Furthermore, as various checkpoint kinases remain active even during an unperturbed cell cycle to ensure efficient and faithful replication, we were keen to inspect the contribution of this phosphatase in unstressed conditions as well. These studies will add to our existing knowledge about phosphatases, particularly in the regulation of checkpoint signaling under replicative stress and normal conditions.

In addition to the basic translational research, these investigations will also help to examine the relevance of MKP1 as a potential drug target to increase the sensitivity of cancer cells to S-phase targeting drugs. As reported by Köpper et al., the kinase MK2 of the p38/MK2 pathway when inhibited protects cells from gemcitabine induced DNA damage. Thus, an activation of this kinase might sensitize tumor cells towards chemotherapy like gemcitabine. This makes it more interesting for us to study MKP1 and use inhibitors against it that might help increase the activity of p38/MK2 pathway and perhaps also lead to chemosensitization.

With these ideas in mind, experiments were conducted to monitor checkpoint activation, replication dynamics and cellular survival, using a pharmacological inhibitor of MKP1, termed $\mathrm{BCl}$, and a replicative stress agent, gemcitabine. We employed immunoblot assays to look for the activation of several DNA damage response and cellular survival/apoptosis markers. Furthermore, DNA fiber assay was the technique used for studying DNA replication kinetics. 


\section{Materials}

\subsection{Technical Devices}

Blotting chamber

Cell counting chamber Neubauer improved

Centrifuge $5415 R$

Centrifuge 5810R

Centrifuge Megafuge 1.0R

Chemiluminescence imager Chemocam HR 163200

Electrophoresis system, for SDS-PAGE

Foil swelding machine Vacupack plus

Freezer $-20^{\circ} \mathrm{C}$

Freezer $-80^{\circ} \mathrm{C}$

Heating Block

Heating Block HLC

Ice-machine $B 100$

Incubator for bacteria

Incubator for bacteria Minitron

Incubator for cell culture Hera Cell 150

Laminar flow cabinet Hera Safe

Liquid nitrogen tank LS 4800

Magnetic stirrer MR Hei-Standard

Magnetic stirrer MR3001

Microscope Axovert 40C

Microscope, Axiocam MRC Scope A1

Mini Centrifuge MCF-2360

PCR machine for qPCR CFX96, C1000

PCR machine Termocycler T personal

Personal computer

pH-meter WTW-720

Pipet Multipette

Pipet, electric Portable-XP

Pipets Eppendorf Research Series 2100

(0.1-2.5 $\mu \mathrm{L} ; 0.5-10 \mu \mathrm{L} ; 10-100 \mu \mathrm{L} ; 100-$

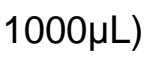

Pipette, multichannel Research Plus
Biozym, Hessisch Oldendorf, Germany

Brand, Wertheim, Germany

Eppendorf, Hamburg, Germany

Eppendorf

Heraeus, Thermo Scientific, Waltham, MA, United States

Intas Science Imaging Instruments, Göttingen, Germany

Amersham Biosciences, GE Healthcare, Little Chalfont, United Kingdom

Krups, Groupe SEB, Lyon, France

Liebherr, Bulle, Switzerland

Heraeus, Thermo Scientific

Grant Instruments, Hillsborough, NJ, United States

HLC Biotech, Ditabis, Pforzheim, Germany

Ziegra, Isernhagen, Germany

Memmert, Schwabach, Germany

Infors HT, Basel, Switzerland

Heraeus, Thermo Scientific, Waltham, MA, United States

Heraeus, Thermo Scientific

Taylor-Wharton, Theodore, AL, United

States

Heidolph, Schwabach, Germany

Heidolph

Zeiss, Oberkochen, Germany

Zeiss

LMS, Tokyo, Japan

Bio-Rad Laboratories

Biometra, Göttingen, Germany

Dell, Round Rock, TX, United States

WTW, Weilheim, Germany

Eppendorf

Drummond, Broomal, PA, United States

Eppendorf

Eppendorf 
Power supply unit Powerpack P25T

Refrigerator $4^{\circ} \mathrm{C}$

Roller RM5 V-30

Rotator PTR 300

Scales Acculab ALC-6100.1

Scales LE623S

Scanner CanoScan 8600F

Sequencer, automated $A B I 3100$

Shaker PROMAX 2020

Sonication device Bioruptor

Spectrophotometer NanoDrop ND-1000

Thermomixer comfort

Timer

Vacuum pump

Vortex Genie 2

Water bath TW 20

\subsection{Consumables}

96-well plates for qPCR

Bacteria culture dishes

Bacteria culture vials $(14 \mathrm{~cm})$

Cell culture dishes $(10 \mathrm{~cm}, 15 \mathrm{~cm})$

Cell culture plates (6-well)

Cell scraper $(16 \mathrm{~cm}, 25 \mathrm{~cm})$

Cover slips

Cryo tubes Cryoline

Filter tips $(10 \mu \mathrm{L})$

Filter tips $(20 \mu \mathrm{L}, 200 \mu \mathrm{L}, 1,000 \mu \mathrm{L})$

Glass Slides Superfrost

Parafilm

Pipet tips (10 $\mu \mathrm{L}, 20-200 \mu \mathrm{L}, 1,000 \mu \mathrm{L})$

Protran nitrocellulose transfer membrane

PVDF membrane Amersham Hybond-P

Reaction tube $(0.2 \mathrm{~mL})$

Reaction tube $(0.5 \mathrm{~mL}, 1.5 \mathrm{~mL}, 2.0 \mathrm{~mL}$ )

Reaction tube $(15 \mathrm{~mL}, 50 \mathrm{~mL})$

Safe-lock reaction tube $(1.5 \mathrm{~mL})$

Sterile filter

Syringe
Biometra

Liebherr

CAT, Staufen, Germany

Grant Instruments

Sartorius, Göttingen, Germany

Sartorius

Canon, Tokyo, Japan

Applied Biosystems, Life Technologies

Heidolph

Diagenode, Liège, Belgium

PeqLab, Erlangen, Germany

Eppendorf

Oregon Scientific, Portland, OR, United

States

IBS Integra Biosciences, Fernwald,

Germany

Scientific Industries, Bohemia, NY, United

States

Julabo Labortechnik, Seelbach, Germany 4titude, Wotton, United Kingdom

Sarstedt, Nümbrecht, Germany

Becton Dickinson

Greiner, Frickenhausen, Germany

Greiner

Sarstedt

Menzel, Thermo Scientific

Nunc, Thermo Scientific

Starlab, Hamburg, Germany

Sarstedt

Menzel, Thermo Scientific

Brand

Greiner

Whatman, Dassel, Germany

GE Healthcare, Life Sciences

Sarstedt

Eppendorf

Greiner

Eppendorf

Millipore, Merck

Henke-Sass, Wolf, Tuttlingen, Germany 
Syringe canula (different sizes)

Transparent sealing foil for 96-well plate

Whatman paper

\subsection{Chemicals and reagents}

Acetic acid

Agar

Albumin Fraction V (Bovine Serum Albumine, BSA)

Ammonium persulfate (APS)

Ammonium sulfate $\left(\left(\mathrm{NH}_{4}\right)_{2} \mathrm{SO}_{4}\right)$

Ampicillin

Bromophenol blue

Calcium chloride dihydrate $\left(\mathrm{CaCl}_{2} \times 2 \mathrm{H}_{2} \mathrm{O}\right)$

Chlorodeoxyuridine (CldU)

Chloroform

Complete Mini Protease Inhibitor

Dimethyl sulfoxide (DMSO)

Dithiotreitol (DTT)

deoxynucleotide triphosphates (dNTPs)

Ethanol 99.8\%

Ethanol $99.9 \%$ p.a. (EtOH)

Ethylene diamine tetraacetatic acid (EDTA)

Formaldehyde, $37 \%$ solution

Glycerol

Glycine

Glycogen blue

HEPES

Hydrogen chloride $(\mathrm{HCl})$

Immersion oil

lododeoxyuridine (IdU)

Isopropanol

Lipofectamine 2000

Magnesium chloride $\left(\mathrm{MgCl}_{2}\right)$ for PCR

Magnesium chloride hexahydrate $\left(\mathrm{MgCl}_{2} \mathrm{X}\right.$

$6 \mathrm{H}_{2} \mathrm{O}$ )

Methanol >99\% (MetOH)

Nailpolish

Nonidet P-40 substitute (NP-40)

Nuclease free water

Peptone
B.Braun, Melsungen, Germany

4titude

Whatman

Roth, Karlsruhe, Germany

Sigma-Aldrich, St. Louis, MO, United

States

Roth

Roth

Roth

AppliChem, Darmstadt, Germany

Sigma-Aldrich

Roth

Sigma-Aldrich

Roth

Roche, Basel, Schweiz

AppliChem

Sigma-Aldrich

Bio-Budget, Krefeld, Germany

Roth

Merck

Roth

Roth

Roth

Roth

Ambion, Life Technologies

Roth

Roth

Zeiss

Sigma-Aldrich

Th. Geyer, Renningen, Germany Invitrogen, Life Technologies

Fermentas, Thermo Scientific

Roth

Roth

Sigma Aldrich

Ambion, Life Technologies

Roth 
Ponceau S

Potassium chloride $(\mathrm{KCl})$

Potassium hydrogenphosphate $\left(\mathrm{KH}_{2} \mathrm{PO}_{4}\right)$

Prestained Protein Ladder

Protein-G-Sepharose (PGS)

RNase inhibitor

Rotiphorese Gel 30

Sodium acetate (NaAc)

Sodium bicarbonate $\left(\mathrm{NaHCO}_{3}\right)$

Sodium chloride $(\mathrm{NaCl})$

Sodium deoxycholate

Sodium dodecyl sulfate (SDS)

Sodium hydrogenphosphate heptahydrate

$\left(\mathrm{Na}_{2} \mathrm{HPO}_{4} \times 7 \mathrm{H}_{2} \mathrm{O}\right)$

Sodium hydroxide $(\mathrm{NaOH})$

Sodium orthovanadate

Sodium pyrophosphate decahydrate

SYBR green

Tetracycline

Tetramethylethylenediamine (TEMED)

Trasylol

Trehalose

Trisamine (Tris)

Triton X-100

Trizol

Tween 20

Vectashield mounting medium

Yeast extract

$\beta$-Mercaptoethanol
Roth

Roth

Roth

Fermentas, Thermo Scientific

GE Healthcare

Fermentas, Thermo Scientific

Roth

Roth

Roth

Roth

Applichem

Roth

Roth

Sigma-Aldrich

Sigma-Aldrich

Apllichem

Invitrogen, Life Technologies

Sigma-Aldrich

Roth

Bayer, Leverkusen, Germany

Sigma-Aldrich

Roth

Applichem

Invitrogen, Life Technologies

Applichem

Vector Laboratories, Burlingame, CA,

United States

Sigma-Aldrich

Roth

\subsection{Buffers and solutions}

Cell lysis buffer

Urea $2.5 \mathrm{M}$

RIPA lysis buffer

$100 \%$

for SDS PAGE, diluted with 6x laemmli 1:5

\section{ColP buffer}

\begin{tabular}{lr}
\hline Tris, $\mathrm{pH} 7.5$ & $50 \mathrm{mM}$ \\
$\mathrm{NaCl}$ & $150 \mathrm{mM}$ \\
$\mathrm{NP}-40$ & $0.20 \%$ \\
$\mathrm{Na}$ deoxycholate & $0.25 \%$
\end{tabular}


Protease inhibitors

dissolved in $\mathrm{H}_{2} \mathrm{O}$

Fiber assay blocking solution

$\begin{array}{ll}\text { BSA } & 3.0 \% \\ \text { Tween } 20 & 0.1 \%\end{array}$

dissolved in PBS

Fiber assay fixative

MetOH 75\%

Acetic Acid 25\%

Fiber assay spreading buffer

\begin{tabular}{lr}
\hline Tris, $\mathrm{pH} 7.4$ & $200 \mathrm{mM}$ \\
EDTA & $50 \mathrm{mM}$ \\
SDS & $0.5 \%$
\end{tabular}

dissolved in $\mathrm{H}_{2} \mathrm{O}$

Laemmli buffer, $6 x$

\begin{tabular}{lr}
\hline Tris pH 6.8 & $0.35 \mathrm{M}$ \\
Glycerin & $30.00 \%$ \\
SDS & $10.00 \%$ \\
Dithiotreitol & $9.30 \%$ \\
Bromophenol blue & $0.02 \%$
\end{tabular}

dissolved in $\mathrm{H}_{2} \mathrm{O}$

Phophate buffered saline (PBS), pH 7.5

$\mathrm{NaCl} 24.00 \mathrm{mM}$

$\mathrm{KCl} \quad 0.27 \mathrm{mM}$

$\mathrm{Na}_{2} \mathrm{HPO}_{4} \times 7 \mathrm{H}_{2} \mathrm{O} \quad 0.81 \mathrm{mM}$

$\mathrm{KH}_{2} \mathrm{PO}_{4} \quad 0.15 \mathrm{mM}$

dissolved in $\mathrm{H}_{2} \mathrm{O}$

Ponceau S solution

Ponceau S 0.5\%

Acetic acid $\quad 1.0 \%$

dissolved in $\mathrm{H}_{2} \mathrm{O}$

qPCR reaction buffer, 10x

\begin{tabular}{lr}
\hline Tris, $\mathrm{pH} \mathrm{8,8}$ & $750 \mathrm{mM}$ \\
$\left(\mathrm{NH}_{4}\right)_{2} \mathrm{SO}_{4}$ & $200 \mathrm{mM}$ \\
Tween 20 & $0.1 \%$ \\
dissolved in $\mathrm{H}_{2} \mathrm{O}$ &
\end{tabular}


qPCR reaction mix, 25x

10x qPCR reaction 1x

buffer

SybrGreen

1:80,000

$\mathrm{MgCl}_{2}$

$3.0 \mathrm{mM}$

Trehalose in $10 \mathrm{mM}$

$300.0 \mathrm{mM}$

Tris, pH 8,5

dNTPs

$0.2 \mathrm{mM}$

Triton X-100

$0.25 \%$

Taq polymerase

$20 \mathrm{U} / \mathrm{mL}$

dissolved in $\mathrm{H}_{2} \mathrm{O}$

RIPA lysis buffer, pH 7.5

\begin{tabular}{lr}
\hline Triton X-100 & $1.0 \%$ \\
Na desoxycholate & $1.0 \%$ \\
SDS & $0.1 \%$ \\
$\mathrm{NaCl}$ & $150 \mathrm{mM}$ \\
EDTA & $10 \mathrm{mM}$ \\
Tris, pH 7.5 & $20 \mathrm{mM}$ \\
Trasylol & $50,000 \mathrm{KIU}^{\star}$ \\
dissolved in $\mathrm{H}_{2} \mathrm{O}$ &
\end{tabular}

${ }^{*}$ KIU: Kallikrein Inactivator Units

SDS running buffer

\begin{tabular}{lr}
\hline Tris & $25.0 \mathrm{mM}$ \\
Glycin & $86.1 \mathrm{mM}$ \\
SDS & $3.5 \mathrm{mM}$
\end{tabular}

dissolved in $\mathrm{H}_{2} \mathrm{O}$

Tris buffered saline + Tween 20 (TBST), pH 7.6

\begin{tabular}{lr}
\hline Tris & $50 \mathrm{mM}$ \\
$\mathrm{NaCl}$ & $150 \mathrm{mM}$ \\
Tween 20 & $0.1 \%$ \\
dissolved in $\mathrm{H}_{2} \mathrm{O}$ &
\end{tabular}

Western blot blocking solution

BSA $5 \%$

dissolved in TBST

Western blot buffer, pH 8.3

\begin{tabular}{lr}
\hline Tris & $25 \mathrm{mM}$ \\
Glycin & $192 \mathrm{mM}$
\end{tabular}


MetOH

$20 \%$

dissolved in $\mathrm{H}_{2} \mathrm{O}$

\subsection{Chemotherapeutics}

\begin{tabular}{lll} 
Name & Systematic name & Company \\
\hline Gemcitabine & $2^{\prime}, 2^{\prime}$-difluorodeoxycytidine $(\mathrm{dFdC})$ & Eli Lilly, Indianapolis, IN, \\
& & United States
\end{tabular}

\subsection{Inhibitors}

\begin{tabular}{llll} 
Name & Commercial name & Target & Company \\
\hline ATM Inh & KU55933 & ATM & Selleckchem \\
Caspase Inh & Z-VAD-FMK & Caspases & Enzo Life Sciences \\
MK2 Inh & MK2 III & MK2 & Calbiochem, Merck \\
MKP1 Inh & BCl.HCl & MKP1 & Sigma-Aldrich
\end{tabular}

\subsection{Enzymes and buffers}

\section{Reagent}

Buffer for M-MuLV RT, 10x

Buffer for Taq with $\mathrm{KCl}, 10 \mathrm{x}$

M-MuLV Reverse transcriptase (RT)

Taq DNA polymerase (Taq)

\section{Company}

New England Biolabs, Ipswich, MA, United States

Fermentas, Thermo Scientific

New England Biolabs

Fermentas, Thermo Scientific

\subsection{Kits}

Name

BigDye Terminator v3.1 Cycle Sequencing kit Immobilon Western HRP Substrate Peroxide

Solution

Invisorb Spion Plasmid Mini Kit Two

PureYield Plasmid Midiprep System

SuperSignal West Femto Maximum Sensitivity Thermo Scientific

Substrate

\section{9 small interfering RNAs}

\begin{tabular}{ll} 
Name (identifies target) & Sequence \\
\hline Negative Control No. 2 & undisclosed \\
MKP1 & sense: 5'-CCACCACCGUGUUCAACUUtt-3' \\
& antisense: 5'-AAGUUGAACACGGUGGUGGtg-3'
\end{tabular}

All siRNAs are Silencer Select from Ambion, Life Technologies. 


\subsection{Primers}

\begin{tabular}{lll} 
Name & Sequence & Application \\
\hline BGH reverse & 5'-TAGAAGGCACAGTCGAGG-3' & sequencing \\
CMV forward & 5'-CGCAAATGGGCGGTAGGCGTG-3' & sequencing \\
XL39 reverse & 5'ATTAGGACAAGGCTGGTGGG-3' & sequencing \\
VP1.5 forward & 5'-GGACTTTCCAAAATGTCG-3' & sequencing \\
anchored oligo-dT & dT'23VN & RT-PCR \\
random nonamer & 5'-NNNNNNNNN-3' & RT-PCR \\
Cdc7 forward & 5'-AGATTGAGGACAAAATTGGAGAA-3' & qPCR \\
Cdc7 reverse & 5'-CAGCCACTGTTAGGCACTGA -3' & \\
GAPDH forward & 5'- & \\
& TGAAGGTCGGAGTCAACGGATTTGGT- & \\
GAPDH reverse & 5'-GCAGAGATGATGACCCTTTTGGCTC- & \\
& 3' &
\end{tabular}

\subsection{Plasmids}

\begin{tabular}{|c|c|c|}
\hline Name & Source & Description \\
\hline pcDNA3.1 & $\begin{array}{l}\text { Invitrogen, Life } \\
\text { Technologies }\end{array}$ & $\begin{array}{l}\text { Expression vector for the } \\
\text { exogenous expression of proteins } \\
\text { under the control of a CMV } \\
\text { promoter in eukaryotic cells; } \\
\text { ampicillin resistance. See Fig. } 7.6 \\
\text { for vector map. }\end{array}$ \\
\hline $\begin{array}{l}\text { pcDNA3.1-Mcl-1-V5-His- } \\
\text { TOPO }\end{array}$ & $\begin{array}{l}\text { Addgene Plasmid } 25375 \text {, } \\
\text { gift from Roger Davis }\end{array}$ & $\begin{array}{l}\text { pcDNA3 vector with open reading } \\
\text { frame coding for human } \mathrm{Mcl}-1 \\
\text { inserted in the MCS. }\end{array}$ \\
\hline pCMV6-Myc-DDK-DUSP1 & $\begin{array}{l}\text { Origene, RC205220, } \\
\text { NM_004417 }\end{array}$ & $\begin{array}{l}\text { pCMV6-Entry vector with open } \\
\text { reading frame for human DUSP1 } \\
\text { (alias MKP1) inserted in MCS. See } \\
\text { Fig. } 7.6 \text { for vector map. }\end{array}$ \\
\hline pCMV6-Flag-Mcl-1 & $\begin{array}{l}\text { Addgene Plasmid } 25392, \\
\text { gift from Roger Davis }\end{array}$ & $\begin{array}{l}\text { pcDNA3 vector with open reading } \\
\text { frame coding for human } \mathrm{Mcl}-1 \\
\text { inserted in the MCS. }\end{array}$ \\
\hline
\end{tabular}




\subsection{Antibodies}

Primary antibodies (Dilutions are given for immunoblotting unless mentioned)

\begin{tabular}{|c|c|c|c|c|}
\hline Target & Clone & $\begin{array}{l}\text { Source } \\
\text { organism }\end{array}$ & Dilution & Company \\
\hline ATR & $\mathrm{N}-19$ & goat & $1: 300$ & $\begin{array}{l}\text { Santa Cruz Biotechnology, Santa } \\
\text { Cruz, CA, United States }\end{array}$ \\
\hline ATRpT1989 & & rabbit & $1: 300$ & Kerafast \\
\hline BrdU/CldU & $\begin{array}{l}\text { BU1/75 } \\
(\text { ICR1) }\end{array}$ & rat & $\begin{array}{l}\text { 1:500 for } \\
\text { immunostaining }\end{array}$ & $\begin{array}{l}\text { AbDSerotec, MorphoSys, } \\
\text { Martinsried, Germany }\end{array}$ \\
\hline BrdU/IdU & B44 & mouse & $\begin{array}{l}\text { 1:500 for } \\
\text { immunostaining }\end{array}$ & Becton Dickinson \\
\hline Cdc7 & DCS341 & mouse & $1: 1,000$ & Abcam \\
\hline Cdc7 & SPM171 & mouse & $1: 1,000$ & $\begin{array}{l}\text { Santa Cruz Biotechnology, Santa } \\
\text { Cruz, CA, United States }\end{array}$ \\
\hline Chk1 & 2G1D5 & mouse & $1: 1,000$ & Cell Signaling Technology \\
\hline Chk1pS317 & & rabbit & $1: 1,000$ & Cell Signaling Technology \\
\hline Chk2pT68 & $\mathrm{C} 13 \mathrm{C} 1$ & rabbit & $1: 1,000$ & Cell Signaling Technology \\
\hline Claspin & & rabbit & $1: 1,000$ & Cell Signaling Technology \\
\hline H2AX pS319 & & rabbit & $1: 1,000$ & Cell Signaling Technology \\
\hline $\mathrm{Hsc} 70$ & B-6 & mouse & $1: 10,000$ & $\begin{array}{l}\text { Santa Cruz Biotechnology, Santa } \\
\text { Cruz, CA, United States }\end{array}$ \\
\hline Hsp27 pS82 & & rabbit & $1: 1000$ & Cell Signaling Technology \\
\hline $\lg G$ & & rabbit & $3 \mu \mathrm{G}$ for pulldown & Abcam \\
\hline JNK pT183/Y185 & & rabbit & $1: 1,000$ & Cell Signaling Technology \\
\hline Mcl-1 & & rabbit & $1: 1,000$ & Cell Signaling Technology \\
\hline MCM2pS53 & EP4120 & rabbit & $1: 10,000$ & Abcam \\
\hline MKP1 & & rabbit & $1: 1,000$ & Millipore \\
\hline Myc-tag & $4 \mathrm{~A} 6$ & mouse & $1: 1,000$ & Millipore \\
\hline р38pT180/Y182 & & rabbit & $1: 1,000$ & Cell Signaling Technology \\
\hline p53 & DO-1 & mouse & $1: 1,000$ & $\begin{array}{l}\text { Santa Cruz Biotechnology, Santa } \\
\text { Cruz, CA, United States }\end{array}$ \\
\hline$\beta$-Actin & AC-15 & mouse & $1: 20,000$ & Abcam \\
\hline$\beta$-Galactosidase & & mouse & $3 \mu \mathrm{G}$ for pulldown & Promega \\
\hline
\end{tabular}




\subsection{Secondary antibodies}

\begin{tabular}{|c|c|c|}
\hline Antibody & Cat. Number & Company \\
\hline Alexa-Fluor-488 goat anti mouse & A-11017 & Invitrogen, Life Technologies \\
\hline Alexa-Fluor-555 goat anti rat & A-21434 & Invitrogen, Life Technologies \\
\hline $\begin{array}{l}\text { HRP-coupled AffiniPure } F\left(a b^{\prime}\right) 2 \\
\text { fragment, anti mouse } \lg G(\mathrm{H}+\mathrm{L})\end{array}$ & $711-036-152$ & Jackson Immunoresearch \\
\hline $\begin{array}{l}\text { HRP-coupled AffiniPure } F\left(a b^{\prime}\right) 2 \\
\text { fragment, anti rabbit } \lg G(\mathrm{H}+\mathrm{L})\end{array}$ & $715-036-150$ & Jackson, Immunoresearch \\
\hline
\end{tabular}

\subsection{Human cell lines}

\begin{tabular}{ll} 
Cell line & Type \\
\hline Jurkats & Acute T-cell leukemia \\
MIA PaCa-2 & Pancreatic adenocarcinoma \\
U2OS & Osteosarcoma
\end{tabular}

\subsection{Media and reagents for eukaryotic cell culture}

Reagent

Ciprofloxacin

Dulbecco's Modified Eagle Medium (DMEM), powder

Fetal Calf Serum (FCS)

L-Glutamine

PBS (tablets)

Penicillin/Streptomycin

RPMI Medium

Tetracyclin

Trypsin/EDTA

\section{Company}

Bayer

Gibco, Life Technologies

Gibco, Life Technologies

Gibco, Life Technologies

Gibco, Life Technologies

Gibco, Life Technologies

Gibco, Life Technologies

Gibco, Life Technologies

Gibco, Life Technologies

\section{DMEM}

\begin{tabular}{lr}
\hline DMEM, powder & $10.0 \mathrm{~g}$ \\
$\mathrm{NaHCO}_{3}$ & $3.7 \mathrm{~g}$ \\
$\mathrm{HEPES}$ & $5.96 \mathrm{~g}$ \\
dissolved in $\mathrm{H}_{2} \mathrm{O}$ &
\end{tabular}




\subsection{Bacteria strain}

\begin{tabular}{lll} 
Strain & Description & Company \\
\hline $\mathrm{DH} 10 \mathrm{~B}$ & Chemically competent E.coli & Self made
\end{tabular}

\subsection{Bacteria culture media}

2YT medium

\begin{tabular}{ll}
\hline Tryptone & $1.6 \%$ \\
Yeast extract & $1.0 \%$ \\
$\mathrm{NaCl}$ & $0.5 \%$
\end{tabular}

2YT agar

\begin{tabular}{lr}
\hline YT agar & $15 \%$ \\
2YT medium & $100 \%$
\end{tabular}

\subsection{Software}

\begin{tabular}{ll} 
Name & Company \\
\hline CFX Manager Software for & Bio-Rad \\
qPCR cycler & \\
Excel & Microsoft, Redmond, WA, United States \\
Graph Pad Prism & GraphPad Software, Inc. \\
INTAS labID & Intas Science Imaging Instruments \\
Nanodrop Software & Peqlab \\
Fiji & General Public License \\
Microscoft picture manager & Microsoft
\end{tabular}




\section{Methods}

\subsection{Cell biology}

\subsubsection{Culturing of human cells}

All cell culture work was performed under laminar flow hoods and sterile conditions were maintained. Hoods were always cleaned with $70 \%$ ethanol prior to usage and only autoclaved pipettes and tips were used. The reagents used for cell culture were prepared and opened under the hood. Adherent U2OS cells were grown at $37{ }^{\circ} \mathrm{C}$ with $5 \% \mathrm{CO}_{2}$, in coated petri dishes using pre-warmed DMEM full medium (composition given in Table 4.1). For sub-culture, cells were washed once with 1X PBS and trypsinized for 5 to 7 min using $0.1 \%$ trypsin/EDTA. This led to the detachment of cells from the plate after which an equal volume of DMEM was added to inactivate trypsin. Afterwards, these floating cells were put in a falcon and centrifuged at 1000 rpm for 5 min, supernatant was discarded and cells were resuspended in full culture medium. Depending on the requirement, cells were reseeded at dilutions of $1: 5$ to $1: 10$, two to three times per week. For experiments, cells were counted using a Neubar chamber and re-seeded at an appropriate density in either 6-well plates (for all immunoblot and IP experiments) or $50 \mathrm{~mL}$ flasks (for all the DNA fiber assays).

Table 4.1 Culture Medium.

\begin{tabular}{|l|c|}
\hline Cell line & Medium composition \\
\hline U2OS & DMEM \\
\hline & $10 \% F C S$ \\
\hline & $50 \mathrm{U} / \mathrm{mL}$ penicillin \\
\hline & $50 \mathrm{U} / \mathrm{mL}$ streptomycin \\
\hline & $200 \mu \mathrm{M} \mathrm{L-glutamine}$ \\
\hline & $10 \mu \mathrm{g} / \mathrm{ml}$ ciprofloxacin \\
\hline
\end{tabular}

\subsubsection{Freezing of cells}

Cells were frozen and stored for later usage. For this purpose, low-passage (passage number 3 or 4 ) cells were grown in a $15 \mathrm{~cm}$ dish to full confluence and later trypsinized in the same manner as described above (4.1.1). All the cells were centrifuged at $1000 \mathrm{rpm}$ for 5 min, supernatant was removed and cells were re-suspended in pre-cooled freezing medium (FCS with 10\% DMSO). The cell suspension was transferred to cryo vials, which was immediately kept in a pre-cooled isopropanol box at $-80^{\circ} \mathrm{C}$ to allow slow cooling, and $24 \mathrm{~h}$ later shifted to liquid nitrogen tanks. 


\subsubsection{Thawing of frozen cells}

Cells frozen as described above were thawed when required. To this end, aliquots were held under lukewarm running water $\left(37^{\circ} \mathrm{C}\right)$ for around a minute, and rapidly transferred into a 50 $\mathrm{mL}$ flacon containing pre-warmed full medium. The cells were centrifuged for $5 \mathrm{~min}$ at 1000 rpm, re-suspended in fresh medium and seeded in a $5 \mathrm{~cm}$ petri dish.

\subsubsection{Inhibitor and chemotherapeutic treatment}

Chemotherapeutics and inhibitors were ordered in powder form and later dissolved (under the cell culture hood) in an appropriate solvent at the desired stock concentration (Table 4.2). For experiments, these drugs were used at the concentrations given in Table 4.2, by dissolving the required volumes from the stock in medium. An equivalent volume of the solvent was added in the control. In cases when volumes were lower than $0.4 \mu \mathrm{L}$, a master mix of the drug/solvent was prepared in medium and used.

Table 4.2 Concentrations of inhibitors and chemotherapeutics used in cell culture.

\begin{tabular}{|l|c|c|c|}
\hline Chemical & Solvent & Stock & $\begin{array}{c}\text { Final } \\
\text { concentration }\end{array}$ \\
\hline BCl (MKP1 Inh) & DMSO & $10 \mathrm{mM}$ & $1 / 3 / 5 \mu \mathrm{M}$ \\
\hline Gemcitabine & $\mathrm{H}_{2} \mathrm{O}$ & $64 \mathrm{mM}$ & $400 / 500 \mathrm{nM}$ \\
\hline KU55933 (ATM Inh) & DMSO & $10 \mathrm{mM}$ & $10 \mu \mathrm{M}$ \\
\hline MG132 (Proteasome Inh) & DMSO & $10 \mathrm{mM}$ & $10 / 20 \mu \mathrm{M}$ \\
\hline MK2III (MK2 Inh) & DMSO & $10 \mathrm{mM}$ & $10 \mu \mathrm{M}$ \\
\hline Z-VAD-FMK (Caspase inh) & DMSO & $10 \mathrm{mM}$ & $25 / 40 \mu \mathrm{M}$ \\
\hline
\end{tabular}

\subsubsection{Transfection of human cells}

\subsubsection{Transient transfection with siRNAs}

We employed RNA interference strategy to silence our gene of interest. This was achieved by transient reverse transfection of U2OS cells with small interfering RNA's (siRNA) using a lipid based transfection agent, lipofectamine 2000. For carrying out this procedure, lipofectamine and siRNA were dissolved separately in DMEM only medium (without FCS, Glu and antibiotics) as outlined below (Table 4.3), and incubated at room temperature (RT) for 5 min. 
Table 4.3 Concentration and volume of siRNA and lipofectamine used for transient transfections.

\begin{tabular}{|l|c|c|c|}
\hline MKP1 siRNA & DMEM only & Lipofectamine 2000 & DMEM only \\
\hline $0.4 \mu \mathrm{L}$ for $10 \mathrm{nM}$ siRNA & $200 \mu \mathrm{L}$ & $4 \mu \mathrm{L}$ & $200 \mu \mathrm{L}$ \\
\hline $0.6 \mu \mathrm{L}$ for $15 \mathrm{nM}$ siRNA & $200 \mu \mathrm{L}$ & $6 \mu \mathrm{L}$ & $200 \mu \mathrm{L}$ \\
\hline
\end{tabular}

Later, they were combined and incubated for another 20 to 40 min at RT. In the meantime, a 80\% confluent U2OS plate was trypsinized; cells were collected in a falcon and counted. In one well of a 6 -well plate, around 280,000 cells were seeded in $1.6 \mathrm{~mL}$ DMEM full medium (well-surface: $3.6 \mathrm{~cm}^{2}$ ); and $400 \mu \mathrm{L}$ of the prepared lipofectamine-siRNA mix was added drop-wise. Media was changed after $24 \mathrm{~h}$, and cells were grown in full medium without siRNA for an additional $24 \mathrm{~h}$. For the experiments which involved the use of certain inhibitors, details have been mentioned in their figure legends.

\subsubsection{Transient transfection with expression vectors}

To over-express a protein, we used expression vectors encoding the complementary DNA (CDNA) sequence of the desired gene and did forward transfection with lipofectamine 2000. For this, $-320,000$ U2OS cells were seeded per well in a 6-well plate and incubated overnight at $37{ }^{\circ} \mathrm{C}$ with $5 \% \mathrm{CO}_{2}$. Next day, the desired amount of plasmid DNA and lipofectamine 2000 were separately dissolved in DMEM only medium (Table 4.4) for 5 min, then mixed, incubated for 20 to $40 \mathrm{~min}$ at RT. The media from the cells was sucked off and the prepared $400 \mu \mathrm{L}$ DNA-lipofectamine mix was added to the cells drop-wise. Media was changed after $4 \mathrm{~h}$ and experiments were performed (Co-IP) $24 \mathrm{~h}$ after transfection. The details of Co-IP are explained in 4.2.6.

Table 4.4 Concentration and volume of siRNA and lipofectamine used for transient transfections.

\begin{tabular}{|l|c|c|c|}
\hline Plasmid & DMEM only & Lipofectamine 2000 & DMEM only \\
\hline 375 nG DNA & $200 \mu \mathrm{L}$ & $1 \mu \mathrm{L}$ & $200 \mu \mathrm{L}$ \\
\hline $0.8 \mu \mathrm{G}$ DNA & $200 \mu \mathrm{L}$ & $2 \mu \mathrm{L}$ & $200 \mu \mathrm{L}$ \\
\hline $1.6 \mu \mathrm{G}$ DNA & $200 \mu \mathrm{L}$ & $4 \mu \mathrm{L}$ & $200 \mu \mathrm{L}$ \\
\hline $2.4 \mu \mathrm{G}$ DNA & $200 \mu \mathrm{L}$ & $6 \mu \mathrm{L}$ & $200 \mu \mathrm{L}$ \\
\hline
\end{tabular}

In the experiments where siRNA and plasmid transfections were combined, cells were first reverse transfected with siRNA and $24 \mathrm{~h}$ later forward transfected with the plasmid DNA of interest. After $4 \mathrm{~h}$ of plasmid transfection, media was changed. Cells were grown in full medium for an additional $24 \mathrm{~h}$ and later harvested for SDS-PAGE analysis as described below. 


\subsection{Biochemistry techniques}

\subsubsection{Preparation of cell lysates for protein separation by SDS-PAGE}

To make whole cell lysates, cells from 6-well plates were scrapped off in medium by gentle strokes using a $25 \mathrm{~cm}$ rubber cell scrapper and transferred to $1.5 \mathrm{~mL}$ reaction tubes, on ice. This was followed by centrifugation at $4600 \mathrm{rpm}, 4{ }^{\circ} \mathrm{C}$ for $10 \mathrm{~min}$. The resulting supernatant was sucked off using a vacuum pump and pellet was washed once with PBS. This was subjected to a second round of centrifugation like before, and the resulting pellet was lysed using $100 \mu \mathrm{L}$ RIPA or Co-IP lysis buffer (with protease inhibitors) depending on the experiment. Further, lysates were shaken at $1000 \mathrm{rpm}$ for $15 \mathrm{~min}$ in the cold room to shear DNA; which was later spun down at $13000 \mathrm{rpm}, 4{ }^{\circ} \mathrm{C}$ for $10 \mathrm{~min}$. The supernatant containing all the proteins was utilized for protein estimation using the bicinchoninic acid (BCA) assay. For loading onto gels, appropriate volume of protein lysate was taken into a new reaction tube and a desired volume of $6 x$ laemmli buffer was added such that the final concentration of laemmli was $1 \mathrm{x}$. Lysates were shaken at $95{ }^{\circ} \mathrm{C}$ for $5 \mathrm{~min}$ to denature the proteins, and quickly spun down using a mini table top centrifuge.

\subsubsection{Bicinchoninic acid (BCA) assay for protein estimation}

Protein estimation was done using Pierce BCA Protein Assay Kit. This method takes advantage of a protein's ability to reduce copper from its $\mathrm{Cu}^{+2}$ to $\mathrm{Cu}^{+1}$ state in an alkaline environment. In the first step, peptides react with $\mathrm{Cu}^{+2}$ ions and reduce it to $\mathrm{Cu}^{+1}$ under the influence of an alkaline medium provided by sodium potassium tartrate. This yields a light blue color. To increase the sensitivity of this reaction for an easier detection, a second step converts the pale blue color to dark purple where two molecules bicinchoninic acid (BCA) react with one $\mathrm{Cu}^{+1}$ forming a water soluble $\mathrm{BCA}$-copper complex which absorbs light strongly at $562 \mathrm{~nm}$. To perform the BCA test, we followed the protocol as given in the user manual. For one reaction cocktail, reagent $A$ and $B$ were mixed in a ratio of $98: 2$ to which 5 $\mu \mathrm{L}$ protein lysate was added and incubated at $37^{\circ} \mathrm{C}$ for $30 \mathrm{~min} .2 \mu \mathrm{L}$ of the resultant dark purple complex was measured at $562 \mathrm{~nm}$ using a nanodrop spectrophotometer. As a control, $5 \mu \mathrm{L}$ lysis buffer was used instead of the protein. A BSA standard curve was also prepared in a similar way, and the unknown protein concentration was calculated using this reference.

\subsubsection{Separating proteins using SDS-PAGE}

Electrophoretic mobility of proteins can be exploited to separate them on a polyacrylamide gel according to their molecular weight. This is called PAGE (Polyacrylamide Gel Electrophoresis). However, in 1970, U. K. Laemmli refined this technique using denaturing

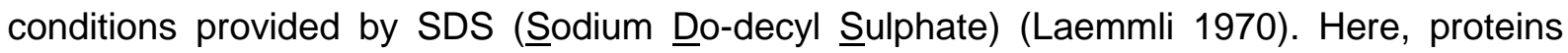
were boiled in laemelli buffer containing SDS and a reducing agent (DTT or $\beta$ - 
mercaptoethanol). While reducing agents were used to break the disulfide bonds; anionic SDS binds to proteins in amounts proportional to its molecular mass and imparts an overall negative charge. This denaturation ensured the separation of proteins according to their molecular mass. During electrophoresis, all proteins moved towards anode due to their negative charges but their migration rate was different depending on their mass. Heavier proteins traveled much slower as compared to the lighter proteins which moved faster through the pores of acrylamide. The discontinuous acrylamide gel was composed of two layers - an upper $5 \%$ stacking gel with a $\mathrm{pH}$ of 6.8 ; and a lower resolving gel of $\mathrm{pH} 8.8$ with a higher percentage of acrylamide (12\%). The lower percentage stacking gel having big pores, allowed all the proteins to travel at almost the same rate and 'stack' together as a single band before entering the lower gel, where the proteins separation took place. Table 4.5 summarizes the composition of the gels. $10 \mu \mathrm{G}$ of protein lysate was loaded on the gel after BCA estimation along with a pre-stained protein ladder to monitor protein separation and to estimate their size. The gels were run in SDS running buffer at a constant voltage of $100 \mathrm{~V}$ for $3 \mathrm{~h}$.

Table 4.5 Composition for one gel.

\begin{tabular}{|l|c|c|}
\hline Components & $\begin{array}{c}\text { Stacking gel 5\% } \\
(\mathbf{m L})\end{array}$ & $\begin{array}{c}\text { Resolving gel 12\% } \\
(\mathbf{m L})\end{array}$ \\
\hline $\mathrm{ddH}_{2} \mathrm{O}$ & 8.4 & 13.2 \\
\hline Acrylamide/Bisacrylamide & 2 & 16 \\
\hline Tris $\mathrm{pH} 6.8$ & 1.52 & - \\
\hline Tris $\mathrm{pH} 8.8$ & - & 10 \\
\hline $10 \% \mathrm{SDS}$ & 0.12 & 0.4 \\
\hline $10 \% \mathrm{APS}$ & 0.12 & 0.4 \\
\hline TEMED & 0.012 & 0.019 \\
\hline
\end{tabular}

\subsubsection{Immunoblotting}

Immunoblotting or Western blotting is an analytical technique developed in 1979 by Towbin and his co-workers for the detection of proteins (including post translational modifications and protein-protein interactions) after separating them on a SDS-PAGE (Towbin, Staehelin et al. 1979). By applying current, proteins are transferred from the gel to a nitrocellulose membrane. Once on the membrane, proteins are detected using a specific primary antibody raised against a particular epitope of the protein. Subsequently, a secondary antibody coupled to a horseradish peroxidase enzyme is added to the membrane which is capable of binding to the constant region of first antibody thus making it possible for a species-specific recognition. The enzyme oxidizes its substrate, luminol, in the presence of $\mathrm{H}_{2} \mathrm{O}_{2}$ to produce light which can be recorded by a camera. Since the light emitted is proportional to the amount of enzyme bound, a rough estimation of the amount of protein can be made. 
In our lab we used the method of wet western blotting to detect proteins. In this technique, a stack of whattmann filter paper, gel and nitrocellulose membrane was assembled. The gel was kept facing the anode side (plus end) to allow for transfer onto the membrane. This sandwich was kept in a running chamber with pre-cooled transfer buffer, and run at a constant voltage of $100 \mathrm{~V}$ for $2 \mathrm{~h}$ at $4^{\circ} \mathrm{C}$. Once transfer was done, membrane was stained with Ponceau-S and a picture was taken. This allowed us to cut the membrane into small pieces to stain for different proteins. Ponceau-S stain was washed off with transfer buffer. Later, membrane was blocked for $1 \mathrm{~h}$, at RT with 5\% milk prepared in TBS-T, washed twice with TBST and incubated overnight at $4^{\circ} \mathrm{C}$ with different primary antibodies prepared in $5 \%$ BSA (made in TBS-T). Next day, the membranes were washed three times with TBST and blocked with milk for 15 min to avoid unspecific binding by the secondary antibody. This was followed by the addition of secondary antibody for 1-2 h, at RT (donkey anti mouse or donkey anti rabbit; prepared in milk). Thereupon, membranes were blocked with milk for 15 min and washed thrice with TBST.

Detection was made possible by using the Immobilon Western Chemiluminescent HRP Substrate (Millipore) or SuperSignal West Femto Maximum Sensitivity Substrate (Thermo Scinetific). For every $4 \mathrm{~cm} * 5 \mathrm{~cm}$ membrane, $500 \mu \mathrm{L}$ of HRP substrate was mixed with 500 $\mu \mathrm{L}$ of peroxide solution, added to the membrane and incubated for $2 \mathrm{~min}$. Luminescence was detected on INTAS Image using a Chemocam HR 163200 imager.

\subsubsection{Quantitative Reverse Transcription- Polymerase Chain Reaction (qRT- PCR)}

To monitor gene expression, mRNA levels were quantified using quantitative Reverse Transcription - Polymerase Chain Reaction (qRT- PCR). This required the isolation of total RNA from the cells, followed by its conversion into complementary DNA (cDNA) with the enzyme Reverse Transcriptase, and finally amplification of the cDNA using PCR. These steps are described in detail below.

\subsubsection{Total RNA isolation}

Total RNA was extracted from human cells using the readily available guanidiumisothiocyanate-phenol solution, TRIzol, under the fume hood. For each well of a 6-well plate, cells were washed with $1 \mathrm{~mL}$ PBS and later lysed using $1 \mathrm{~mL}$ TRIzol for $5 \mathrm{~min}$ at RT. Cells were carefully re-suspended for homogenization and transferred to a $2 \mathrm{~mL}$ eppendorf tube. To isolate RNA from the proteins, $200 \mu \mathrm{L}$ of chloroform (per $\mathrm{mL}$ of TRIzol) was added and shaken vigorously for $15 \mathrm{sec}$. This was centrifuged at $13,200 \mathrm{rpm}, 4^{\circ} \mathrm{C}$ for 45 to $60 \mathrm{~min}$. The resulting upper aqueous phase containing RNA was carefully separated without touching the 
interphase, and transferred into a clean $1.5 \mathrm{~mL}$ tube. RNA was precipitated from this phase using $500 \mu \mathrm{L}$ isopropanol per $1 \mathrm{~mL}$ TRIzol. Samples were mixed thoroughly and incubated at RT for 10 min after which they were centrifuged at $13,200 \mathrm{rpm}, 4^{\circ} \mathrm{C}$ for $60 \mathrm{~min}$. Supernatant was discarded and RNA pellet was washed twice with $1 \mathrm{~mL} 70 \%$ Ethanol and spun down at $9000 \mathrm{rpm}, 4{ }^{\circ} \mathrm{C}$ for $20 \mathrm{~min}$. RNA was air dried for 15 min under fume and dissolved in $15 \mu \mathrm{L}$ nuclease free water. Following this, they were incubated at $55^{\circ} \mathrm{C}$ with mild vortexing. RNA concentration was measured using the nanodrop and stored at $-80{ }^{\circ} \mathrm{C}$.

\subsubsection{Synthesis of cDNA (RT)}

Using viral M-MuLV reverse transcriptase, mix of anchored oligo- $\mathrm{dT}_{23} \mathrm{VN}$ primers and random nonamers; the isolated RNA was converted to complementary DNA. The reaction cocktail was prepared in clean PCR tubes as follows: $1 \mu \mathrm{g}$ of RNA was combined with $2 \mu \mathrm{L}$ of mixed primer, $0.5 \mu \mathrm{L}$ dNTP mix and volume was made up to $16 \mu \mathrm{L}$ with autoclaved water. This was heated at $70{ }^{\circ} \mathrm{C}$ for 5 min to remove RNA secondary structures; and put on ice after a brief spin in a table top micro centrifuge. Here, each sample was prepared in a duplicate. In one set of RNA samples, RT master mix was added ( $4 \mu \mathrm{L}$ mix per sample), and in the other set, the samples were mixed with a master mix which was prepared without the RT enzyme $(4 \mu \mathrm{L}$ mix per sample). Composition of master mix is given below (Table 4.6):

Table 4.6 Components of RT reaction master mix.

\begin{tabular}{|l|c|c|}
\hline Components & Volume $(\boldsymbol{\mu L})$ & $\begin{array}{c}\text { Master mix } \\
\text { (e.g. 8 samples) }\end{array}$ \\
\hline RT 10X Reaction Buffer & 2 & 17 \\
\hline RNase inhibitor (10 U) & 0.25 & 2.125 \\
\hline $\begin{array}{l}\text { M-MuLV Reverse Transcriptase (25 U) } \\
\text { Or autoclaved water }\end{array}$ & 0.125 & 1.062 \\
\hline Autoclaved water & 1.625 & 13.812 \\
\hline
\end{tabular}

All this work was performed in RNase-free surrounding on ice. After the samples were prepared, they were incubated at $42^{\circ} \mathrm{C}$ for $1 \mathrm{~h}$ for the reverse transcription to take place. Subsequently the enzyme was inactivated by heating the samples to $95{ }^{\circ} \mathrm{C}$ for $5 \mathrm{~min}$. Each sample was then diluted by the addition of $30 \mu \mathrm{L}$ nuclease free water. DNA concentration was measured using the nanodrop and stored at $-20^{\circ} \mathrm{C}$.

\subsubsection{Amplification of cDNA using quantitative real time PCR}

cDNA generated in the previous step was amplified and detected in real time as the reaction progressed. This was achieved by the use of a fluorescent dye - SyBr Green in our experiments, which intercalated into the DNA and was used to measure the amount of DNA generated after every replication cycle. To calculate relative abundance of the gene of 
interest, a reference gene was chosen whose expression levels remained constant under various conditions (36B4 in our experiment). Later, all the values obtained from the gene of interest were normalized to this reference gene. To specifically amplify cDNA arising from the mRNA of our interest, sequence-specific primers were designed. Since the sensitivity of PCR is best when the product size is between $150-300 \mathrm{bp}$, forward and reverse primers were generated spanning the exon-junctions in such a way that only a small fragment of the cDNA was amplified. For one reaction mix, the components were mixed as given in the table below. To keep things simple, a master mix of all the components (without the cDNA) was prepared and $24 \mu \mathrm{L}$ of this master mix was added to $1 \mu \mathrm{L}$ of cDNA. cDNA resulting from RT reactions without reverse transcriptase and QPCR samples without CDNA template served as controls. All samples were analyzed in triplicates. The qPCR reaction mix and the qPCR cycler program are detailed below in Table 4.7 and 4.8 .

Table 4.7 Components of qPCR mix for one sample.

\begin{tabular}{|l|c|}
\hline Components & Volume $(\mu \mathrm{L})$ \\
\hline 25X qPCR reaction mix & 14 \\
\hline Forward primer $(10 \mathrm{pmol} / \mu \mathrm{L})$ & 0.75 \\
\hline Reverse primer $(10 \mathrm{pmol} / \mu \mathrm{L})$ & 0.75 \\
\hline cDNA & 1 \\
\hline Nuclease free water & 8.5 \\
\hline
\end{tabular}

Table 4.8 Cycler program for qPCR.

\begin{tabular}{|l|c|c|}
\hline Temperature & Time & \\
\hline $95^{\circ} \mathrm{C}$ & $2 \mathrm{~min}$ & \\
\hline $95^{\circ} \mathrm{C}$ & $15 \mathrm{sec}$ & \multirow{2}{*}{$40 \mathrm{X}$} \\
\hline $60^{\circ} \mathrm{C}$ & $1 \mathrm{~min}-\mathrm{read}$ & 40 \\
\hline
\end{tabular}

The fluorescence of each sample was measured after every cycle at the end of elongation ("read"). Purity of the qPCR product was controlled with a melting curve that should yield a single melting point for a specific product. The resulting $C_{t}$ values (amplification cycle at which the fluorescence reaches the determined threshold) were used for the calculation of the relative amount of template using the $\Delta \Delta \mathrm{C}_{\mathrm{t}}$ method, assuming $100 \%$ amplification efficiency (i.e. a product doubling with each cycle): relative mRNA expression $=2(\Delta \mathrm{Ct}$ ref. gene treated/target gene treated) - ( $\Delta \mathrm{Ct}$ ref. gene untreated/target gene untreated) 


\subsubsection{Co-immunoprecipitation}

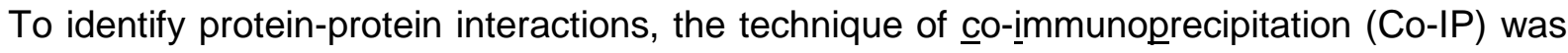
used. In this method, target proteins are first captured using specific antibodies, which is later analyzed for the interacting partner. Here, cell lysates are first incubated with the antibody against the protein of interest, which helps to precipitate it. In this manner, the proteins bound to the target proteins are also precipitated, in a complex. After this step, the complex is made to bind with Protein A or Protein G coupled sepharose beads. Protein A and Protein $G$ are bacterial proteins which can bind to the constant region of antibodies, thus facilitating the pull down of the antibody-target protein complex. The proteins which do not bind to the beads are washed away after multiple steps of washing, leaving a purified protein complex. These precipitated proteins are later identified with a SDS-PAGE followed by an immunoblot (see 4.2.3 and 4.2.4). Co-IP can be done in a native system or in an over-expression system using plasmids to over-express the proteins whose interaction needs to be monitored. To look for a possible interaction between MKP1 and Mcl-1, over-expressing plasmids of both proteins were used and a Co-IP was performed. On the day before the experiment, U2OS cells were seeded at a density of 280,000 cells per well of a 6 -well plate. Next day, cells were transiently transfected for $24 \mathrm{~h}$ with pcDNA3; Flag tagged hMcl-1 (Morel, Carlson et al. 2009) and Myc-DDK tagged-hDUSP1 using lipofectamine, in the manner as described in Table 4.9. The hMcl-1 and hDUSP1 plasmids were on a CMV backbone.

Table 4.9 Amount of plasmids used for transient transfections in Co-IP.

\begin{tabular}{|l|c|}
\hline Plasmid & $\boldsymbol{\mu G}$ \\
\hline pcDNA3 & 2.4 \\
\hline hMcl-1 + pcDNA3 & $0.8+1.2$ \\
\hline hDUSP1 + pcDNA3 & $1.6+0.8$ \\
\hline hMcl-1 + hDUSP1 & $0.8+1.6$ \\
\hline
\end{tabular}

After transfection, cells were washed once with $5 \mathrm{~mL}$ ice cold PBS and harvested using 500 $\mu \mathrm{L}$ Co-IP buffer on ice. Cell lysates from three wells of the same condition was pooled together, and transferred to a $2 \mathrm{~mL}$ reaction tube. The cell lysate was homogenized by pushing it through a $26 \mathrm{G}$ insulin syringe for 5 times. This was followed by sonification of the cell lysate to destroy the DNA. Later, samples were centrifuged at $13,000 \mathrm{rpm}, 4^{\circ} \mathrm{C}$ for 15 min, to get rid of the cell debris. The resulting supernatant was transferred to a new reaction tube and incubated with $75 \mu \mathrm{L}$ of pre-washed protein G sepharose (PGS) for $1 \mathrm{~h}$ on a rotator at $4{ }^{\circ} \mathrm{C}$. This is called the pre-clearing step, which is essential to remove proteins that bind unspecifically to the beads. Pre-washing of PGS is done before by suspending the beads three times in Co-IP buffer with short spins in between for $2 \mathrm{~min}$ at $3000 \mathrm{rpm}, 4^{\circ} \mathrm{C}$. After incubating the lysate with beads, samples were centrifuged for 4 min at $3000 \mathrm{rpm}, 4{ }^{\circ} \mathrm{C}$, and 
supernatant was transferred into a new tube. Around 20-50 $\mu \mathrm{L}$ of lysate from each sample was taken out and mixed with $15 \mu \mathrm{L}$ of $6 \mathrm{x}$ laemelli, for the input control. Now, each of these samples was divided into two parts. For one of the experiments where MKP1 was pulled down, one part of each sample was incubated with mouse anti $\beta$-galactosidase and the other part was incubated with mouse anti Myc-tag. For the second experiment where Mcl-1 was pulled down, one part of each sample was incubated with rabbit anti IgG and to the other part rabbit anti Mcl-1 was added. The mouse anti $\beta$-galactosidase and rabbit anti IgG worked as negative controls for the pull down. Antibodies were used at a concentration of $3 \mu \mathrm{G}$. Then, $20 \mu \mathrm{L} 50 \%$ PGS was added to each sample and incubated at $4{ }^{\circ} \mathrm{C}$ for $1 \mathrm{~h}$ on a rotator. This step assisted the coupling of the beads to the antibody-protein complex. This complex was centrifuged for $2 \mathrm{~min}$ at $3,000 \mathrm{rpm}, 4{ }^{\circ} \mathrm{C}$, and supernatant was discarded. The beads were then washed with $800 \mu \mathrm{L}$ Co-IP lysis buffer for five times, with spins in between for 2 min at $3000 \mathrm{rpm}$. At the last washing step, the supernatant was carefully removed using a syringe. The pellet was re-suspended in $25 \mu \mathrm{L} 3 \mathrm{x}$ laemmli buffer and boiled for $5 \mathrm{~min}$ at $95{ }^{\circ} \mathrm{C}$, together with input controls. Samples were centrifuged to spin down the beads, and supernatant was used for loading on SDS PAGE Samples were analyzed by immunoblotting followed by chemiluminiscence detection. For the experiment where MKP1 was pulled down, rabbit anti Mcl-1 antibody was used for detection after blotting and in the experiment where Mcl-1 was pulled down, mouse-anti Myc antibody was used.

\subsection{Molecular biology}

\subsubsection{Heat-shock transformation of chemical competent bacteria}

Transformation is the process where chemically competent bacterial cells take up any exogenous material, like DNA. This method is used for rapid amplification of plasmid DNA. Chemical competent E.coli cells, DH10B were used for this purpose. In this procedure, $1 \mu \mathrm{L}$ of plasmid DNA was incubated with $50 \mu \mathrm{L}$ of these competent cells. This was incubated for $30 \mathrm{~min}$ on ice and later for $10 \mathrm{~min}$ at $37^{\circ} \mathrm{C}$. This heat-shock allows the uptake of DNA. After this, cells are again kept on ice for an additional $10 \mathrm{~min}$ and then $200 \mu \mathrm{L} 2 \mathrm{YT}$ media was added. These bacteria were incubated for $30-60 \mathrm{~min}$ at $37^{\circ} \mathrm{C}$ with shaking at $300 \mathrm{rpm}$. This allowed the bacteria to grow, and this culture was then used for plating on agar plates with appropriate antibiotics (Kanamycin $25 \mu \mathrm{G} / \mathrm{mL}$, Ampicillin $100 \mu \mathrm{G} / \mathrm{mL}$ ). pCMV-Flag-hMcl-1 was a gift from Roger Davis (Addgene plasmid \# 25392), pBabe-Flag hMcl-1 was a gift from Roger Davis (Addgene plasmid \# 25371), DUSP1 (Myc-DDK-tagged)-Human dual specificity phosphatase 1 (DUSP1) was ordered from Origene (plasmid RC205220, NM_004417). 


\subsubsection{Plasmid DNA isolation}

Plasmid DNA was isolated from a transformed bacterial culture which was grown the previous night in 2YT medium with appropriate antibiotics. To isolate and purify the plasmid DNA in large volumes, the PureYield ${ }^{\mathrm{TM}}$ Plasmid Midiprep System (Promega) was used according to the manufacturer's instructions. For a smaller volume the Invisorb Spin Plasmid Mini Kit Two (Invitec) was used according to the manual.

\subsubsection{Measurement of nucleic acid concentration}

DNA concentration was measured using a spectrophotometer, NanoDrop Spectrophotometer, ND-1000 (PeqLab). $2 \mu \mathrm{L}$ of sample was used to measure the absorption co-efficient at $260 \mathrm{nM}$. This was later used to calculate the corresponding DNA in the sample. Water was used as a blank. For measuring RNA, same procedure was followed.

\subsubsection{Sequencing of DNA}

The plasmid DNA obtained from midi prep, or from outside has to be sequenced before proceeding with the experiment. This was done using the BigDye ${ }^{\circledR}$ Terminator v3.1 Cycle Sequencing Kit. This is based on the method that was originally developed by Sanger and colleagues (Sanger, Nicklen et al. 1977). This method utilizes fluorescent dideoxynucleotides (ddNTPs) which lack the 3'-OH group. Thus, when such a ddNTP is incorporated, then the elongating chain terminates. This chain termination leads to the formation of PCR products of varied lengths, which are later separated in a gel matrix. The fluorescence from the ddNTPs of each fragment size is then used in sequence analysis. For the sequencing PCR, $100 \mathrm{ng}$ of plasmid DNA was mixed with $30 \mathrm{pmol}$ of the appropriate primers and $2 x$ sequencing mix. The sequencing mix was composed of polymerase, dNTPs and fluorescently-labeled ddNTPs. The program used for sequencing PCR is given in Table 4.10

Table 4.10 Cycler program for sequencing PCR.

\begin{tabular}{|c|c|c|}
\hline Temperature & Time & \\
\hline $96^{\circ} \mathrm{C}$ & $2 \min$ & \\
\hline $96^{\circ} \mathrm{C}$ & $10 \mathrm{sec}$ & \\
\hline $55^{\circ} \mathrm{C}$ & $15 \mathrm{sec}$ & $20 x$ \\
\hline $60^{\circ} \mathrm{C}$ & $4 \min$ & \\
\hline $12^{\circ} \mathrm{C}$ & pause & \\
\hline
\end{tabular}

The PCR product was purified by ethanol precipitation and resuspended in Hi-Di Formamide for sequencing using an ABI 3100 Automated Capillary Sequencer. 


\subsection{DNA fiber assay}

Replication dynamics was monitored using the DNA fiber assay, based on the protocol published by Jackson and Pombo (Jackson and Pombo 1998). In this assay, cells are first pulse-labeled with 5-Chloro-2'-deoxyuridine (CldU) and later with 5-lodo-2'-deoxyuridine (IdU). Since CldU and IdU are nucleoside analogs, they get incorporated into the replicating DNA strand and mark progressing replication forks. This is followed by cell harvest and lysis. DNA is spread on a glass slide and fixed with methanol/acetic acid. Subsequently, DNA is denatured using hydrochloric acid treatment to expose the single strands; blocked and stained with specific primary and fluorescent-dye-coupled secondary antibodies. Such an immunofluorescence based method allows the detection of labeled DNA tracks using a fluorescence microscope. The resulting fork structures are as shown in Fig. 4.1. Using this technique replication fork speed, frequency of origin firing and replication termination can be calculated. Replication fork speed is calculated using the uni-directional structures. If a treatment is applied before the first pulse label, the total track length of CldU and IdU is taken for subsequent calculations. When cells are pretreated with the appropriate drugs, it is also possible to analyze how origin firing is affected. To assess origin firing, the ongoing, bidirectional forks ( $1^{\text {st }}$ label origins) are counted, and later presented as a percentage of all red label structures. Since these are the origins which fired when the first label was applied, if the drug treatment had changed the rate of origin firing, it should be easily detectable by such quantification.

For performing the DNA fiber assay, a day before the experiment, $5 \times 10^{5}$ U2OS cells were grown exponentially in $25 \mathrm{~mL}$ flasks with $5 \mathrm{~mL}$ medium for 16-20 h. In addition, $25 \mu \mathrm{M}$ of CldU and $250 \mu \mathrm{M}$ IdU was prepared in medium by appropriate dilutions from the stock solutions. The diluted labels and a flask of fresh medium were left to equilibrate overnight at $37^{\circ} \mathrm{C}$ in the incubator. On the day of experiment, inhibitors and chemicals were diluted in the different media (CldU medium, IdU medium or fresh medium alone), depending on the experiment. For pre-treating cells, inhibitors were first diluted in the fresh medium prepared the previous night and left to incubate for approximately $15 \mathrm{~min}$ in the incubator. Subsequently, cells were taken out of the incubator, and its medium was aspirated. This was followed by the addition of the inhibitor containing medium, and cells were left to incubate for $1 \mathrm{~h}$ at $37^{\circ} \mathrm{C}$ in the incubator. During this time, inhibitors were added to the CldU and IdU containing media, and left to equilibrate at $37^{\circ} \mathrm{C}$. After $1 \mathrm{~h}$, the medium was aspirated and the CldU-inhibitor containing medium was added. After $30 \mathrm{~min}$ of CldU label, this medium was replaced with the IdU-inhibitor medium for an additional $30 \mathrm{~min}$. The IdU concentration used was ten times higher than CldU to ensure that CldU is not incorporated any more during the second label. When the experiment involved the use of gemcitabine, it was added 
only to the second label. After IdU treatment, cells were kept immediately on ice to stop further replication. Medium was aspirated, and cells were washed twice with ice cold PBS. Subsequently, cells were harvested in $2 \mathrm{~mL}$ PBS with gentle strokes using a $16 \mathrm{~mL}$ scrapper. Cells were collected in a $2 \mathrm{~mL}$ tube, and centrifuged at $4,400 \mathrm{rpm}$ at $4{ }^{\circ} \mathrm{C}$ for $7 \mathrm{~min}$. The resulting supernatant was removed using a needle and pellet was re-suspended in $100 \mu \mathrm{L}$ of PBS. Cells were counted and samples were diluted to obtain a final density of $5 \times 10^{5}$ cells per $\mathrm{mL} .2 \mu \mathrm{L}$ drop of each sample was placed at the top end of superfrost, glass slide. This was allowed to stand at RT for approximately 3-4 min, after which $7 \mu \mathrm{L}$ of spreading buffer was added and incubated for another $2 \mathrm{~min}$. After this the slide was tilted to form a nose, and the drop was made to run down the slide slowly at a speed of around $3 \mathrm{~cm} / \mathrm{min}$. In this way, the DNA is spread on the slide, which is left to air-dry for 30 min and later fixed in the fiber assay fixative for $10 \mathrm{~min}$. These slides can be stored up till one month at $4{ }^{\circ} \mathrm{C}$.

1

2

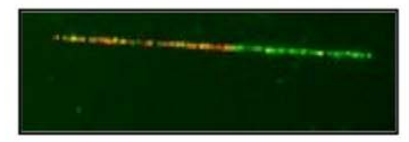

3

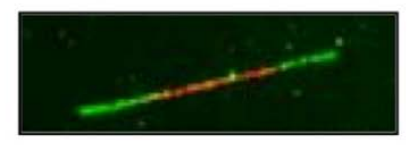

4

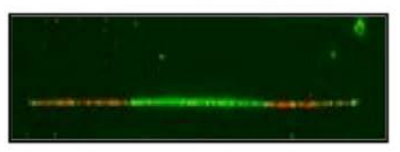

5

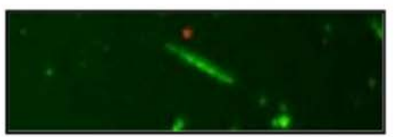

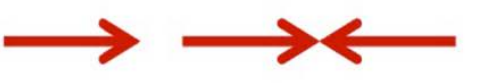
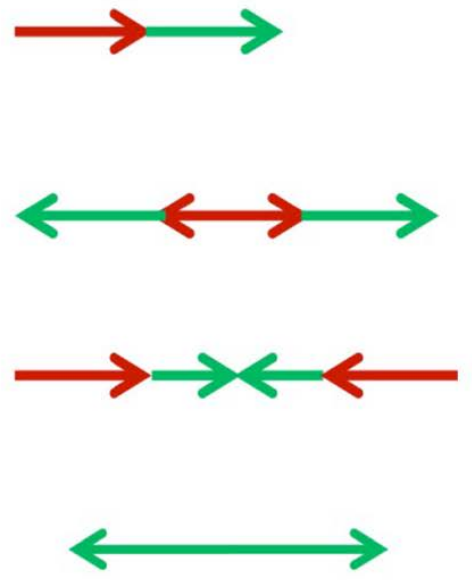

$1^{\text {st }}$ label origin

Fused forks/

$2^{\text {nd }}$ label termination

stalled fork/
1st label termination

ongoing fork

unidirectional

$2^{\text {nd }}$ label origin

\section{Figure 4.1 Representative images of the various structures analyzed in DNA fiber assay}

CldU was the first pulse label given to the cells, which was detected by a specific rat anti BrdU primary antibody and red fluorescent secondary antibody. Similarly, IdU was the second pulse label given, which was detected by mouse anti BrdU primary antibody and subsequently by a green fluorescent secondary antibody. Analysis was done using Fiji. Ongoing, uni-directional forks were measured for their lengths in pixels which was later used for calculating the rate by conversion to $\mu \mathrm{m}$ and kilo bases $(1 \mu \mathrm{m}=2.59 \mathrm{~kb})$. Origin firing was quantified by counting the $1^{\text {st }}$ label origins and representing it as a percentage of all red-labeled fibers (1-4). (Based on Köpper et al., PNAS 2013 and Petermann et al., PNAS 2010).

For immunostaining, wet chamber was used to avoid the drying of slides during the procedure. The fixed slides were first rehydrated in $\mathrm{H}_{2} \mathrm{O}$, twice for 5 min and then equilibrated with $2.5 \mathrm{M} \mathrm{HCl}$ for $5 \mathrm{~min}$. DNA was denatured to yield single strands by the incubation of 
these slides with $\mathrm{HCl}$ for an additional $80 \mathrm{~min}$ at $\mathrm{RT}$. After $\mathrm{HCl}$ treatment, the water in chamber was changed, and the slides were rinsed twice with PBS. This was followed by two short 5 min rinses with blocking solution. Then, slides were blocked for an additional hour at RT. Thereafter, slides were dried thoroughly to get rid of the blocking solution, and incubated with $150 \mu \mathrm{L}$ of the primary antibodies for $1 \mathrm{~h}$, at $37^{\circ} \mathrm{C}$. While rat anti BrdU recognized CldU, mouse anti IdU was directed against IdU. Both the antibodies were prepared at a dilution of 1:500 in blocking solution. After this, slides were rinsed three times with PBS and fixed in 500 $\mu \mathrm{L} 4 \%$ formaldehyde (prepared in PBS) at RT, for $10 \mathrm{~min}$, and then rinsed once again with PBS. Then, blocking solution was added three times for 5 min each to prevent the unspecific binding by the secondary antibody. Slides were dried again, and $150 \mu \mathrm{L}$ of fluorophorecoupled secondary antibodies was added to the slides. Alexa-Fluor-555 goat anti rat and Alexa-Fluor-488 goat anti mouse were made at 1:250 dilution in blocking solution. Slides were left to incubate with secondary antibodies at RT for $2 \mathrm{~h}$. Later, slides were rinsed twice with PBS, washed three times with blocking solution for 5 min each, and rinsed once again with PBS and $\mathrm{dd}_{2} \mathrm{O}$. Slides were mounted using one drop of $\mathrm{H}-1400$ vectashield, and sealed from the sides with nail polish. Fluorescence microscopy was performed using a $63 x$ objective in one experiment (Result 5.3 was obtained with a $63 x$ objective), and a $40 \mathrm{X}$ objective was used for all other experiments.

For each sample, at least twenty microscopic images were taken and nearly 500 structures were counted and analyzed from each experiment. Ongoing forks were measured for their lengths using Fiji, and the Cell Counter Plug-in from Fiji was utilized for counting the origin firing structures (Kurt De Vos, University of Sheffield, UK). The resulting data was further analyzed with Microsoft Excel and Graph Pad Prism.

\subsection{Statistical analysis}

Statistical analysis was carried out with Graph Pad Prism. Statistical significance values were determined using the unpaired, two-tailed student's t-test. Significance was assumed for $p$ values below 0.05. n.s. = not significant. $n$ in figure legends indicates the number of independent experiments. Additionally, this was cross-checked by Mann-Whitney test. When measuring the lengths of DNA fibers using Image $\mathrm{J}$, conversion factors used were: with a $63 \mathrm{X}$ objective, the conversion factor used was $10 \mu \mathrm{m}=9.8$ and with a $40 \mathrm{X}$ objective, the conversion factor used was $10 \mu \mathrm{m}=3.1$. 


\section{Results}

\subsection{Inhibition of MKP1, using $\mathrm{BCl}$, accumulates phospho-H2AX in various cell lines}

In recent years, MKP1 has been established as a potential candidate responsible for chemoresistance in pancreatic cancer, ovarian cancer and lung cancer; owing to its high expression levels (see 2.8). Since many of the commonly used chemotherapeutic agents execute their actions by causing DNA damage, they also instigate the DNA damage response (DDR), orchestrated by various kinases and phosphatases. The present study aims to investigate the role of MKP1 in such a scenario via the usage of a commercially available small molecule inhibitor, BCl.HCL (2-benzylidene-3-(cyclohexylamino)-1-Indanone hydrochloride) (Molina, Vogt et al. 2009). BCl was able to arrest MKP1 in its least-active conformation; thereby blocking the 'substrate-binding induced' phosphatase activity. A wide variety of cancer cell lines were chosen for this purpose, namely U2OS (osteosarcoma), Jurkats (T-cell leukemia) and MiaPaCa-2 (pancreatic carcinoma). While U2OS and MiaPaCa-2 were treated with DMSO or $5 \mu \mathrm{M} \mathrm{BCl}$ for $4 \mathrm{~h}$, Jurkats were treated with $1 \mu \mathrm{M} \mathrm{BCl}$ for $3 \mathrm{~h}$ and subsequently harvested. The $3 \mathrm{~h}$ time point was chosen for Jurkats as they started undergoing cell death after $4 \mathrm{~h}$ of $\mathrm{BCl}$ treatment. Whole cell lysates were analyzed by immunoblotting for phosphorylated p38MAPK and DNA damage marker - phospho-histone 2AX (Ser 139) (or $\mathrm{YH} 2 \mathrm{AX}$ ). As expected, the levels of dually phosphorylated p38MAPK (designated as pP38MAPK Thr 180/Tyr 182) were increased, confirming the efficiency of $\mathrm{BCl}$. Concomitantly, an accumulation of $\mathrm{yH} 2 \mathrm{AX}$ was also seen (Fig. 5.1). Taken together, the above results suggest a close involvement of MKP1 in the DDR network.

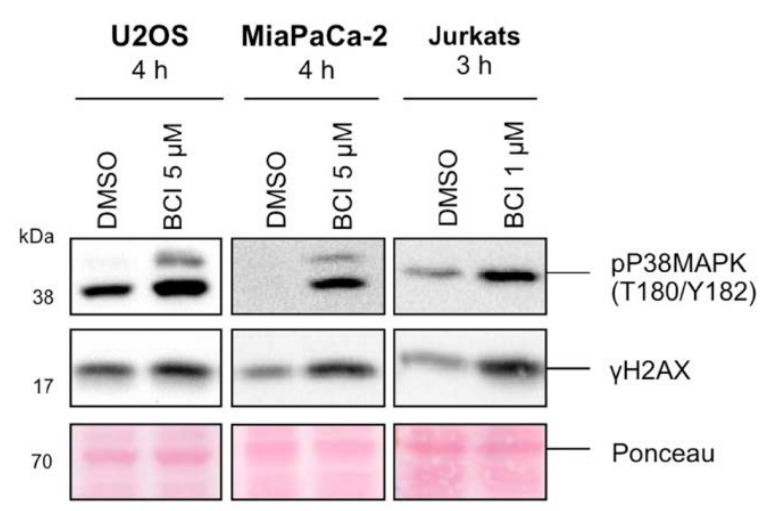

Figure 5.1 Accumulation of phospho-H2AX (S139) upon MKP1 inhibition using BCl.

MKP1 activity was blocked in U2OS, MiaPaCa-2 and Jurkats using the pharmacological allosteric inhibitor, $\mathrm{BCl}$. After treatment with the given concentrations of $\mathrm{BCl}$ or equal volumes of DMSO as a control for the indicated time points, cells were harvested; protein extracts were prepared and analyzed by immunoblotting using the indicated antibodies. Ponceau-S stain was used to check equal loading. 


\subsection{MKP1 inhibition impairs replication fork progression}

As the inhibition of MKP1 led to an accumulation of DNA damage even in the absence of any exogenous damaging agent (see 5.1), we were interested in elucidating the mechanistic details underlying this observation. One of the many reasons for such a response could be the existence of faulty replication, giving rise to spontaneous damage (Mazouzi, Velimezi et al. 2014). Hence, to evaluate replication kinetics, we used the DNA fiber assay as described in section 4.4. U2OS cells were selected as a model system for all the experiments due to their low levels of intrinsic DNA damage and convenience in handling.

For the assay, cells were incubated with MKP1 inhibitor, $\mathrm{BCl}$ or DMSO for $1 \mathrm{~h}$ and later pulse labeled with thymidine analogs, 5-Chloro-2'-deoxyuridine (CldU, first label) and 5-lodo-2'deoxyuridine (IdU, second label) for 30 min each, in the presence of DMSO or $\mathrm{BCl}$ (Fig. 5.2A). Following this, cells were lysed; DNA was spread on glass slides, denatured and immunostained with antibodies against CldU (red) and IdU (green), which was then, visualized using fluorescence microscopy. This technique allowed us to calculate the rate of replication by measuring the uni-directional, ongoing replication structures (red-green) for their lengths using (Fig. 5.2D; see 4.4). The analysis exhibited a significant reduction of fork speed with $\mathrm{BCl}$ treatment when compared to the DMSO control (Fig. 5.2B). This effect was also clearly evident in the frequency distribution of CldU and IdU fork speeds, where the $\mathrm{BCI}$ exposed population had a higher percentage of forks with lower fork rates (Fig. 5.2C), shifting the histogram to the left with reference to DMSO. Furthermore, given that MKP1 is nuclear; such an effect at DNA replication seems highly plausible.

DNA fiber assays can be conducted with siRNA mediated knockdowns of the protein, but have been shown to result in a steady state change of replication events as reported previously by Petermann and co-workers (Petermann, Woodcock et al 2010). Thus, we conducted experiments only using inhibitors for short periods of time that was able to induce profound changes, which could later be easily monitored.

In conclusion, the above results strongly indicate a role of MKP1 in supporting replication fork progression even in the absence of any exogenous DNA insults. 
A

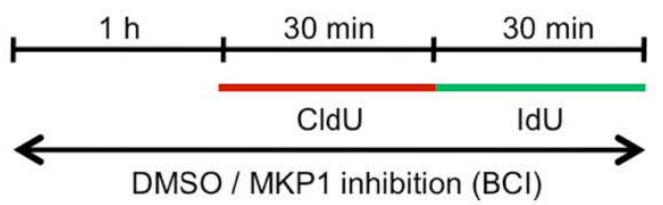

B

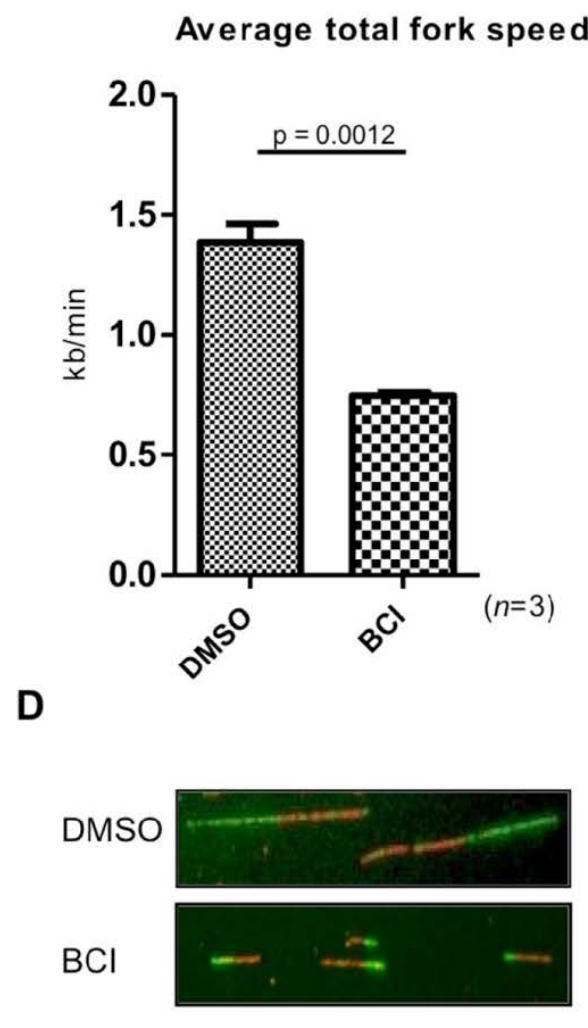

C

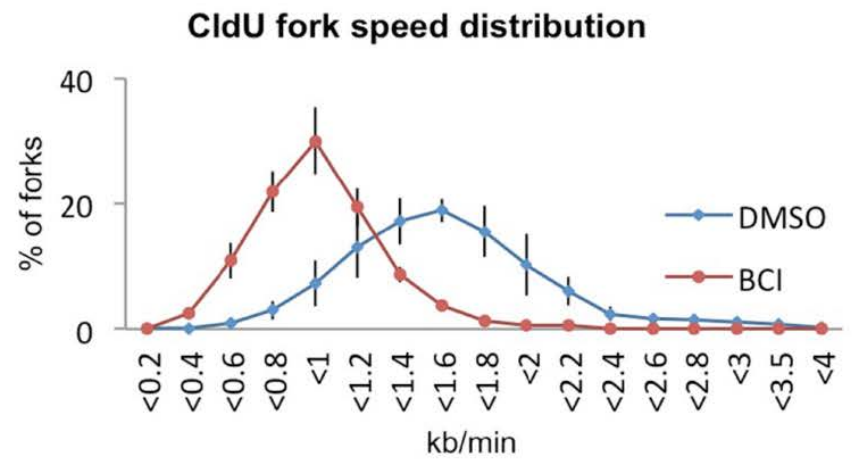

IdU fork speed distribution

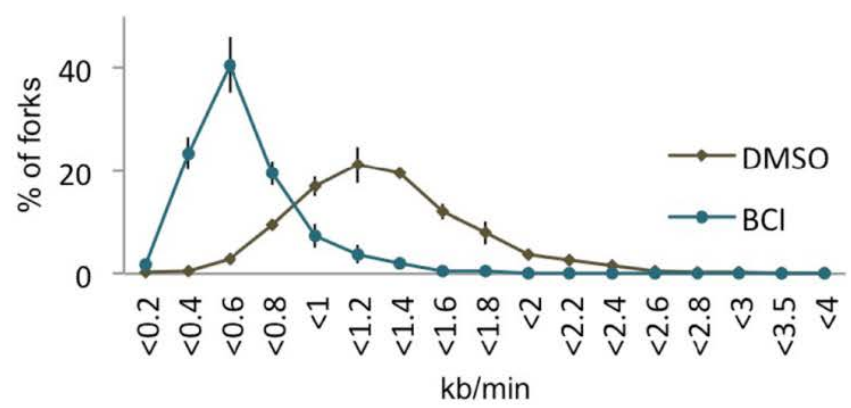

Figure 5.2 MKP1 inhibition reduces replication fork speed.

(A) Labeling protocol for DNA fiber analysis. U2OS cells were pre-treated with $3 \mu \mathrm{M} \mathrm{BCl}$ or an equal volume of DMSO for $1 \mathrm{~h}$. This was followed by pulse labeling with CldU or IdU for 30 min each, in the continuous presence of inhibitor. Both CldU and IdU were detected using specific primary antibodies. CldU was detected using red and IdU was detected using green secondary antibody.

(B) Average of total replication fork speed (sum of CldU and IdU labeled track lengths) in cells treated with DMSO and $\mathrm{BCl}$ as described in (A) (n=3). Statistical analysis was done using students t-test, $p$ values are indicated in the figures.

(C) Histogram depicting the distribution of CldU and IdU replication fork speeds in DMSO and $\mathrm{BCI}$ treated cells $(n=3)$.

(D) Representative images of replication tracks after labeling according to A. 


\subsection{MKP1 inhibitor acts together with Gemcitabine to further reduce replication fork speed}

After observing that MKP1 inhibition interferes with replication, (see 5.2), we wanted to explore the function of this phosphatase under conditions of replicative stress induced by exogenous sources.

In this view, we used the S-phase specific nucleoside analog, gemcitabine (2'-deoxy-2',2'difluorocytidine), which incorporates itself instead of cytidine into the replicating DNA, and thence leads to chain termination and fork stalling. Since both $\mathrm{BCl}$ and gemcitabine act by perturbing replication, we conducted DNA fiber experiments in their presence to examine these changes (experimental work done in collaboration with Dr. Ann Christin Parplys, Hamburg). Cells were treated as depicted in Fig. 5.3A and DNA fiber spreads were analyzed. As expected, in the single treatments of $\mathrm{BCl}$ and gemcitabine, there was a marked reduction in the replication fork speed. This was further reduced when the drugs were given in a combination treatment (Fig. 5.3B and D). Such a massive slow-down of replication raises the possibility of cells going into a prolonged S-phase arrest or pre-mature mitosis, either or both of which would ultimately result in cell death.

In this experiment, since gemcitabine was added at the second label, for comparison between samples, ideally, only IdU track lengths should have been taken. However, in our experiments, we saw that the addition of gemcitabine also affects the CldU label, where they become shorter when compared to the control. Thus, we chose to calculate the total fork speed using CldU and IdU track lengths. This phenomenon is likely due to the instability of forks upon the addition of gemcitabine (Schlacher, Christ et al. 2011, Schlacher, Wu et al. 2012). 
A

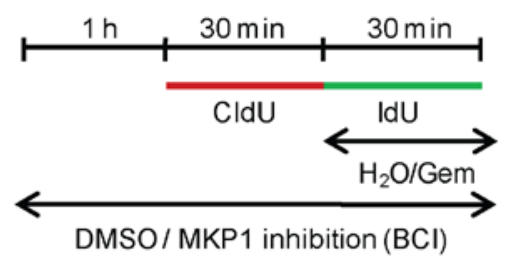

B

Average total fork speed

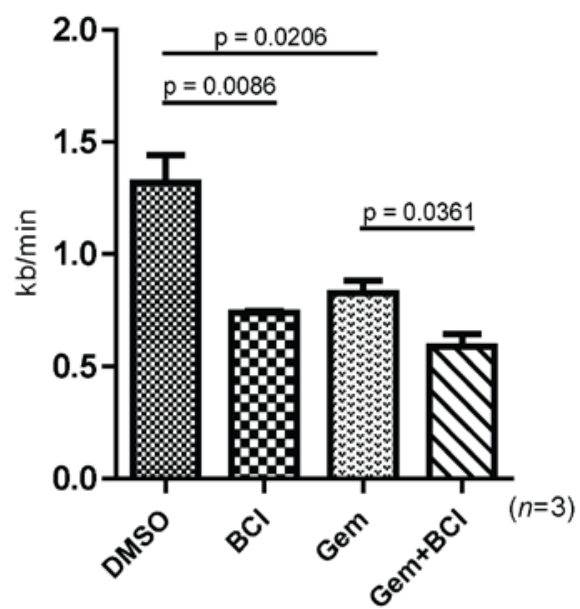

C

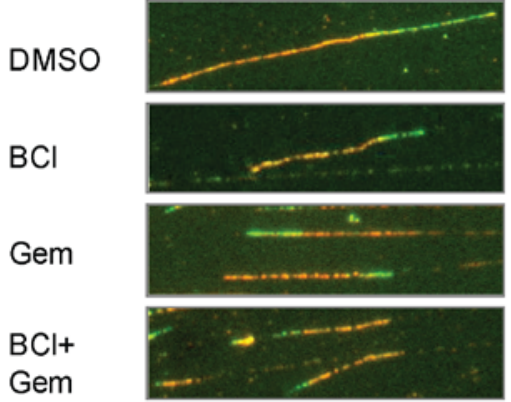

D

Distribution of total fork speed

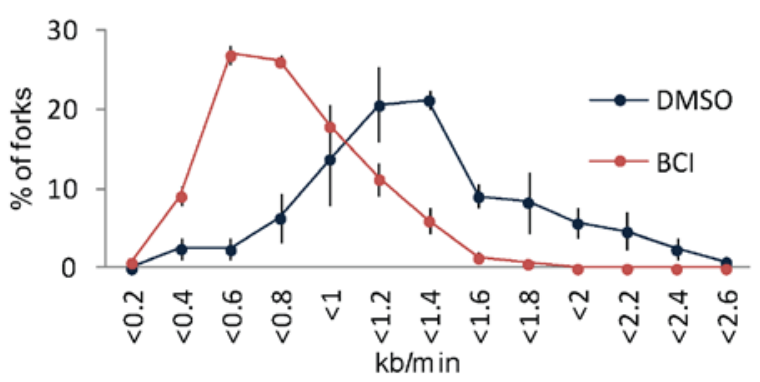

Distribution of total fork speed

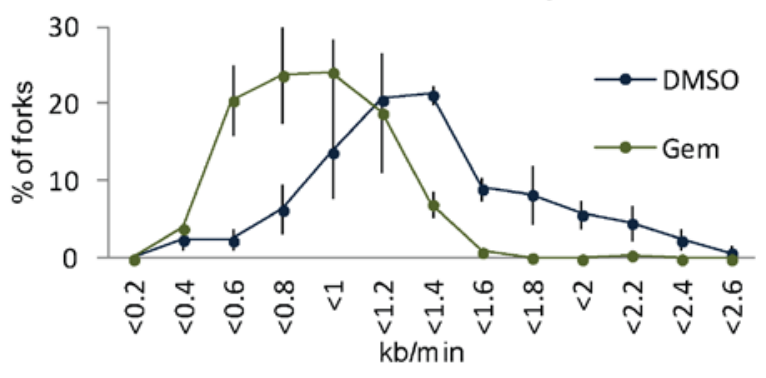

Distribution of total fork speed

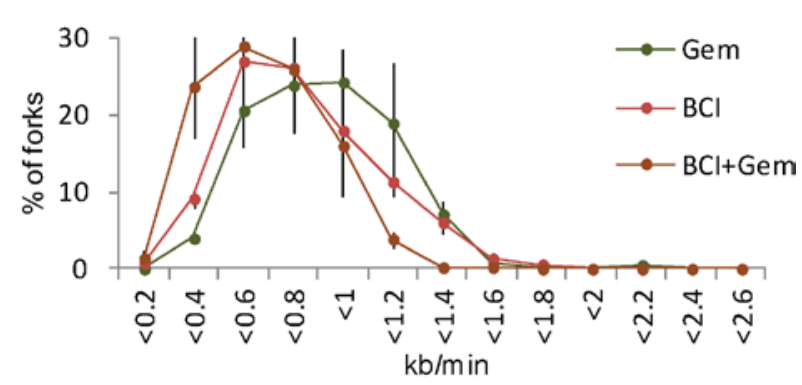

Figure 5.3 $\mathrm{BCl}$ and Gemcitabine co-operate to further impair the progression of replication fork.

(A) Labeling protocol for DNA fiber analysis. U2OS cells were pre-treated with $1 \mu \mathrm{M} \mathrm{BCl}$ or an equal volume of DMSO for $1 \mathrm{~h}$ and later labeled with CldU or IdU for 30 min each, in the presence of $\mathrm{BCl}$ or DMSO. $400 \mathrm{nM}$ gemcitabine was added along with IdU.

(B) Average of total replication fork speed (sum of CldU and IdU labeled track lengths) in cells treated as shown in (A) (n=3). Students t-test was used to evaluate significance of the data; $p$-values are displayed in the figures.

(C) Representative images of replication tracks after labeling according to A.

(D) Distribution of total replication fork speeds in the various conditions outlined above $(n=3)$. 


\subsection{Inhibition of MKP1 differentially regulates checkpoint signaling}

DNA replication is a tightly regulated process engaging various proteins, some of which are also a part of the DDR signaling cascade. To understand the dynamics of MKP1 in replication and DDR, (5.1 and 5.2); we looked for the activation of certain checkpoint kinases which are known for their interplay in the same; namely, MAPKAPK2 (MAPK Activated

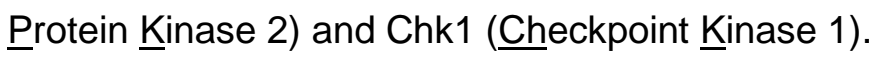

MK2 is a Ser/Thr kinase (also called Chk3), phosphorylated at Thr 344 and activated by p38MAPK. Originally known to feature in the inflammatory response, it was only recently discovered to operate alongside ATR-Chk1 and ATM-Chk2 in DDR. Most prominently, a study from Köpper et al., showed that gemcitabine induced DNA damage relies on MK2 activity to inhibit translesion synthesis. Chk1 is another canonical checkpoint kinase of the damage signaling axis closely associated with replication; whose inhibition decreases fork progression by promoting the firing of dormant origins (Petermann, Woodcock et al. 2010).

Immunoblot analysis from a time course experiment with DMSO / $\mathrm{BCl}$ done in the presence of a pan-caspase inhibitor Z-VAD-FMK [to block the activation of DDR by caspases (Matsuura, Wakasugi et al. 2008)] revealed the phosphorylation of p38MAPK as early as $1 \mathrm{~h}$ after exposure to $\mathrm{BCl}$. This was accompanied by the phosphorylation of Hsp27 (pHsp27 Ser 82), a downstream substrate of MK2, which was used to assess MK2 activity (Fig.5.4A, lanes $2,6,10)$. In contrast, Chk1 was not phosphorylated when cells were treated with $\mathrm{BCl}$ alone. This prompted us to check if there was any effect at all at the level of Chk1 activation. To that end, we used gemcitabine to induce Chk1 activation and subsequently monitored the phosphorylation status of Chk1. Remarkably; Chk1 was subjected to negative regulation by $\mathrm{BCl}$, in the presence of gemcitabine. This resulted in a loss of its phosphorylation (at Ser 317) and activity when both $\mathrm{BCl}$ and gemcitabine were combined (Fig. 5.4A, lanes 3 and 4; 7 and $8 ; 11$ and 12). We also checked for the activation of checkpoint kinase 2 (Chk2), which surprisingly, was also phosphorylated (pChk2 Thr 62) (Fig. 5.4B) in response to BCl. Unlike MK2 and Chk1, Chk2 has so far not been implicated in DNA replication, but is known to mediate cell cycle arrest during various stress conditions, including DNA damage. Collectively, this data indicates that MKP1, in addition to inactivating the stress response kinases - p38MAPK, JNK and ERK's (see 2.8), also modulates the activity of other protein kinases like Chk1 and Chk2, which are involved in replication and DNA damage response signaling. 
A

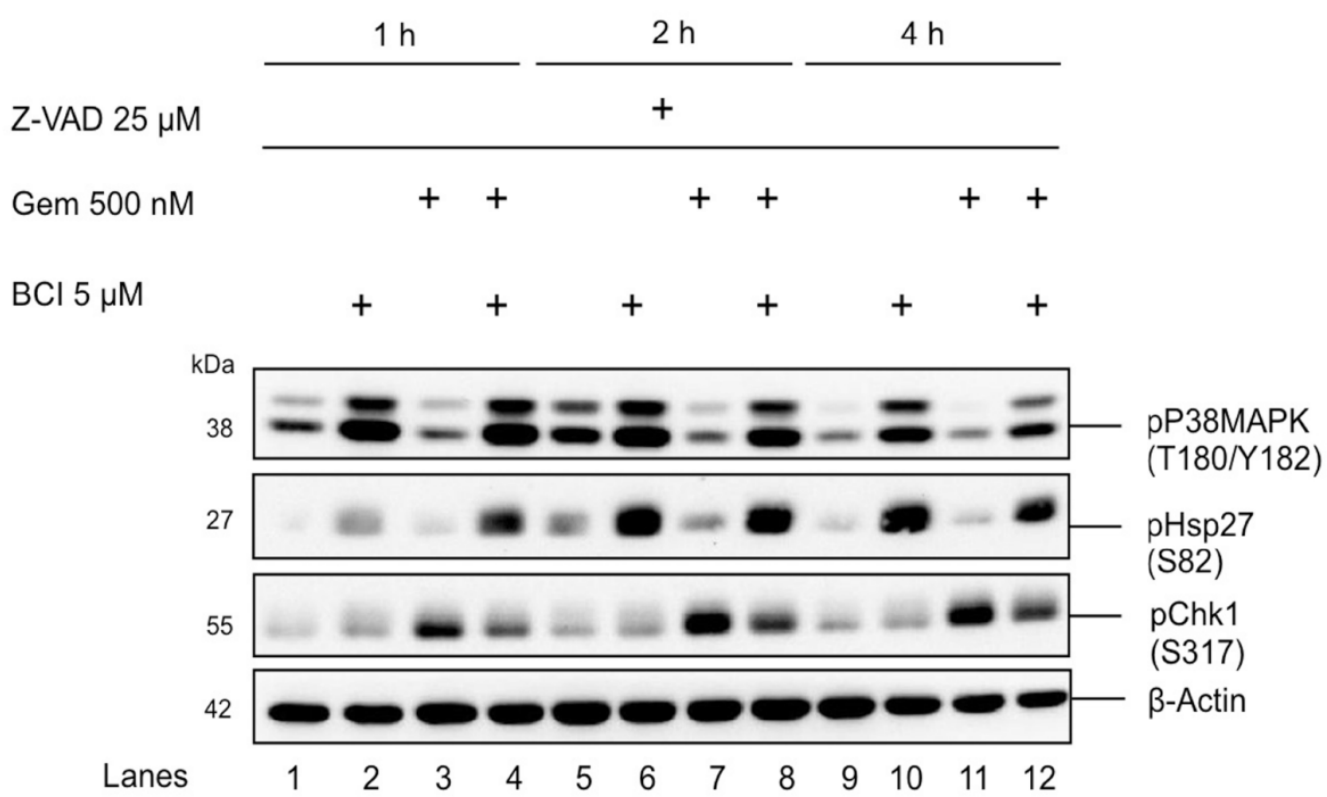

B

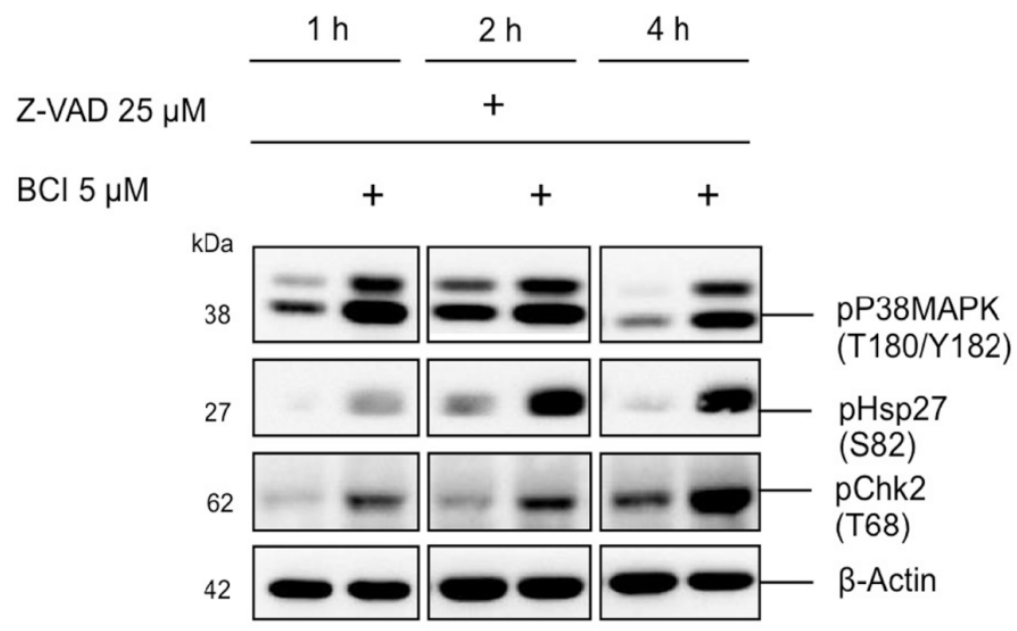

Figure 5.4 Activity of checkpoint kinases is subjected to differential regulation by MKP1.

(A) U2OS cells were treated with $5 \mu \mathrm{M} \mathrm{BCl}, 500 \mathrm{nM}$ Gemcitabine or an equal volume of DMSO in the presence of a pan-caspase inhibitor, Z-VAD-FMK $(25 \mu \mathrm{M})$.

(B) U2OS cells were treated with $5 \mu \mathrm{M} \mathrm{BCl}$ or an equal volume of DMSO in the presence of a pancaspase inhibitor, Z-VAD-FMK $(25 \mu \mathrm{M})$ to block apoptosis.

In both $(A)$ and $(B)$, protein extracts prepared after each of the indicated time points, were subjected to an immunoblot analysis with the antibodies mentioned in the figure. $\beta$-Actin served as a loading control. 


\subsection{Activation of ATM and MK2, is responsible for replicative stress induced by MKP1 inhibition}

Continuing our quest to delineate the pathways responsible for the disturbed replication seen with MKP1 inhibition, we again employed the DNA fiber assay, this time inhibiting the kinases - MK2 and Chk2, both of which were activated in the first hour of BCl treatment (see 5.4). MK2 was inhibited using a chemical competitive inhibitor - MK2III, and Chk2 activity was abolished by the inhibition of its upstream sensor kinase - Ataxia telangiectasia mutated (ATM). To look for any rescue effects, cells were pretreated with DMSO, MK2III or KU55933 (ATM inhibitor) for $30 \mathrm{~min}$ and later pulse-labeled with CldU and IdU in the presence of DMSO or BCl. MK2III / KU55933 / DMSO stayed on during the label as well (Fig. 5.5A and D). This was followed by cell lysis, and processed for DNA fiber spreads as described before. When MK2 was inhibited along with $\mathrm{BCl}$, there seemed to be no rescue in the average of total fork speed (Fig. 5.5E and F). However, it is possible that only certain populations of replication forks are rescued. This will be statistically insignificant when averaged over a large number of forks. Thus, we analyzed the distribution of fork speeds between $\mathrm{BCl}$ and $\mathrm{BCl}+\mathrm{MK} 2 \mathrm{III}$, where we found a slight difference between the two treatments (Fig. $5.5 \mathrm{H}$, page 56). Indeed, the percentage of forks with speeds between $1 \mathrm{~kb} / \mathrm{min}$ and $1.2 \mathrm{~kb} / \mathrm{min}$ was higher in the combination treatment as compared to the $\mathrm{BCl}$ treatment alone. Hence, MK2 rescues the slowly progressing forks, albeit very mildly.

Interestingly, the replication slow down caused by $\mathrm{BCl}$ was relieved by the simultaneous inhibition of ATM ( $p=0.01$ ) (Fig. 5.5B and C). This effect was also distinctly visible in the histograms depicting the distribution of total fork speeds (Fig. 5.5G, page 56). Complementing this finding was a decrease in the phosphorylation of $\mathrm{H} 2 \mathrm{AX}$ in an immunoblot experiment (Fig. 5.5I lanes 2 and 4; page 57), where cells were pre-incubated for 30 min with KU55933, followed by treatment with $\mathrm{BCl} / \mathrm{DMSO}$ in the presence of KU55933 for $4 \mathrm{~h}$. The slight phosphorylation of H2AX seen with DMSO could be a result of cellular stress induced by the same. Z-VAD-FMK was added to the all the samples for the same reason as outlined in 5.4. Here, phospho-Chk2 (Thr 62) was used as a read out for ATM activity, which clearly disappeared upon treatment with KU55933. Consequently, we hypothesized that an active MKP1 negatively regulates the p38MAPK/MK2 and ATM/Chk2 pathway to maintain the speed of elongating forks, adding a new dimension in the cascade linking phosphatases with DDR and replication. 
A

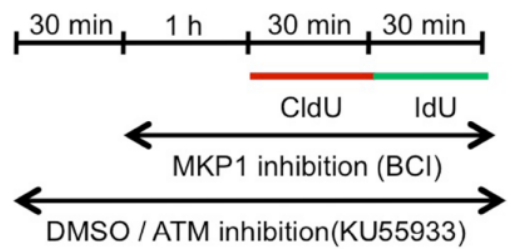

B

Average total fork speed

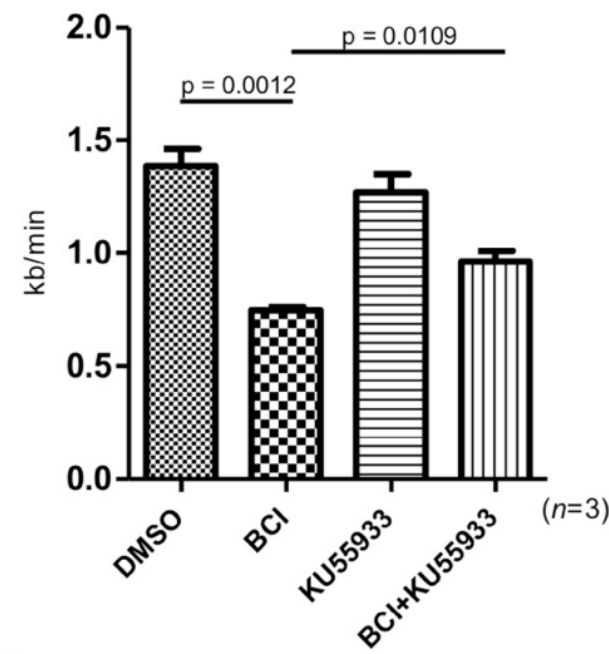

C

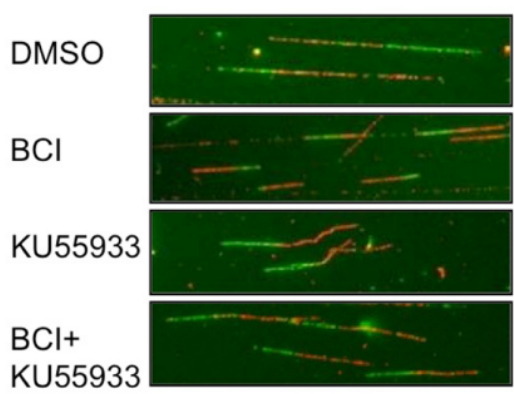

D

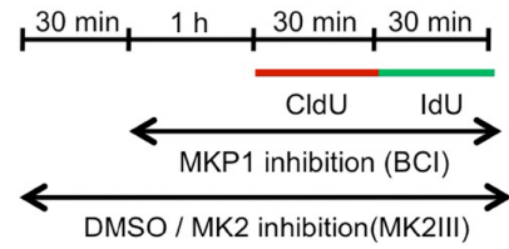

E

Average total fork speed

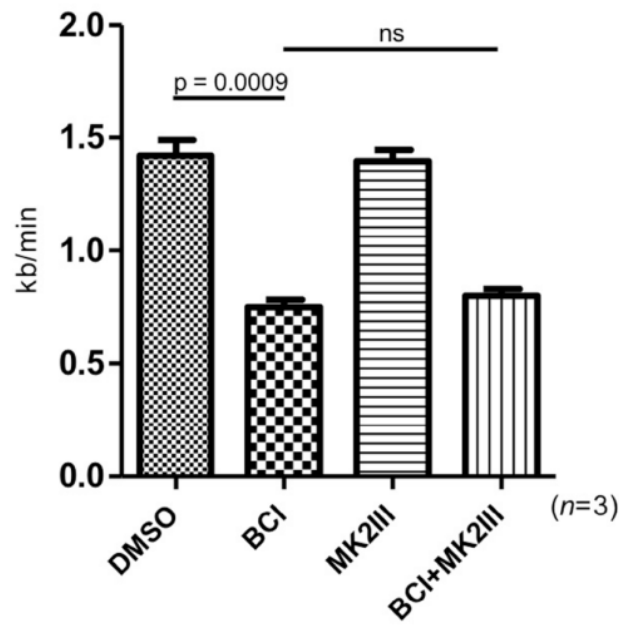

$\mathbf{F}$

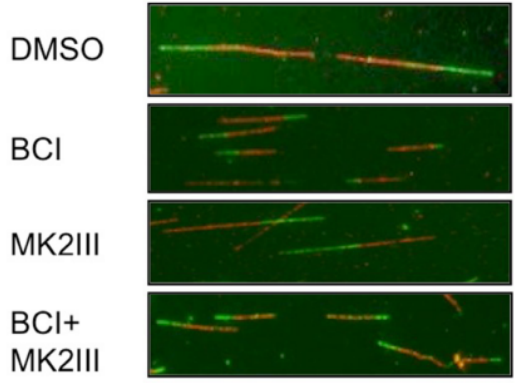

Figure 5.5 Replicative stress induced by MKP1 inhibition is dependent on ATM, but not MK2.

$(A, D)$ Labeling protocol for DNA fiber analysis. U2OS cells were first pre-treated with $10 \mu \mathrm{M}$ ATM inhibitor (KU55933) / an equal volume of DMSO in (A) or $10 \mu \mathrm{M}$ MK2 inhibitor (MK2III) / an equal volume of DMSO in (D) for $30 \mathrm{~min}$. This was followed by treatment with $3 \mu \mathrm{M}$ MKP1 inhibitor $(\mathrm{BCl})$ or an equal volume of DMSO for $1 \mathrm{~h}$ (both $\mathrm{A}$ and $\mathrm{D}$ ) in the presence of DMSO / KU55933 (in A) or DMSO I MK2III (in D). Later, cells received 30 min pulses of CldU and IdU in the continuous presence of the inhibitors as shown in (A) and (D).

$(B, E)$ Average of total replication fork speed (sum of CldU and IdU labeled track lengths) was calculated for cells treated as explained above $(n=3)$. Student t-test yielded $p$-values which are shown in the figures.

$(\mathrm{C}, \mathrm{F})$ Representative images of replication tracks. 
G

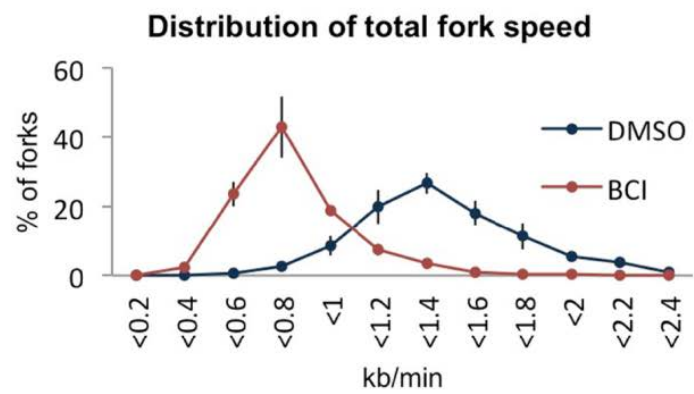

Distribution of total fork speed

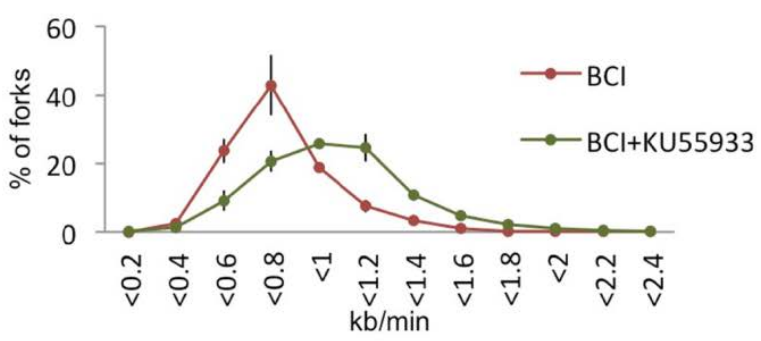

H

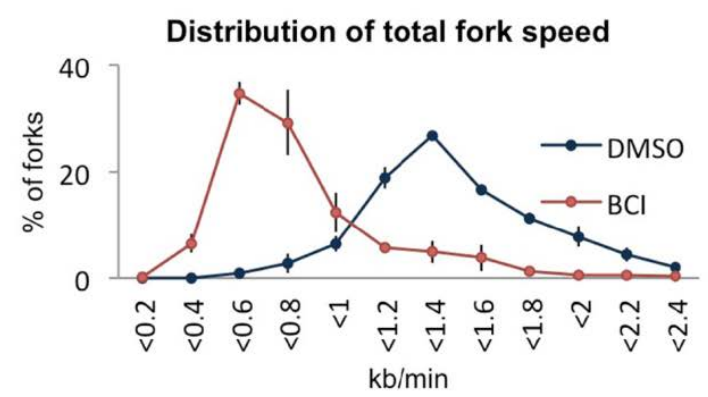

Distribution of total fork speed

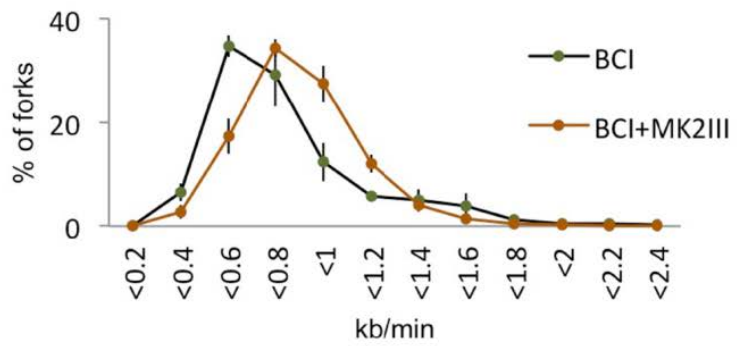

Figure 5.5 Continued.

$(G, H)$ Distribution of total replication fork speeds in the various conditions presented above $(n=3)$.

Carrying forward, we were determined to learn if the rescue in replication fork speed noticed with ATM inhibition was a result of a loss in ATM activity or Chk2 function. To this end, an immunoblot experiment was performed in a manner similar to the one outlined above, the only difference being the replacement of KU55933 with Chk2 inhibitor (Sc203885). Since pChk2 (Thr 68) accumulates in the presence of the Chk2 inhibitor Sc203885, this was used as a read out for Chk2 inhibitor efficiency (Sharma and Tepe 2004, Jobson, Lountos et al. 2009). As opposed to the results obtained with KU55933, the inhibition of Chk2 along with $\mathrm{BCl}$ did not affect the phosphorylation status of $\mathrm{H} 2 \mathrm{AX}$ (Fig. 5.5J, lanes 2 and 4). Using Chk2 inhibitor with $\mathrm{BCl}$ in DNA fiber experiments would give much clearer insights along this line, but based on these findings, we can still postulate that active ATM and not Chk2, contributes to the replication defects observed upon MKP1 inhibition (Figure on the next page). 
I

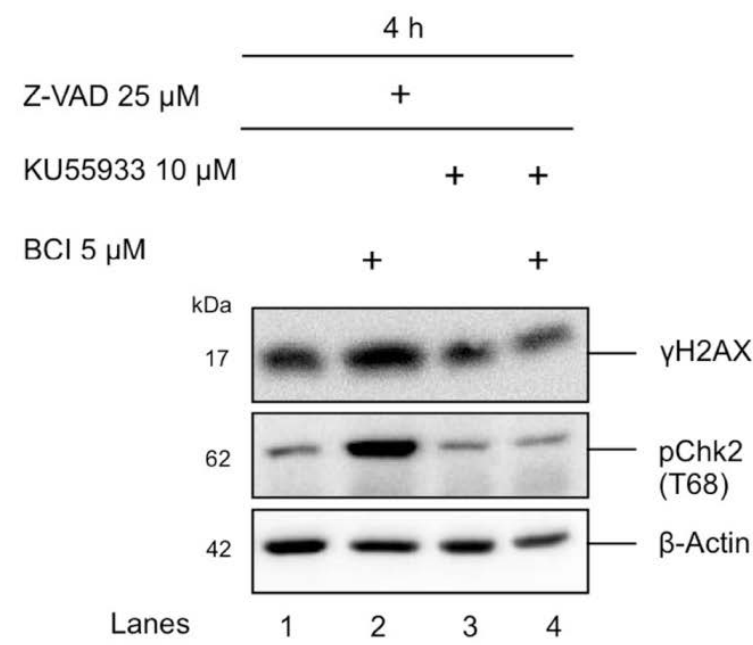

$\mathbf{J}$

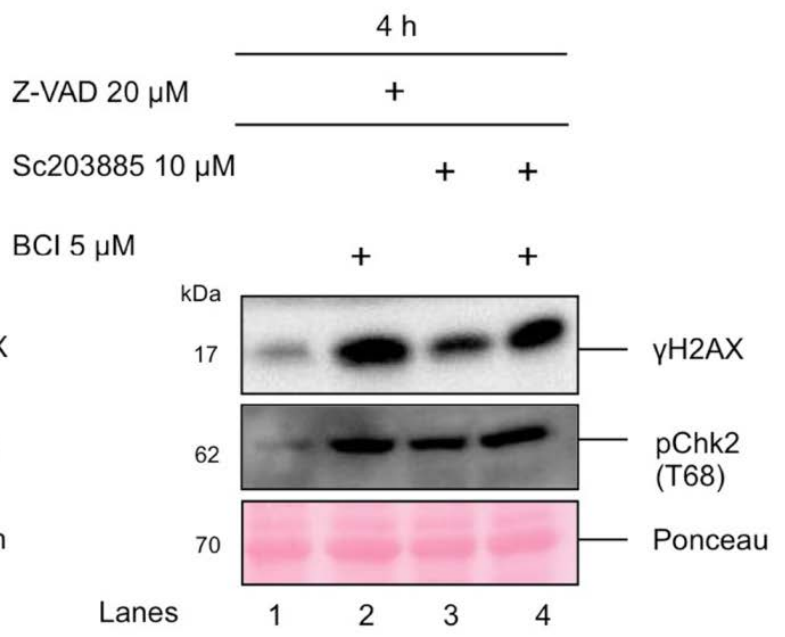

Figure 5.5 Continued.

Chk2 activity is not required for the effects of MKP1 inhibition.

(I) U2OS cells were exposed to $5 \mu \mathrm{M} \mathrm{BCl}$ with and without ATM inhibitor (KU55933) in the presence of Z-VAD-FMK for $4 \mathrm{~h}$, and harvested for an immunoblot analysis against the above specified proteins. $\beta$ Actin was used as a loading control.

(J) Experiment was performed as outlined in (I), the only difference being the replacement of KU55933 with Chk2 inhibitor Sc203885. Ponceau-S stain was used to check the efficiency of loading.

\subsection{MKP1 inhibition decreases origin firing, independently of ATM activity}

Replication fork speed and origin firing are inversely coupled processes. Under conditions of replicative stress, where fork progression is hampered, the dormant origins which are otherwise passively replicated by ongoing forks from their neighboring counterparts start firing, in order to compensate for the stalling and/or reduction in fork speed (see 2.5). So, besides analyzing the speed of replication upon MKP1 inhibition, we also estimated the level of origin firing by representing the first label origins (green-red-green) as a percentage of all red labeled structures (see 4.4). Strikingly, just like the rate of replication, the percentage of newly fired origins also showed a significant decrease with $\mathrm{BCl}$ treatment when compared to the DMSO control (Fig. 5.6A and C). Moreover, this effect remained unaltered even in the presence of KU55933; allowing us to suspect the existence of other MKP1 substrates controlling origin firing (Fig. 5.6B and D). 
A

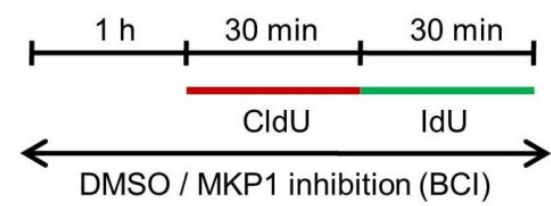

C Origin Firing

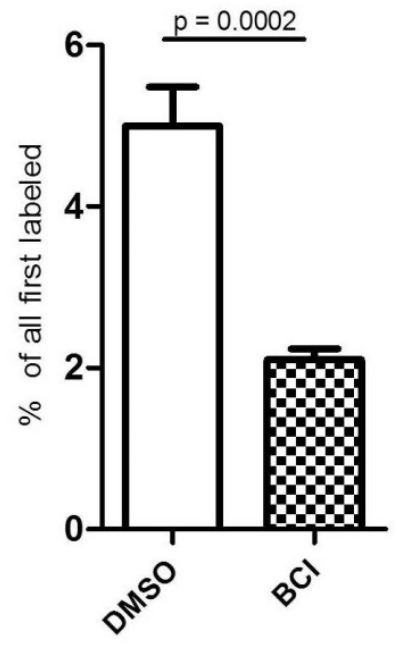

B

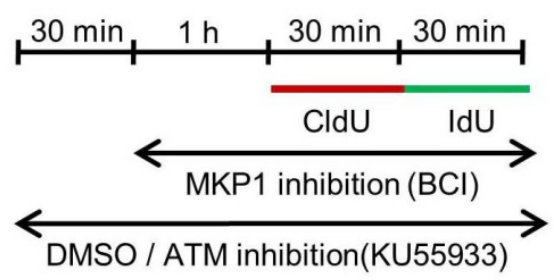

D Origin Firing

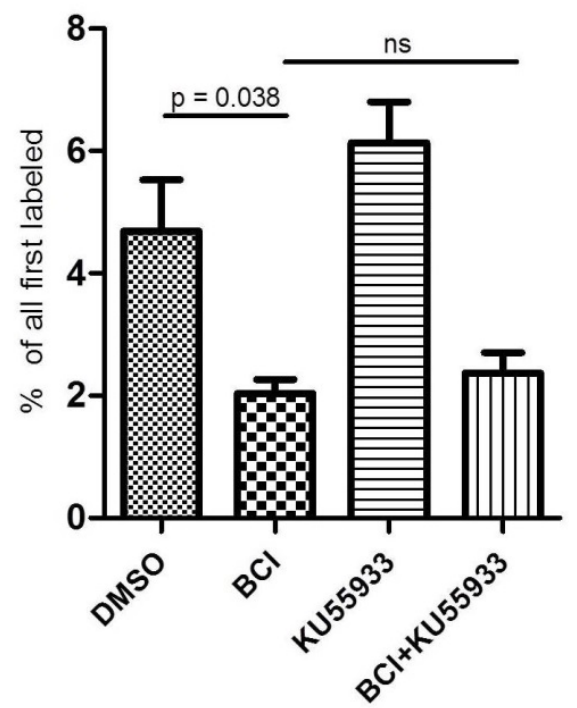

Figure 5.6 An ATM independent decrease in origin firing upon MKP1 inhibition.

(A) Labeling protocol for DNA fiber analysis. After pre-treatment with $3 \mu \mathrm{M} \mathrm{BCl}$ or DMSO for $1 \mathrm{~h}$, U2OS cells were kept in medium containing $\mathrm{CldU}+\mathrm{BCl} / \mathrm{DMSO}$ for $30 \mathrm{~min}$ and subsequently IdU+BCl I DMSO for another $30 \mathrm{~min}$. This was followed by cell lysis and immunostaining as described previously $(n=6)$.

(B) Labeling protocol for DNA fiber analysis. U2OS cells were first pre-treated with $10 \mu \mathrm{M}$ ATM inhibitor (KU55933) or an equal volume of DMSO for 30 min. Afterwards, $3 \mu \mathrm{M} \mathrm{MKP1} \mathrm{inhibitor} \mathrm{(BCl)} \mathrm{or}$ an equal volume of DMSO for $1 \mathrm{~h}$ was added to the cells in the presence of KU55933 or DMSO (wherever appropriate). Inhibitors stayed on the cells while they were received CldU and IdU pulses for 30 min each. Cell lysis and immunostaining was done as above $(n=3)$.

(C) and (D) First label origins (green-red-green, sample figure shown in methods) were counted and quantified as a percentage of all red labeled structures, for the cells treated as outlined above. Statistical analysis was done using students t-test, $p$-values are indicated in the figures. 


\subsection{Inhibiting MKP1 activity results in the loss of replication initiator kinase Cdc7 and MCM2 phosphorylation}

Origin firing is a spatio-temporally regulated event utilizing big-protein machineries, which

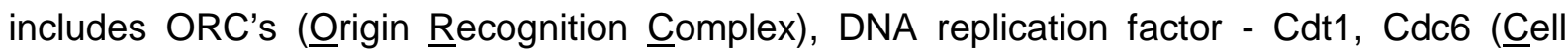
division cycle 6) and the ATP powered MCM2-7 complex (Mini-Chhromosome Maintenance). These proteins assemble sequentially at the origins to form a pre-replicative complex (pre$\mathrm{RC}$ ) during the G1 phase of cell cycle, but are still inactive. When cells enter S-phase, these proteins are phosphorylated by Cdk (ㄷyclin- $\underline{d} e p e n d e n t ~ k i n a s e)$ and DDK (ㅁbf4-Dependent Kinase), which then initiate replication (see 2.1.2).

DDK is a protein complex consisting of cell division cycle 7-related protein kinase ( $\mathrm{Cdc} 7)$ and its regulatory partner Dbf4. Cdc7 phosphorylates and activates MCM helicases that are required for triggering replication initiation by facilitating DNA unwinding. One such phosphorylation site is Ser 53 of MCM2, which is a direct target of Cdc7 kinase in the G1-S transition (Montagnoli, Valsasina et al. 2006) Considering this, we analyzed the kinetics of Cdc7 and MCM2 phosphorylation upon MKP1 inhibition, via immunoblotting the whole cell lysates prepared from $2 \mathrm{~h}$ and $4 \mathrm{~h} \mathrm{BCl} /$ DMSO treated cells. Remarkably, both Cdc7 levels and MCM2 phosphorylation disappeared upon $\mathrm{BCl}$ treatment (Fig. 5.7A) - a phenomenon which could explain the decreased rate of origin firing observed in 5.6.

Supplementing this finding is the work of Montagnoli et al., where PHA 767491, a well-known inhibitor of Cdc7 kinase, was shown to decrease the rate of origin firing without affecting the replication fork speed. Additionally, in our experiments we used two different inhibitors against Cdc7- PHA 767491 and XL413 (Montagnoli, Valsasina et al. 2008, Koltun, Tsuhako et al. 2012), and looked for the activation of certain proteins in the DNA damage and stress response pathways. $\mathrm{yH} 2 \mathrm{AX}$ was used as a read out for DNA damage whereas accumulation of pHsp27 was used for detection of stress response. Phospho-MCM2 Ser 53 was additionally stained to control for the potency of Cdc7 inhibitors. The immunoblot showed a gradual decrease in the phosphorylation of MCM2 over time, with the effects observable after $6 \mathrm{~h}$ of treatment, thus confirming Cdc7 inhibition (Fig. 5.7B-1 and B-2). In parallel, we could also detect the phosphorylation of $\mathrm{H} 2 \mathrm{AX}$ and Hsp27, very similar to the effects obtained with $\mathrm{BCl}$ treatment. It should be noted that with PHA767491 there is a loss of Cdc7 observed after $24 \mathrm{~h}$ of treatment. This is an unexplained phenomenon also seen by Montagnoli et al. Although pChk2 was not checked, nonetheless, it will be very interesting to look into its activation pattern as well. In totality, these observations point towards a possible link between MKP1 and Cdc7 to regulate replication initiation. 
A

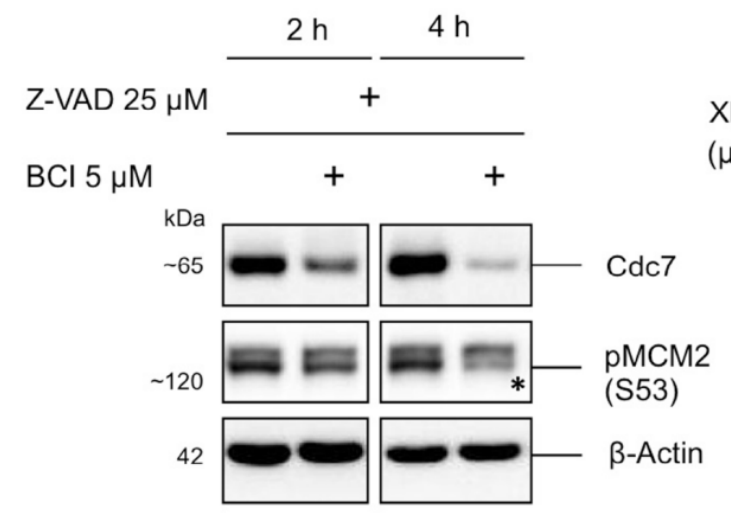

B-1

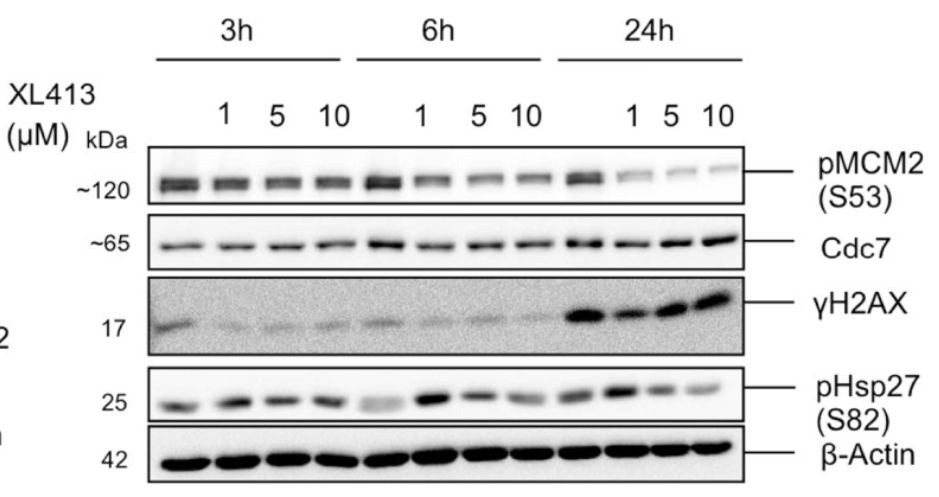

B-2

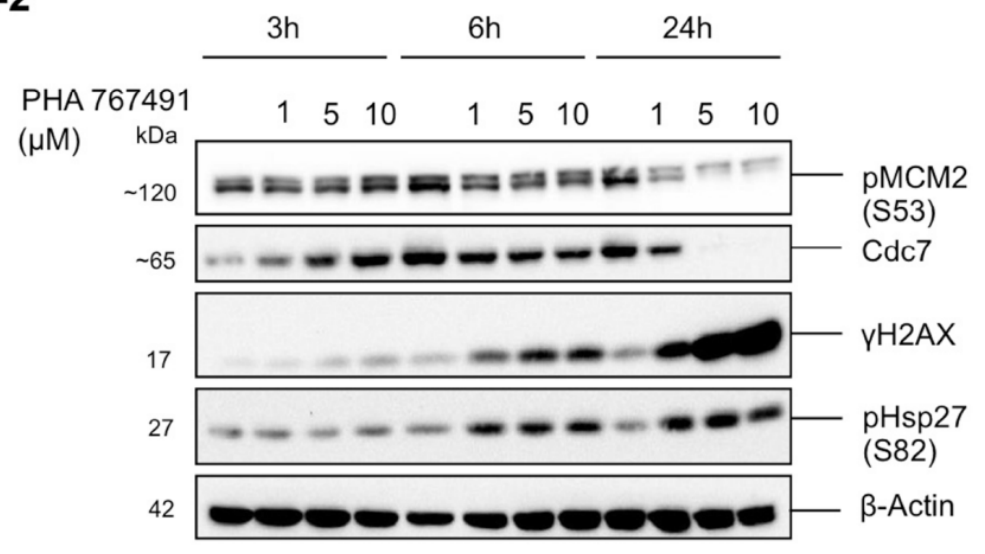

Figure 5.7 MKP1 inhibition leads to a decrease in Cdc7 levels and MCM2 phosphorylation.

(A) U2OS cells were incubated with $5 \mu \mathrm{M} \mathrm{BCl}$ or an equal volume of DMSO for $2 \mathrm{~h}$ and $4 \mathrm{~h}$, in the presence of Z-VAD-FMK. Cells were harvested after each time point, and protein extracts were analyzed by immunoblotting using the indicated antibodies. $\beta$-Actin was used as a loading control. Asterix $\left({ }^{\star}\right)$ indicates the protein of interest.

(B) U2OS cells were treated with 1, 5 and $10 \mu \mathrm{M}$ Cdc7 inhibitors, XL413 (B-1) or PHA767491 (B-2) for the indicated time points and harvested for immunoblot. An equal volume of DMSO corresponding to $10 \mu \mathrm{M}$ of the inhibitor was used as a control. Proteins were detected using the indicated antibodies. $\beta$ Actin was used as a loading control. 


\subsection{Proteasome degradation or mRNA regulation are not responsible for the loss of Cdc7 after MKP1 inhibition}

Decreased protein levels are mainly due to a loss in mRNA copies or degradation of the protein itself. To test which out of the two is responsible for the apparent reduction of $\mathrm{Cdc} 7$ levels seen with MKP1 inhibition (see 5.7), cells were treated with DMSO or $\mathrm{BCl}$ in the presence of Z-VAD-FMK, for $1 \mathrm{~h}, 2 \mathrm{~h}$ and $4 \mathrm{~h}$; RNA was isolated and a quantitative RT-PCR was done using primers specific to Cdc7 (experiment conducted with Christin Kellner). This allowed us to measure the Cdc7 mRNA levels, which remained unchanged with $\mathrm{BCl}$ treatment (Fig. 5.8A). Thereupon, we exposed the cells to a proteasome inhibitor - MG132 along with $\mathrm{DMSO} / \mathrm{BCl}$ and analyzed total protein levels. To our surprise, this as well did not aid in recovering the lost Cdc7 (Fig. 5.8B, lanes 2 and 4; 6 and 8).

As there are additional levels of regulation at the step of translation, where mRNA's can be modified to block protein synthesis, and post-translation, where proteins are modified by the addition of small groups (phosphate, ubiquitin, SUMO etc.) that can mask its epitope required for antibody recognition; this ambiguous loss of $\mathrm{Cdc} 7$ could be a result of such a modification and thus still be explored further.

Co-incidentally when we examined Cdc7 levels in the $\mathrm{BCl}$ and ATM inhibited samples, it still remained lost (Fig. 5.8C, lanes 2 and $4 ; 6$ and 8). This was in accordance with our DNA fiber data where origin firing was unaffected in the presence of KU55933 (see 5.6).

Summarizing the results above, we can state that MKP1 inhibition reduces the replication fork rate by inactivating the two kinases, MK2 and ATM. Additionally, Cdc7 is also downregulated, which may explain the reduced origin firing seen when MKP1 is blocked. 

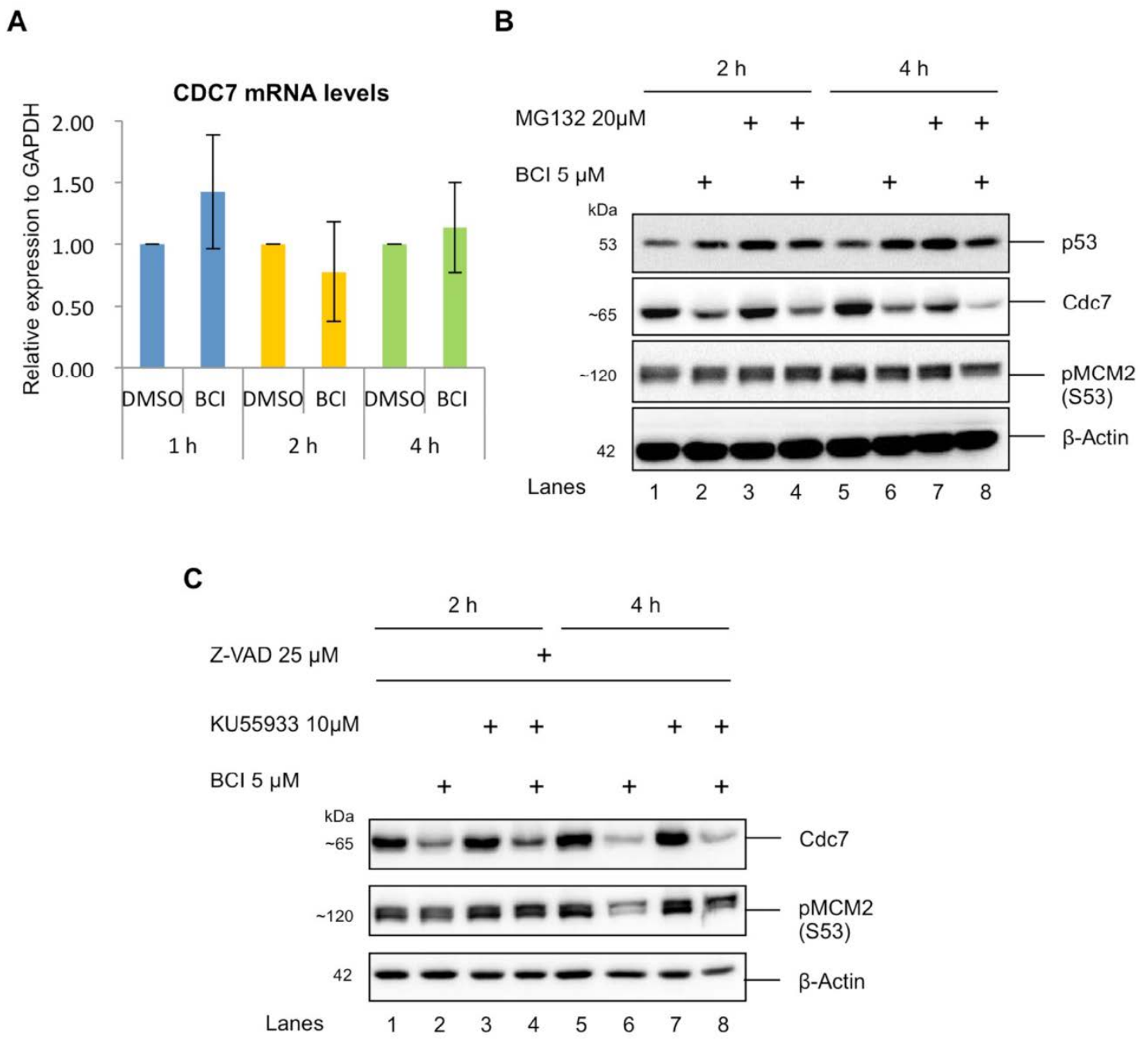

Figure 5.8 Disappearance of Cdc7 upon MKP1 inhibition is not due to a loss of mRNA or protein.

(A) U2OS cells were treated with $5 \mu \mathrm{M} \mathrm{BCl}$ or an equal volume of DMSO for $1 \mathrm{~h}, 2 \mathrm{~h}$ and $4 \mathrm{~h}$ in the presence of Z-VAD-FMK. After each time point, total RNA was extracted using TRIzol and gene expression of Cdc7 was analyzed via qRT-PCR. GAPDH was used as the reference gene for normalization.

(B) U2OS cells were treated with $5 \mu \mathrm{M} \mathrm{BCl}$ or an equal volume of DMSO in the presence of $20 \mu \mathrm{M}$ proteasome inhibitor - MG132 for $2 \mathrm{~h}$ and $4 \mathrm{~h}$.

(C) U2OS cells were treated with $5 \mu \mathrm{M} \mathrm{BCl}$ and $10 \mu \mathrm{M}$ ATM inhibitor KU55933 for the indicated time points. In both (B) and (C) cells were subsequently harvested for protein and analyzed by immunoblot using the antibodies specified in the figure. 


\subsection{Longer exposure to $\mathrm{BCl}$ or reduction of MKP1 levels leads to apoptosis}

It is a well-known fact that prolonged replicative stress can result in collapsed replication forks thereby setting off the alarms for apoptosis - a programmed set of signaling events brought about by the action of certain specific proteases, called caspases, culminating in cell death. To shed some light on this in the context of MKP1 inhibition; we did a simple immunoblot after treating cells with $\mathrm{BCl}$ (or DMSO as control) for longer hours and stained for the apoptotic markers - caspase-3 and PARP-1. Caspase-3 is a cysteine-aspartic protease existing in an inactive zymogen state in the absence of apoptotic stimuli. Upon receiving the appropriate signal, caspase- 3 is cleaved into its active form, which is then responsible for chromatin condensation and DNA fragmentation that accompanies the morphological changes seen during apoptosis. On the other hand, PARP-1 (Poly [ADPribose] polymerase 1 ) is a DNA repair protein that carries out its function by modifying its acceptor by Poly-ADP-ribosylating it. This also undergoes cleavage and hence inactivation at the time of apoptosis. As predicted, $12 \mathrm{~h}$ of $\mathrm{BCl}$ treatment led to the activation of caspase-3 and inactivation of PARP-1, proving that extended MKP1 inhibition can indeed be cytotoxic (Fig. 5.9A). Furthermore, identical results were obtained with a transient depletion of MKP1 from the cells using siRNA mediated knockdown approach, thus re-affirming our inhibitor data (Fig. 5.9B).

A

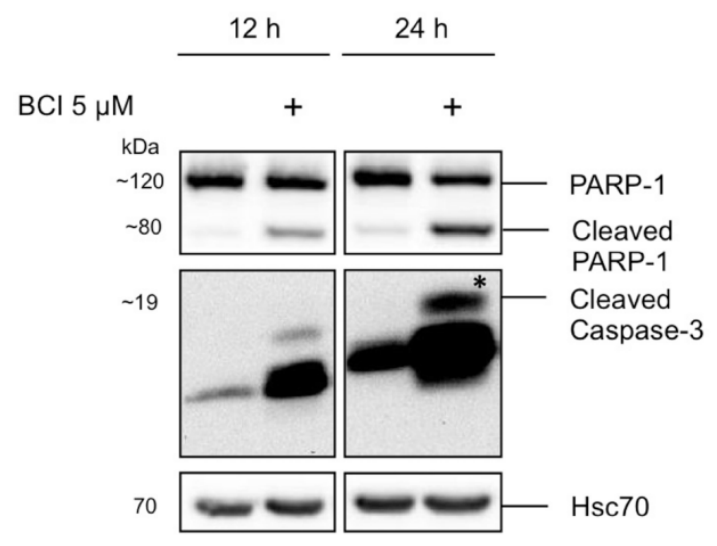

B

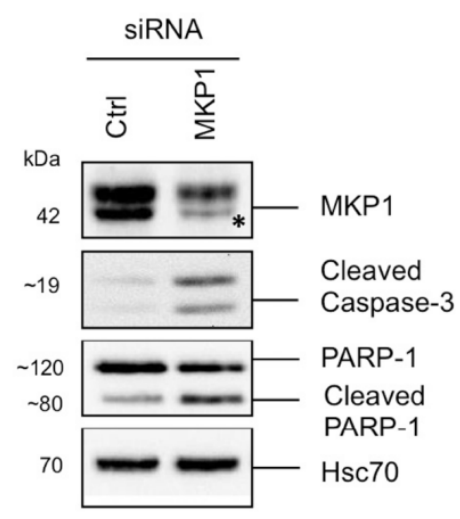

Figure 5.9 MKP1 knockdown or $\mathrm{BCl}$ treatment for long hours leads to apoptosis.

(A) U2OS cells were treated with $5 \mu \mathrm{M} \mathrm{BCl} 12 \mathrm{~h}$ and $24 \mathrm{~h}$ and harvested after each time point. Whole cell lysates were subjected to an immunoblot assay and proteins shown in the figure were detected using corresponding antibodies. Asterix $\left(^{\star}\right)$ indicates the protein of interest.

(B) U2OS cells were reverse transfected with a non-targeting siRNA or siRNA specific to MKP1 (designated as s4363, $10 \mathrm{nM}$ ) using lipofectamine and harvested after $48 \mathrm{~h}$ for immunoblot analysis. Asterix $\left(^{*}\right)$ indicates the protein of interest. Hsc70 was used as a loading control in both (A) and (B). 


\subsection{MKP1 knockdown is accompanied by a caspase independent degradation of an anti-apoptotic protein, Mcl-1}

While replicative stress can be one reason for apoptosis observed with MKP1 inhibition/depletion, we were eager to find out if this was the only culprit. To reflect upon this, we used a candidate approach to look for any apoptotic protein that might be regulated by MKP1. For this and all following experiments, we carried out transient knockdown of MKP1 as it ensured complete depletion of the protein and at the same fit the longer time frame criteria for observing apoptosis. The protein lysates were subsequently analyzed via immunoblot for various pro-apoptotic as well as anti-apoptotic proteins (data not shown). A non-targeting siRNA was used as a control. One interesting candidate was an anti-apoptotic protein Mcl-1 (Myeloid cell leukemia 1); which became nearly undetectable upon MKP1 knockdown (Fig. 5.10A, lanes 1 and 2). 350 amino acids long, this protein belongs to the Bcl2 family of pro / anti-apoptotic regulators and is localized to the mitochondria where it binds to and inactivates the pro-apoptotic factors, e.g. Bim and Bax (see 2.5).

Since Mcl-1 is also subjected to cleavage by caspases, its disappearance observed with MKP1 depletion, could be a manifestation of apoptosis rather than the cause itself (Herrant, Jacquel et al. 2004, Weng, Li et al. 2005, Hu, Dang et al. 2011). Therefore, we performed a knockdown for $48 \mathrm{~h}$ this time in the presence of a pan-caspase inhibitor, Z-VAD-FMK. This however did not influence our results, and the loss of Mcl-1 still remained demonstrating that Mcl-1 degradation is not a consequence of apoptosis in this context (Fig. 5.10A, lanes 3 and 4).

These findings motivated us to study the mechanics of Mcl-1 regulation, and in this reference we checked if the loss of Mcl-1 was dependent on the proteasome machinery (Nijhawan, Fang et al. 2003, Derouet, Thomas et al. 2004). Therefore, the knockdown experiments were performed along with a proteasome inhibitor - MG132, which was added in the last 4 hours of transfection. This approach rescued the lost levels of Mcl-1 (Fig. 5.10B) and led us to believe that MKP1 might act to protect Mcl-1 from degradation, thereby maintaining a fine balance between apoptosis and survival. 
A

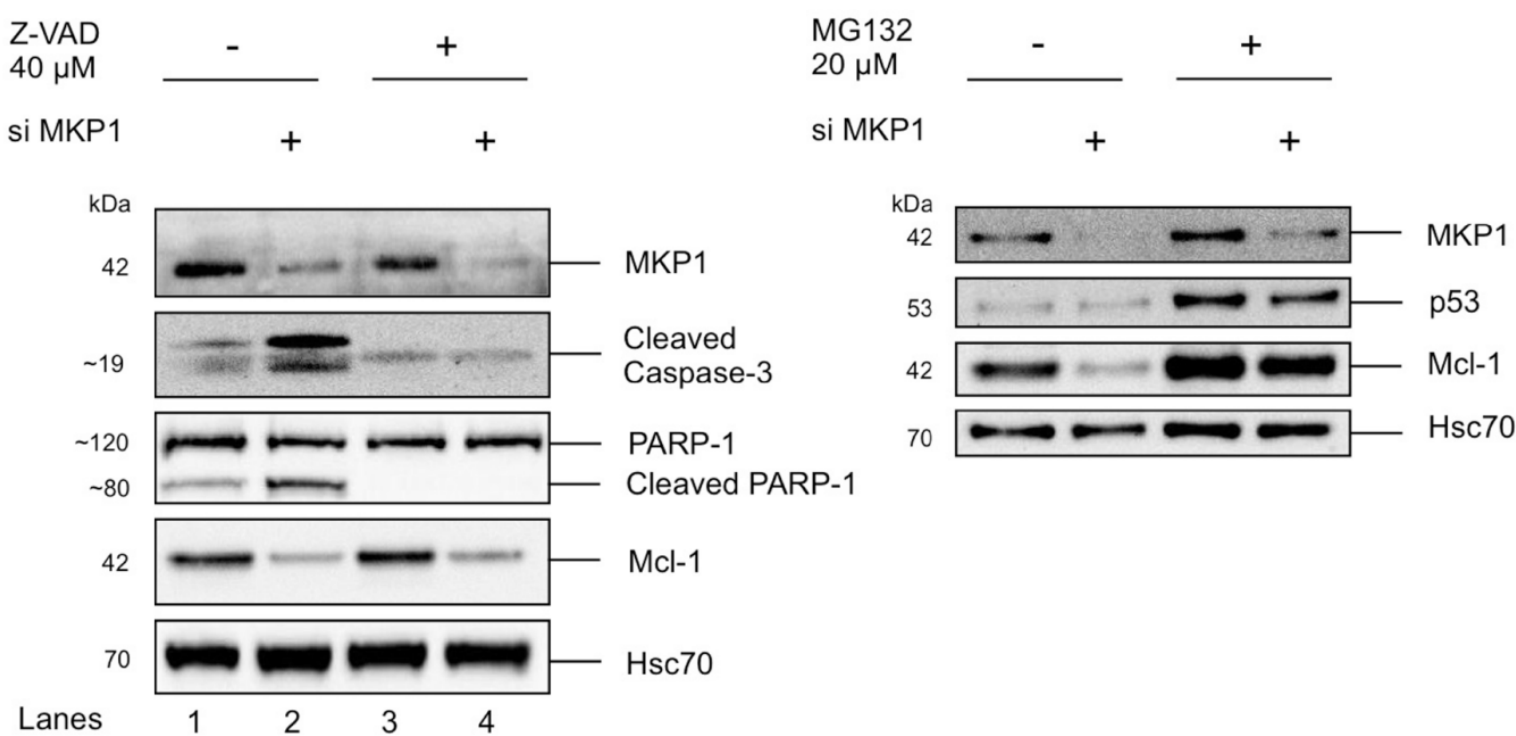

B

Figure 5.10 Anti-apoptotic protein Mcl-1 is degraded independent of caspase activity upon MKP1 depletion.

(A) U2OS cells were reverse transfected with $10 \mathrm{nM}$ MKP1 or non-targeting siRNA using lipofectamine. $24 \mathrm{~h}$ after transfection, a pan-caspase inhibitor Z-VAD-FMK was added, and left on for an additional $24 \mathrm{~h}$.

(B) U2OS cells were reverse transfected with $10 \mathrm{nM}$ MKP1 siRNA for $48 \mathrm{~h}$. A non-targeting siRNA was used as a control. Proteasome inhibitor, MG132 $(20 \mu \mathrm{M})$, was added in the last 4 hours of transfection. After treatment according to $(A)$ or (B) cells were harvested for proteins and immunoblot analysis was performed. 


\subsection{Apoptosis upon MKP1 depletion can be partially rescued by Mcl-1 over-expression}

To confirm whether Mcl-1 degradation was indeed specifically responsible for cell death upon MKP1 knockdown, we over expressed Mcl-1 to see if this would rescue the detected phenotype. Cells were first reverse transfected with non-targeting or MKP1 siRNA and $24 \mathrm{~h}$ later, forward transfected with an empty or Mcl-1 over-expressing plasmid for an additional $24 \mathrm{~h}$. This was followed by protein extract preparation and immunoblot analysis.

As shown in Fig. 5.11 (lanes 3 and 4), Mcl-1 over-expression was detectable as expected. Bringing back the levels of Mcl-1 proved beneficial for the cells, as now the cells lacking MKP1 had much lower levels of cleaved PARP-1 (Fig. 5.11, lanes 2 and 4). Although restoring Mcl-1 did not completely help in cellular survival; one should not rule out the existence of replicative stress, which can still contribute to apoptosis in cells deprived of MKP1.

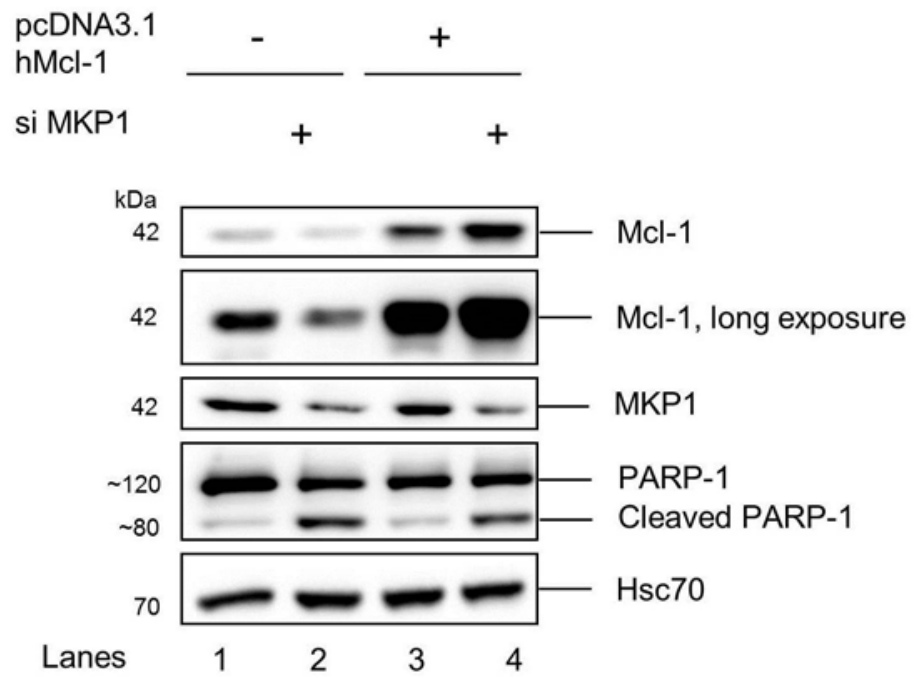

Figure 5.11 Apoptotic effects of MKP1 knockdown are partially rescued by over-expression of Mcl-1.

U2OS cells were reverse transfected with $10 \mathrm{nM}$ non-targeting or MKP1 siRNA using lipofectamine. 24 $\mathrm{h}$ after transfection, cells were re-transfected with a Mcl-1 over-expression vector- pcDNA3.1 hMcl-1 (375 $\mathrm{nG}$ ) or pcDNA3 empty vector for $4 \mathrm{~h}$. After this media was changed and cells were allowed to grow in full medium for an additional $20 \mathrm{~h}$ and later harvested for an immunoblot analysis. Hsc70 was used as a loading control. 


\subsection{MKP1 interacts with Mcl-1 under unstressed conditions}

Nuclear MKP1 regulates the activity of mitochondrial Mcl-1. How is this possible, unless they come in close proximity to each other? To answer this question, we took the classical approach of using in vivo complex-immunoprecipitation to look for an interaction between these proteins. U2OS were transfected with a plasmid encoding the cDNA of myc-DDK tagged MKP1 or FLAG-Mcl-1 (Morel, Carlson et al. 2009). When we pulled down Mcl-1 in the cell lysate using the anti-Mcl-1 antibody, we precipitated MKP1 as well in those samples which were co-transfected with both the plasmids (Fig. 5.12). This was not the case when cells were transfected with MKP1 vector alone. This interaction was further re-enforced by a reverse IP, where MKP1 was pulled down using an anti-myc antibody in the cell lysate and Mcl-1 was co-immunoprecipitated in a similar experimental setting as described above (experiment conducted with Christin Kellner). Even though this is not on an endogenous level, the presence of an interaction itself, strongly suggested that Mcl-1 is subjected to direct regulation by MKP1.
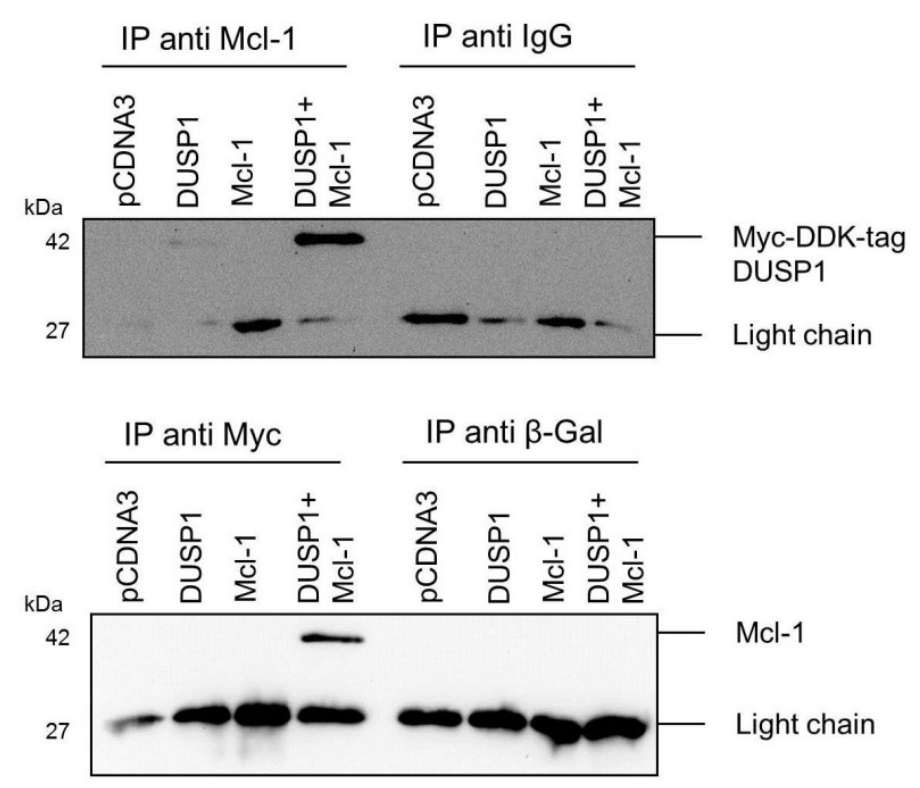

Figure 5.12 MKP1 interacts with Mcl-1 in an in-vitro co-immunoprecipitation assay.

U2OS cells were transfected for $24 \mathrm{~h}$ with pcDNA3 empty, pCMV-Myc-DDK-hDUSP1 (alias MKP1), and pCMV-Flag-Mcl-1 over-expressing plasmids, using lipofectamine 2000, as indicated in the figures. Cells were harvested for Co-IP and pull down was done with the antibodies indicated in the figures. (Top) Pull down was done with Mcl-1 antibody and IgG was used a control. (Bottom) Pull down was done with Myc antibody and $\beta-G a l$ was used as a control in this case. After the pull down and several washing steps later, the protein lysates were subjected to an SDS-PAGE, followed by immunoblotting. Blots were probed with the antibodies as indicated in the figures. 


\section{Discussion}

DNA is the storehouse of genetic information, with its sequence dictating the basis of all life. This makes it essential for cells to protect their DNA from a wide range of exogenous and endogenous genotoxic insults. Cellular DNA damage response tries to achieve this by activating a highly coordinated set of signaling events, which ensures genomic stability and facilitates DNA repair. This prevents DNA mutations that could result in uncontrolled cellular proliferation and tumor formation. DDR has long been known as a kinase dominated network, and only the last decade of work has witnessed a role for phosphatases, emerging in the same.

To further explore this area of research, we investigated the contribution of a dual-specificity phosphatase, MKP1, in DDR. We found that a loss of MKP1 activity is sufficient to accumulate phospho H2AX in various cancer cells lines. Importantly, this was seen in the absence of any exogenous DNA damaging agent. Thus, we speculated a defect in replication kinetics to be responsible for this kind of spontaneous DNA damage observed with MKP1 inhibition. Indeed, replication fork speed and origin firing were significantly reduced in the absence of MKP1. Additionally, MKP1 inhibition further decreased the replication fork rate in the presence of a nucleoside analog, gemcitabine. Furthermore, the impaired fork progression caused by MKP1 inhibition was dependent on the activity of the replication checkpoint kinases, MK2 and ATM. While MK2 is known to inhibit replication by inhibiting translesion synthesis, the role of active ATM in promoting replicative stress has not yet been described. Besides this, MKP1 inhibition was also accompanied with the loss of $\mathrm{Cdc7}$ kinase, a replication initiator protein which is required for origin firing. In addition to its effects on DNA replication, MKP1 is also essential for cellular survival. MKP1 interacts with an anti-apoptotic protein, Mcl-1, and protects it from getting degraded. This might be required for maintaining a fine balance between pro-survival and pro-apoptotic proteins, to avoid cell death in the absence of stress stimuli.

Based on our studies, we have identified that MKP1 is necessary for promoting DNA replication both in the presence and absence of external stress stimuli. This may be essential to prevent replicative stress arising from the accumulation of irreparable, spontaneously stalled replication forks. Since certain phosphatases like PP2A (Protein Phosphatase 2A) and PP1 (Protein Phosphatase 1) are known to regulate DNA replication, the involvement of MKP1 in the same seems conceivable (Lin, Walter et al. 1998, Petersen, Chou et al. 2006, Dave, Cooley et al. 2014). Furthermore, many previous findings have only reported the inactivation of JNK by MKP1, to be responsible for preventing cell death (Sanchez-Perez, Martinez-Gomariz et al. 2000, Chattopadhyay, Machado-Pinilla et al. 2006, Takeuchi, Shin- 
ya et al. 2009). With our data, we demonstrate a direct regulation of the Bcl-2 family member, Mcl-1, upon MKP1 inhibition (Fig. 6.1).

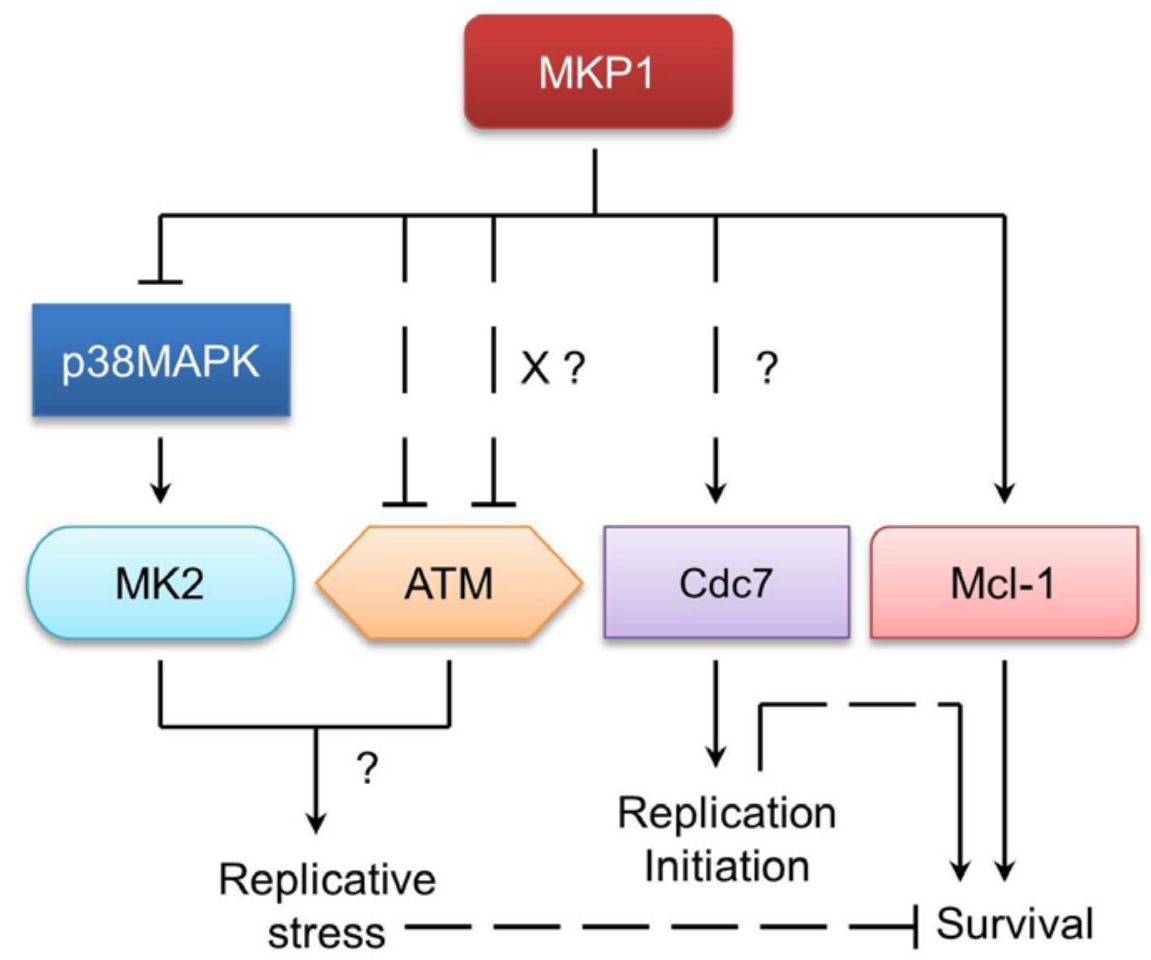

Figure 6.1 A hypothetical model representing the role of MKP1 in replicative stress and cell survival.

MKP1 inhibits the p38MAPK/MK2 pathway and the kinase ATM, to maintain normal replication fork rates and avoid replicative stress. Additionally, MKP1 also maintains stable levels of Cdc7 and promotes replication initiation. Besides this, Mcl-1, an anti apoptotic protein is also protected from proteasomal degradation by interaction with MKP1, promoting cellular survival.

$\longrightarrow$ Activation

$\longrightarrow$ Inhibition

Solid lines indicate a direct regulation and dashed lines indicate a possible direct regulation.

While the effects of MKP1 activity loss are very prominent, the mechanisms are not so clear. Does MKP1 directly regulate the activity of ATM? Or is it because of some intermediate factors? How are Cdc7 and Mcl-1 protected by MKP1 from being lost? How does MK2 participate in this context? These are some open questions that arise from our findings, which we will discuss in the subsequent sections.

\subsection{MK2 in controlling replication events upon MKP1 inhibition}

In our studies, MKP1 inhibition impaired replication fork progression and reduced the percentage of origin firing significantly (see 5.2 and 5.6). This was accompanied by the activation of MKP1 substrate, p38MAPK, which in turn phosphorylated its downstream target, MK2. Previous research has shown that gemcitabine-induced replication fork slowing relies 
on the activity of MK2 to inhibit TLS polymerases (Kopper, Bierwirth et al. 2013). This motivated us to check if activated MK2 was responsible for the replication defects observed with MKP1 inhibition, as well. Strikingly, the hindered fork progression in our system seemed to be only partially dependent on MK2 activity (see 5.5). However, it should be noted that the changes observed here using fiber assays, were based on short term treatments of approximately 1-2 $\mathrm{h}$. Thus, it is possible that during such a short time, TLS is only partly inactivated perhaps by a post translational modification; that does not generate very profound effects on replication (Köpper 2013). This technical detail gave us room to speculate the existence of a larger contribution from MK2, perhaps at the later stages of checkpoint activation. Indeed, preliminary immunoblot analysis performed with simultaneous inhibition of MK2 and MKP1 for $24 \mathrm{~h}$, partially rescued the accumulation of MKP1 inhibitioninduced, DNA damage ( $\mathrm{yH} 2 \mathrm{AX}$ ) (Appendix, Fig. 7.1, lanes 2 and 4). This result suggests that MK2 might as well be functional in DNA replication as a late-checkpoint kinase. Still, we need to confirm this hypothesis by conducting DNA fiber experiments with longer periods of inhibitor treatments (e.g. 24 h). Nevertheless, supporting this argument is the work from Reinhardt and group, who have previously reported such a late activation of MK2 for the maintenance of a prolonged DNA damage checkpoint (Reinhardt, Hasskamp et al. 2010).

Additionally, MK2 is known to phosphorylate and inactivate the Cdc25 phosphatases, which are positive regulators of cyclin-Cdk2 complexes. As active cyclin-Cdk complexes are required to initiate origin firing by phosphorylating MCMs (see 2.1.2), such an activation of MK2 can explain the reduced origin firing seen upon MKP1 inhibition. Thus, monitoring origin firing in DNA fiber assays would provide us with more insights on this aspect. Moreover, examining the status of Cdc25A and Cdk2 (Cdk2pTyr15) in immunoblots, will provide us with answers for the molecular basis of the same. In conclusion, based on our results, we can postulate that MKP1 inhibition activates MK2 that induces a mild replicative stress, perhaps by inhibiting TLS polymerases.

\subsection{ATM: Deciphering the code in replication fork progression}

If the reduced fork rate upon MKP1 inhibition is not completely dependent on MK2; then what are the other reasons for observing such an effect? While seeking an answer to this question, we identified the kinase ATM, whose activation was also responsible for mediating the effects we observed (see 5.5). This result immediately posed two new questions, firstly, how would MKP1 inhibition promote ATM activity? And secondly how does ATM act to inhibit fork progression?

While protein phosphatases Wip1 and PP2A are known to inactivate ATM, MKP1 has not been reported so far to dephosphorylate this kinase (Goodarzi, Jonnalagadda et al. 2004, 
Shreeram, Demidov et al. 2006, Goodarzi, Douglas et al. 2007). Besides, being a Thr/Tyr specific phosphatase, the likelihood of MKP1 directly dephosphorylating ATM might be slim as this kinase is phosphorylated at Ser residues during activation (Ser 1981, Ser 1893 and Ser 367) (Kozlov, Graham et al. 2011). However, we cannot completely rule out the existence of a possible direct interaction between MKP1 and ATM. This interaction might facilitate the dephosphorylation and inactivation of ATM in unstressed conditions, thus preventing the untimely activation of checkpoint signaling. A co-immunoprecipitation assay in the presence and absence of $\mathrm{BCl}$ would give us more details into this aspect of MKP1 regulation of ATM.

Along with a possible direct interaction; we can also presume that ATM activation is a secondary effect of MKP1 inhibition. As mentioned previously one cannot set aside the participation of MK2 in regulating replication progression (see 6.1). Therefore, we can speculate that the replicative stress created by an activated MK2 might act as an additional trigger for ATM activation. Indeed, Olcina and co-workers have observed the activation of ATM in response to hypoxia-induced replicative stress. Since ATM is majorly known to be responsive against DNA damage arising from double stranded breaks, this piece of literature supports our results which also indicate a role for ATM in replicative stress (Olcina, Foskolou et al. 2013). Thus, if our theory with MK2 as an additional initiator for impaired replication holds true, triple inhibition with MKP1, MK2 and ATM would completely rescue the MKP1 inhibition-induced, down-regulation of fork speed. This can also be supplemented by an immunoblot experiment, where ATM phosphorylation (Ser 1981) can be monitored.

This makes way for our second question, how would ATM after activation, inhibit a progressing fork? During replicative stress, ATM acts to stabilize replication forks by facilitating chromatin remodeling and DNA repair (Costanzo, Robertson et al. 2000, Falck, Mailand et al. 2001, Groth, Lukas et al. 2003, Trenz, Smith et al. 2006, Olcina, Foskolou et al. 2013). However, our observations with ATM are pointing in the opposite direction, where we observe replicative stress as a consequence of ATM activation. How can we explain this phenomenon where ATM activation slows replication?

Since DNA helix unwinding is one of the crucial steps in fork elongation, it is convenient to assume that the MCM2-7 complex might be subjected to regulation by ATM. Infact, MCM2 and MCM3 are known to be phosphorylated by ATM at Ser 108 and Ser 535 respectively, after DNA damage (Cortez, Glick et al. 2004, Yoo, Shevchenko et al. 2004). Even though the functional consequence of such a phosphorylation is presently unclear; in the work conducted by Yoo et al., phosphorylation of Xenopus MCM at Ser 92 (Ser 108 in mammalians) inhibited DNA replication. Thus, we can presume the presence of such an inhibited MCM complex to mediate the effects seen due to ATM activation in our system. 
Possible additional link between ATM and replication could be certain proteins that negatively regulate fork progression. One such candidate is called Timeless alias Tim. Originally identified as a homologue to the drosophila TIMELESS gene product, involved in controlling circadian rhythm; Tim, along with its partner protein, Tipin (Tim interacting protein), was soon shown to be engaged in replication and cell cycle checkpoint response (Unsal-Kacmaz, Mullen et al. 2005, Chou and Elledge 2006, Yoshizawa-Sugata and Masai 2007, Smith, Fu et al. 2009). In 2007, Kacmaz and colleagues reported a 50\% reduction of DNA synthesis in undamaged, Tim depleted cells (Unsal-Kacmaz, Chastain et al. 2007). Invitro experiments using purified Tim showed an interaction of Tim with the DNA polymerases - pol $\alpha$, pol $\varepsilon$ and pol $\delta$, which stimulated the polymerase activity. This provided a potential explanation for the reduced fork speeds seen in Tim depleted cells (Aria, De Felice et al. 2013, Cho, Kang et al. 2013).

Despite lack of published reports on the existence of post translational modifications on Tim, protein sequence analysis using phosida (www.phosida.com) and phosphosite plus scan (www.phosphosite.org) reveal the presence of putative ATM/ATR phosphorylation motifs (i.e. 'SQ/TQ' motifs) on Tim - IPAKLS ${ }^{1087} \mathrm{PT}^{1089} \mathrm{QLRRAAA}$ (Highlighted grey portion shows the SQ/TQ motif). Therefore, it is conceivable that a negative regulation of this complex by ATM might also contribute to the replication impairment seen with MKP1 inhibition. To test if Tim-Tipin complex contributes to the effects seen in our system, a DNA fiber experiment using MKP1 inhibitor, $\mathrm{BCl}$, in cells depleted of Tim-Tipin can be carried out and analyzed for replication fork speeds. In such an experiment one would expect the $\mathrm{BCl}$ treated and the Tim-Tipin depleted samples to show reduced fork speeds. And, if our assumption holds true, the addition of $\mathrm{BCl}$ to a Tim/Tipin depleted sample would not have any further downregulatory effect on replication fork speed.

Moreover, the absence of Tim-Tipin complex has other functional consequences besides affecting DNA replication. This includes a loss of Chk1 phosphorylation by ATR in Tim or Tipin depleted cells, challenged with DNA damaging agents (Errico, Costanzo et al. 2007, Kemp, Akan et al. 2010). This piece of information fits well with our observations where a loss in Chk1 phosphorylation with MKP1 inhibitor and Gemcitabine, was not accompanied by a reduction in ATR activity or Claspin. This suggests that Chk1 regulation may be a consequence of a loss in Tim-Tipin activity (Appendix, Fig. 7.2, lanes 2 and 4; 6 and 8). Such a down-regulation of Chk1 activity can also result in inhibited fork progression (Ge and Blow 2010, Petermann, Woodcock et al. 2010).

Additionally, one could also imagine other components of the replisome associated with the progressing fork to be potential ATM substrates. Indeed, a large scale proteomic analysis done by the group of Stephan Elledge, reported an overwhelming - 700 phosphorylated 
substrates of ATM and ATR in response to DNA damage. Of these, 46 proteins were involved in DNA replication, recombination and repair (Matsuoka, Ballif et al. 2007). Later, Gamper et al., in 2010 identified an interaction between ATM and PCNA which positively regulated DNA polymerase $\delta$ and stimulated DNA synthesis (Gamper, Choi et al. 2012). Even though our data is incompatible with this particular finding, the presence of other putative ATM substrates which could be negatively regulated can still be considered.

Thus, in reference to our results we can speculate that a loss in MKP1 activity directly and indirectly (via MK2) stimulates ATM activity, which in turn inhibits DNA synthesis. This is possibly mediated by the various mechanisms mentioned above - phosphorylation of MCMs, loss of Tim/Tipin complex etc. However, the participation of these mechanisms in mediating our effects still needs to be verified. To further confirm our findings about ATM in DNA replication, we could also use a Wip1 inhibitor, to activate this kinase. Such an experiment will help us to learn if this negative role of ATM is only specific for MKP1 inhibition or if it is more universal.

Another interesting dimension to pursue will be the fate of these slowly replicating forks. Intuitively, one can imagine that these forks might stall, collapse or restart in the longer run. To monitor which is a likelier event to happen, we can quantify the percentage of stalled replication forks from a DNA fiber experiment, where cells are treated with MKP1 inhibitor for a long duration of time (see 4.4). Alternatively, since stalling of replication forks leaves behind long stretches of SSDNA, we can conduct a non-denaturizing BrdU uptake experiment, followed by immunofluorescence to calculate the percentage of ssDNA. Here, antibodies will be able to detect BrdU only in the open regions which have been created by the excessively unwound DNA near stalled forks.

A variation of the DNA fiber assay can be performed to check collapse/restart of stalled forks - here, after pre-incubation of the cells with MKP1 inhibitor; the first label will be applied. The cells will be left to recover in an inhibitor-free medium for a specific period of time, after which the second label will be applied. If replication forks collapse after treatment, second label will not be incorporated, thus giving us a measure of the collapsed forks (Petermann, Orta et al. 2010). In our studies, since long periods of MKP1 inhibitor treatment results in cell death, the probability of stalled forks collapsing seems higher.

\subsection{The disappearance of Cdc7}

MKP1 inhibition did not only affect progressing forks, but also inhibited replication initiation. As described in 6.1, while it remains to be seen if the reduction in origin firing upon MKP1 inhibition is dependent on MK2, we found out an additional replication initiation regulator, Cdc7 kinase, to be down-regulated after MKP1 inhibition. While this result looked promising 
initially, subsequent experiments done to verify this apparent down-regulation were inconclusive. This disappearance of $\mathrm{Cdc} 7$ seemed to be independent of proteasomal degradation or a loss in protein expression. To rule out the possibility of an unspecific binding by the antibody, a different clone was used that also yielded identical results (Appendix, Fig. 7.3). However, in reference to our mRNA expression studies, it should be noted that our data had high standard deviations, indicating the prevalence of some technical issues. Hence, the possibility of a down-regulation in Cdc7 expression upon MKP1 inhibition should not be completely negated. These results need to be verified by using different primer pairs as well. Moreover, the phosphorylation of MCM2 at Ser 53, which is mediated by Cdc7, is lost upon MKP1 inhibition, suggesting the involvement of Cdc7 in our system.

Co-incidentally, while performing MKP1 inhibition experiments in p53 deficient (HCT p53 -/-), p53 mutant (MiaPaCa-2) and p53 wt (HCT 116 p53 +/+) cell lines, we observed that Cdc7 and MCM2pS53 status, remains unchanged in both p53 null and mutant cell lines. On the other hand, p53 wt HCT cells showed a similar phenotype to that observed with U2OS cells, though not so profound. Strikingly, p53 was up-regulated upon MKP1 inhibition in the HCT116 p53 +/+ cells, suggesting the involvement of p53 in Cdc7 down-regulation (Appendix, Fig. 7.4). Supporting this belief is the inverse co-relation observed between p53 status in tumors and Cdc7 expression (Bonte, Lindvall et al. 2008, Wang and Simon 2013). Thus, this axis of $\mathrm{Cdc} 7$ regulation can be investigated further. One way to delineate this pathway would be to use MKP1 inhibition in U2OS cells transiently depleted of p53, and then look for Cdc7 levels.

Alternatively, Cdc7 might be phosphorylated upon MKP1 inhibition that masks the epitope of antibody binding. This might be a reason why the band intensity in these treated samples, appears weaker. Indeed, Cdc7 has four putative Cdk phosphorylation sites of which one residue, Thr 376, was found to be phosphorylated by Cdk/cyclinE in an in-vitro coimmunoprecipitation assay (Masai, Matsui et al. 2000). Even though this observation is in disagreement with our results as we would expect a decreased Cdk activity upon MKP1 inhibition, this does not rule out the possibility of a phosphorylation event existing.

Besides regulating origin firing, $\mathrm{Cdc} 7$ has also been reported to interact with and phosphorylate the p150 subunit of CAF1 (chromatin assembly factor-1). CAF1 is a histone chaperone which binds histones and assists nucleosome formation during DNA synthesis (Smith and Stillman 1989, Kaufman, Kobayashi et al. 1995, Verreault, Kaufman et al. 1996). The phosphorylation by Cdc7 promotes the association of CAF1 with PCNA, thereby targeting it to sites of DNA replication (Gérard, Koundrioukoff et al. 2006). Hence, we can presume that upon MKP1 inhibition, the loss of Cdc7, might also affect CAF1 and chromatin assembly. 
Additionally, Cdc7 has also been implicated in regulating the replication checkpoint, mainly involving the ATR-Chk1 pathway. Previous studies have shown its requirement in the activation of Chk1 in hydroxyurea (DNA synthesis inhibitor) treated cells (Kim, Kakusho et al. 2008). Recent work by Rainey et al., suggests that Cdc7 activity is also essential for maintaining interactions between Claspin and certain members of the replisome including MCMs (Rainey, Harhen et al. 2013). What is more attractive is the positive regulation of translesion synthesis by Cdc7. The Cdc7/Dbf4 complex phosphorylates Rad 18, which promotes its interaction with Pol $\eta$ and recruits it to the sites of stalled replication forks (Day, Palle et al. 2010, Vaziri and Masai 2010). Thus, a down-regulation of Cdc7 upon MKP1 inhibition, can affect not only DNA replication, but also checkpoint response and repair; making it an interesting area to explore further.

\subsection{Keep them close for survival: MKP1 and Mcl-1}

In addition to protecting cells from spontaneously arising replicative stress, our data suggests that MKP1 promotes cellular survival by safe-guarding an anti-apoptotic member of the Bcl2 family, Mcl-1, from proteasomal degradation. By doing so, a balance between the pool of pro-apoptotic and anti-apoptotic proteins is maintained, and cellular survival persists. What remains to be understood is how MKP1 being a phosphatase regulates Mcl-1.

As a first step towards learning this, we looked for a possible interaction between these proteins, which was clearly evident in the in-vitro co-immunoprecipitations using overexpression plasmids (see 5.12). This data needs to be confirmed in the native system as well. Besides this, localization studies will give us better insights into the dynamics of MKP1Mcl-1 interaction, as both of these proteins are found in cytosol, nucleus and mitochondria (Yang, Kozopas et al. 1995, Akgul, Moulding et al. 2000, Fujise, Zhang et al. 2000, Leuenroth, Grutkoski et al. 2000, Jamil, Sobouti et al. 2005, Wu, Zhang et al. 2005, Chattopadhyay, Machado-Pinilla et al. 2006, Thomas, Lam et al. 2010, Candas, Lu et al. 2014).

Given that they interact with each other, how does MKP1 protect Mcl-1 from degradation? What could be the molecular mechanism underlying the same? Mcl-1 is an exceptional member of the $\mathrm{Bcl} 2$ family because of its non-Bcl2-homologous, extended $\mathrm{N}$-terminus that is highly susceptible to various kinds of regulation due to the presence of 4 PEST regions (Proline/Glutamic acid/Serine/Threonine). In particular, phosphorylations on the Ser/Thr residues of these regions, differentially regulates the stability of $\mathrm{Mcl}-1$ (Michels, Johnson et al. 2005, Thomas, Lam et al. 2010). While some phosphorylations are known to stabilize this protein, a double phosphorylation at Ser 159 and Thr 163 in response to UV, makes it labile. In this case, phosphorylation at Thr 163 is mediated by JNKs while Ser 159 is mediated by 
GSK-3 $\beta$ - Glycogen Synthase Kinase-3 $\beta$ (Maurer, Charvet et al. 2006, Morel, Carlson et al. 2009). Similarly, a triple phosphorylation of Ser 155/Ser 159/Thr 163 mediated by GSK-3 $\beta$, also renders Mcl-1 unstable (Ding, He et al. 2007). Extrapolating this to our system, we can presume that a direct interaction of Mcl-1 with MKP1 facilitates the dephosphorylation of Mcl1 at these residues and keeps it stable. This argument is supported by the recent work of Nifousi and colleagues where Mcl-1 was shown to be de-phosphorylated at Thr 163/Ser 159 by an interaction with a different phosphatase, PP2A (Nifoussi, Ratcliffe et al. 2014). We can check this hypothesis in our setup, by analyzing the phosphorylated forms of Mcl-1 in a coimmunoprecipitation assay using MKP1 depleted cells, treated with proteasome inhibitor (to inhibit Mcl-1 degradation).

Instead of a direct interaction, another possibility could be the existence of MKP1 in a complex with both JNK and Mcl-1. In this way, MKP1 inactivates JNK and subsequently inhibits the priming phosphorylation required for Mcl-1 degradation. Since JNK is a primary substrate of MKP1, this idea also seems conceivable. Infact, this is consistent with our findings, where JNK is phosphorylated in the absence of MKP1 (Appendix, Fig. 7.5). Whether this affects the phosphorylation and subsequent degradation of Mcl-1, is still to be determined. One approach would be to deplete cells of both JNK and MKP1, and check if this would rescue MKP1 knock-down induced Mcl-1 degradation.

\subsection{Chemosensitization with MKP1 inhibition}

Various cancers including pancreatic, ovarian, lung and breast have an over-expression of MKP1 (see 2.8) which make them resistant to a wide range of chemotherapeutic drugs. In such a scenario, down-regulation of this protein would prove beneficial during chemotherapy. Since many of these drugs target DNA replication, combining them with MKP1 inhibition which produced similar effects, seemed like an attractive idea for achieving chemosensitization. Exploiting this, we used $\mathrm{MKP1}$ inhibitor, $\mathrm{BCl}$, in combination with a chemotherapeutic, gemcitabine, and monitored the effects on replication fork kinetics. Indeed, in our experiments, the combination of drugs slowed down replication rates to a larger extent than the single treatments. Such a massive reduction in DNA synthesis can activate the S-phase checkpoint leading to a cell-cycle arrest until the damage is resolved. In case of an irreparable, overwhelming damage, cells initiate apoptosis by activating the ATMChk2-p53 pathway (Karnitz, Flatten et al. 2005, Ewald, Sampath et al. 2008). Indeed, a long term proliferation assay (over a period of one week) using bright-field microscopy to quantify the cell confluence, can help analyze the rate of cellular survival after such a combined treatment. Since cancers mostly have deregulated checkpoint pathways, many-a-times, cells manage to escape the S-phase checkpoint and proceed prematurely to mitosis with an incompletely replicated genome. Under conditions where the mitotic checkpoint is also 
defective, cells go into mitotic catastrophe. Even a prolonged arrest at mitosis can lead to this phenomenon (Castedo, Perfettini et al. 2004, Mansilla, Bataller et al. 2006, Vitale, Galluzzi et al. 2011). While our preliminary results with the combination of MKP1 inhibitor and gemcitabine need to be consolidated further; this kind of combination treatment might indeed be advantageous for patients having an over-expression of MKP1.

Along this line, small molecules targeting MKP1 are being developed and tested in model systems. However, due to the structural similarity among the MKP family members, it is difficult to attain specificity towards any one protein. Often, most of the drugs target other closely related family members as well. Nonetheless, inhibiting more than one MKP might be favorable for the outcome of certain cancers which have a high expression of different MKPs. Some examples for MKP1 inhibitors include sanguinarine, NSC9537 and TPI-3, which show promising effects in in-vitro experiments. Of these, NSC9537 and TPI-3 seem to produce the best cytotoxic effects in cell culture systems (Nunes-Xavier, Roma-Mateo et al. 2011). To overcome the specificity problem, producing allosteric inhibitors (e.g. $\mathrm{BCl}$ for MKP1 and MKP3) which target a site other than the catalytic domain, seems like a worthy alternative for certain MKPs which rely on conformational changes for activation. Drugs targeting the kinase interaction motif at $\mathrm{N}$ - terminus which is required for substrate binding may also make the inhibition more specific. Apart from this, some agents like triptolide, a diterpenoid triepoxide, decrease the expression of MKP1 and have been shown to inhibit the proliferation of immortalized hippocampal cells (Koo, Kang et al. 2009). Triptolide was later shown to target a wide range of molecules including hsp70, Bax, $\mathrm{Bcl}-2, \mathrm{Mcl}-1$, and RNA polymerases which contributed to its effectiveness as an anti-cancer agent (R. 2014). While this was extremely encouraging, its poor solubility in water constrained its use in clinics. As an alternative, derivative of Triptolide, called Minnelide, has been reported to prevent tumor growth in mouse models of pancreatic cancer and osteosarcoma (Chugh, Sangwan et al. 2012, Banerjee, Thayanithy et al. 2013). Even though the involvement of MKP1 in this was undefined, considering its similarity to triptolide, one can imagine that it affects MKP1 expression as well. While MKP1 inhibitors may have a positive role in the treatment of cancers, owing to the dual function of MKP1 as a tumor suppressor or an oncogene (see 2.8), a major challenge still lies ahead before inhibitors are put forward for clinical trials.

\subsection{Conclusions: MKP1 in DNA replication, cellular survival and cancer}

Based on the results of this work, the function of MKP1 in DNA replication and survival can be summarized as follows: In an unperturbed cell cycle, MKP1 is required for inactivating the p38MAPK-MK2 and ATM-Chk2 pathway to maintain an optimal density of active forks progressing at normal speeds. This would be necessary to avoid the depletion of replication factors or nucleotides that might arise if too many origins fire or if a fork progresses too fast 
(Mantiero, Mackenzie et al. 2011, Poli, Tsaponina et al. 2012, Zhong, Nellimoottil et al. 2013). Additionally, MKP1 maintains stable levels of Cdc7, possibly to ensure complete genome replication by activating a sufficient number of origins. Thus, MKP1 is essential to shield the genome from spontaneously arising replicative stress. In an alternate pathway, MKP1 protects $\mathrm{Mcl}-1$ from degradation, and promotes cellular survival.

In the absence of MKP1, active ATM and MK2 promote replicative stress by altering fork dynamics and origin firing. This arrest in replication may lead to an accumulation of stalled forks that collapse over time, giving rise to irreparable DNA damage (Zeman and Cimprich 2014). Furthermore, due to the loss of additional proteins, Cdc7 and Mcl-1, MKP1-depleted cells suffer overwhelming stress and succumb to cell death. If these damaged cells manage to enter mitosis with an incompletely replicated genome, mitotic catastrophe (i.e. cell death due to mitotic failure) can ensue. This safe-guarding nature of MKP1 makes it an attractive druggable target to be used in combination with chemotherapeutics, for the treatment of cancer. 


\section{Appendix}

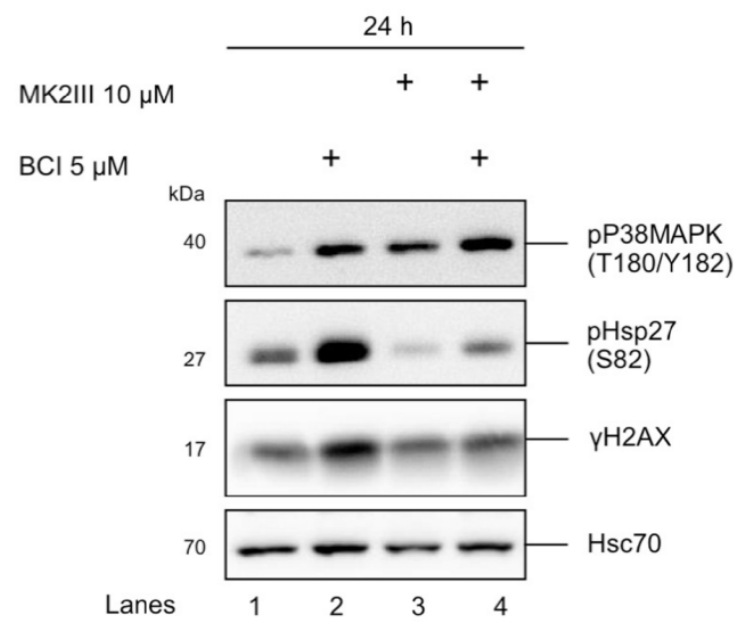

Figure 7.1 MK2 inhibition rescues the accumulation of phospho-H2AX (S139) upon MKP1 inhibition.

U2OS cells were exposed to $5 \mu \mathrm{M}$ in the presence or absence of MK2 inhibitor (MK2III) for $24 \mathrm{~h}$, and harvested for an immunoblot analysis against the above specified proteins. Hsc70 was used as a loading control.

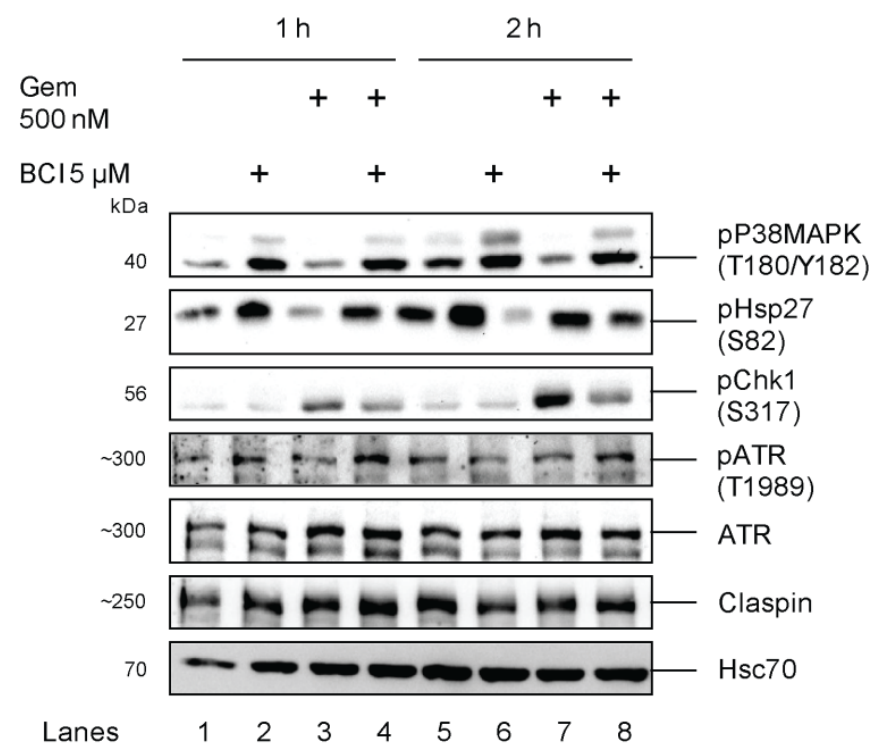

Figure 7.2 The loss of Chk1 phosphorylation upon MKP1 inhibition and Gemcitabine treatment is independent of ATR and Claspin.

U2OS cells were treated with $5 \mu \mathrm{M} \mathrm{BCl}, 500 \mathrm{nM}$ Gemcitabine or an equal volume of DMSO. Protein extracts were prepared after each of the indicated time points and subjected to an immunoblot analysis with the antibodies mentioned in the figure. Hsc70 served as a loading control. 


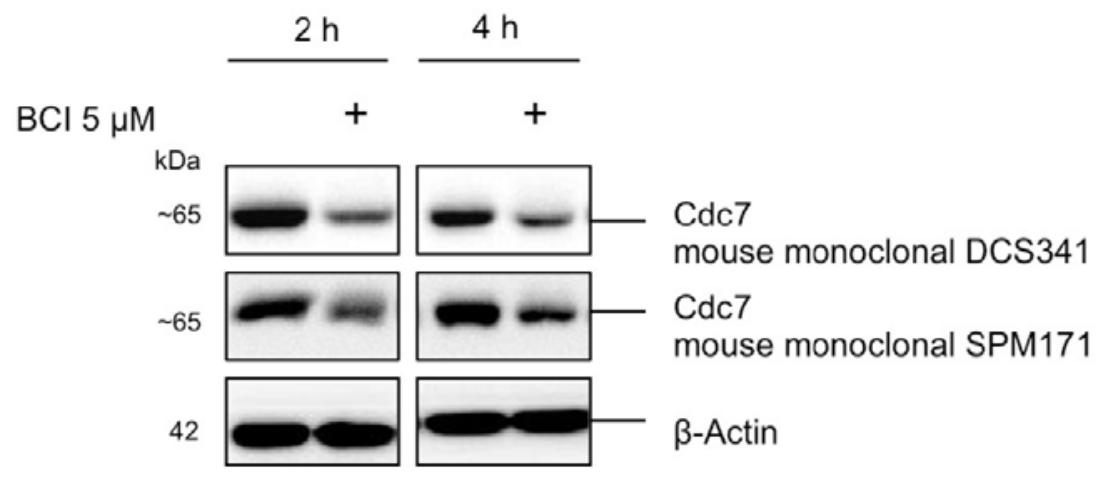

Figure 7.3 Different antibodies to Cdc7 yield similar patterns upon MKP1 inhibition.

U2OS cells were treated with $5 \mu \mathrm{M} \mathrm{BCl}$, or an equal volume of DMSO. Protein extracts prepared after each of the indicated time points were subjected to an immunoblot analysis with the different antibodies mentioned in the figure. Cdc7 DCS341 was from abcam (ab10535), while Cdc7 SPM171 was from Santa Cruz. $\beta$-Actin served as a loading control.

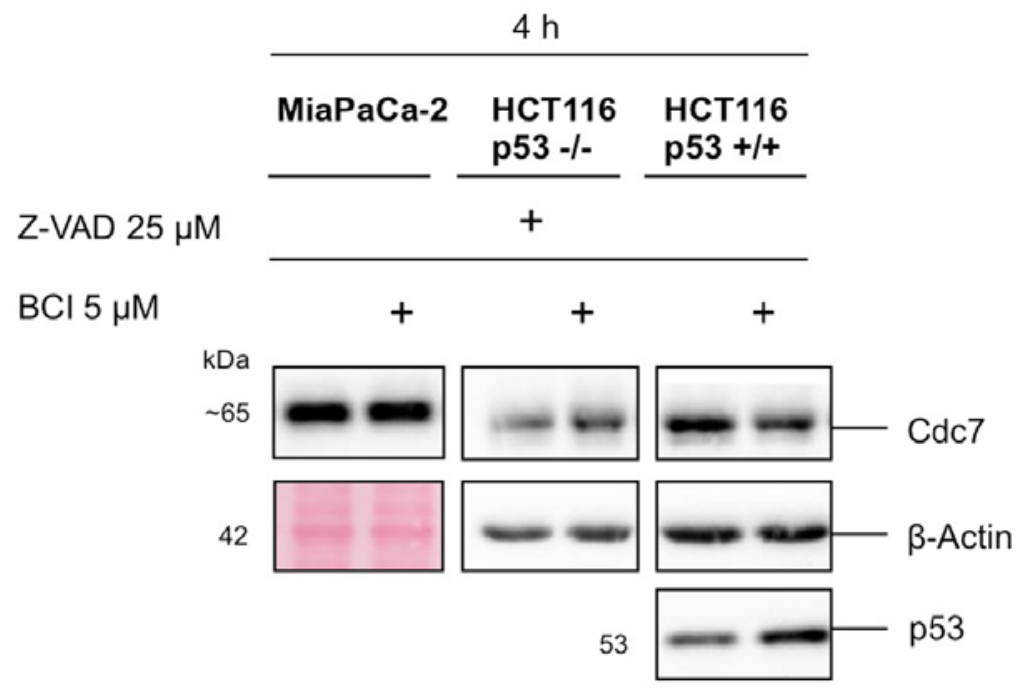

Figure 7.4 Activation of p53 may be responsible for the loss of Cdc7 observed with MKP1 inhibition.

MiaPaCa-2, HCT116 p53 -/- and HCT116 p53 +/+ cells were treated with $5 \mu \mathrm{M} \mathrm{BCl,} \mathrm{or} \mathrm{an} \mathrm{equal}$ volume of DMSO in the presence of a pan-caspase inhibitor Z-VAD-FMK for $4 \mathrm{~h}$. Cells were harvested for protein lysates after $4 \mathrm{~h}$ and an immunoblot analysis was done. Indicated antibodies were used. $\beta$ Actin served as a loading control. 


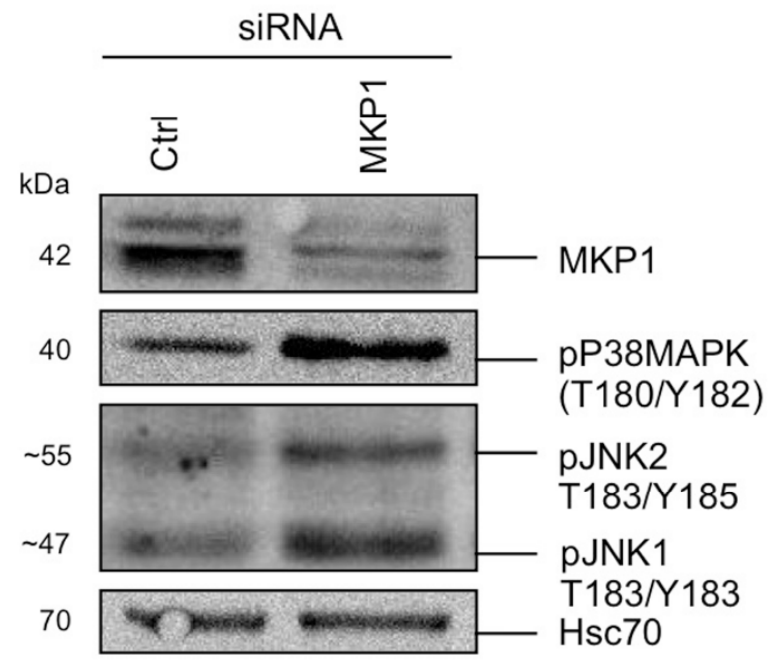

Figure 7.5 MKP1 knockdown leads to the activation of JNK.

U2OS cells were reverse transfected with a non-targeting siRNA or siRNA specific to MKP1 (designated as s4363, $10 \mathrm{nM}$ ) using lipofectamine and harvested after $48 \mathrm{~h}$ for immunoblot analysis. The membrane was probed with the indicated antibodies. Hsc70 was used as a loading control. 

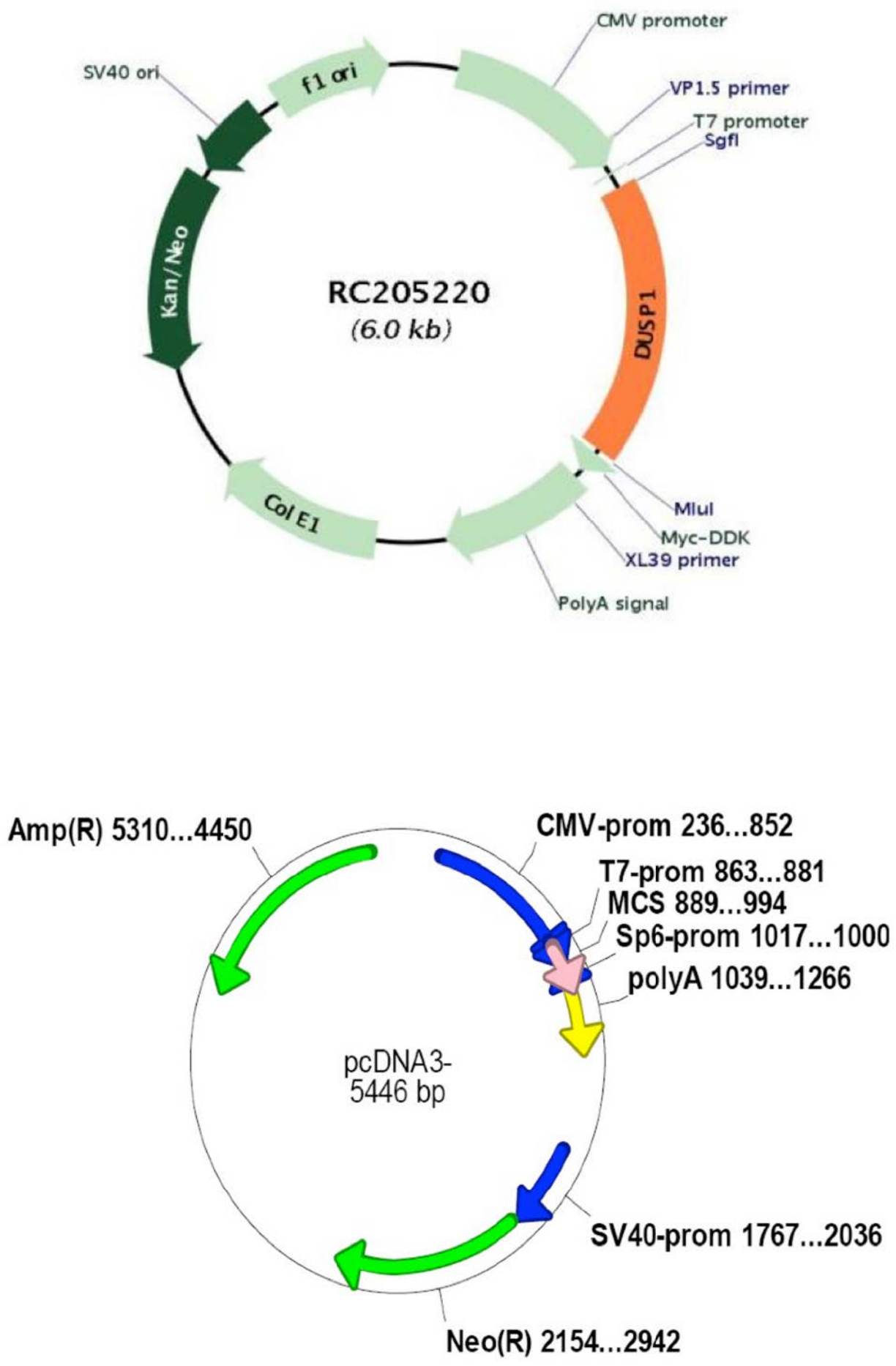

Figure 7.6 Plasmid maps of pCMV-DUSP1 and pcDNA3

The plasmid maps of PCMV-DUSP1 and PCDNA3 used in the over expression experiments have been shown. Additional information about the purchase of plasmids is given in materials (see 3.11). pcDNA3 was purchased from Invitrogen, Life technologies and pCMV-DUSP1 was purchased from Origene. 


\section{Abbreviations}

\begin{tabular}{|c|c|}
\hline${ }^{\circ} \mathrm{C}$ & Degree Celsius \\
\hline $9-1-1$ & Rad9-Hus1-Rad1 \\
\hline 53BP1 & P53 binding protein 1 \\
\hline$\mu g$ & Microgram \\
\hline$\mu \mathrm{L}$ & Microliter \\
\hline$\mu \mathrm{m}$ & Micrometer \\
\hline$\mu \mathrm{M}$ & Micromolar \\
\hline AP-1 & Activator protein 1 \\
\hline APAF1 & Apoptotic protease activating factor 1 \\
\hline APC & Anaphase promoting complex \\
\hline APS & Ammonium persulfate \\
\hline ATF2 & Activating transcription factor 2 \\
\hline ATM & Ataxia telangiectasia mutated \\
\hline ATP & Adenosine triphosphate \\
\hline ATR & ATM- and Rad3-related \\
\hline ATRIP & ATR interacting protein \\
\hline Bak & Bcl-2 homologous antagonist/killer \\
\hline Bax & Bcl-2-associated $X$ protein \\
\hline $\mathrm{BCA}$ & Bicinchoninic acid \\
\hline $\mathrm{Bcl}-2$ & B-cell lymphoma 2 \\
\hline Bcl-w & Bcl-2-like protein 2 \\
\hline $\mathrm{Bcl}-\mathrm{xL}$ & B-cell lymphoma-extra large \\
\hline $\mathrm{BH}$ & $\mathrm{Bcl} 2$ homology \\
\hline BLM & Bloom syndrome \\
\hline BRCA1 & Breast cancer susceptibility gene 1 \\
\hline BRCA2 & Breast cancer susceptibility gene 2 \\
\hline BrdU & Bromodeoxyuridine \\
\hline BSA & Bovine serum albumin \\
\hline CAD & Caspase activated DNase \\
\hline CAK & Cdk activating kinase \\
\hline CAF1 & Chromatin assembly factor 1 \\
\hline caspases & Cysteine aspartic proteases \\
\hline cDNA & Complementary DNA \\
\hline Cdk2 & Cyclin dependent kinase 2 \\
\hline Cdc6 & Cell division cycle 6 \\
\hline Cdc7 & Cell division cycle 7 \\
\hline Cdc25 & Cell division cycle 25 \\
\hline Cdc45 & Cell division cycle 45 \\
\hline Cdt1 & Chromatin licensing and DNA replication factor 1 \\
\hline Chk1 & Checkpoint kinase 1 \\
\hline Chk2 & Checkpoint kinase 2 \\
\hline Chk3 & Checkpoint kinase 3 \\
\hline CldU & Chlorodeoxyuridine \\
\hline $\mathrm{cm}$ & Centimeter \\
\hline
\end{tabular}




\begin{tabular}{|c|c|}
\hline ColP & Co-immunoprecipitation \\
\hline Crm1 & Chromosome region maintenance 1 \\
\hline ddNTP & Di-deoxy nucleoside triphosphate \\
\hline dNTP & Deoxy nucleoside triphosphate \\
\hline DDR & DNA damage response \\
\hline DHFR & Dihydrofolate reductase \\
\hline DMEM & Dulbecco's modified eagle's medium \\
\hline DMSO & Dimethyl sulfoxide \\
\hline DNA & Deoxyribonucleic acid \\
\hline DSB & Double strand break \\
\hline EDTA & Ethylene diamine tetraacetic acid \\
\hline EGFR & Epidermal growth factor receptor \\
\hline ERK & Extracellular-signal-regulated kinases \\
\hline $\mathrm{EtOH}$ & Ethanol \\
\hline EXO-11 & Exonuclease 11 \\
\hline FANCJ & Fanconi anemia group $\mathrm{J}$ \\
\hline Fas-L & Fas ligand \\
\hline FDA & Food and drug administration \\
\hline FCS & Fetal calf serum \\
\hline G1 & Gap1 \\
\hline G2 & Gap2 \\
\hline GINS & Go, Ichi, Nii, and San \\
\hline Glu & Glutamine \\
\hline 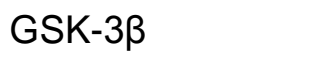 & Glycogen synthase kinase 3 \\
\hline $\mathrm{h}$ & Hour \\
\hline $\mathrm{H} 2 \mathrm{~A}$ & Histone 2A \\
\hline $\mathrm{H} 2 \mathrm{AX}$ & Histone 2AX \\
\hline HR & Homologous recombination \\
\hline $\mathrm{HCl}$ & Hydrochloric acid \\
\hline HRP & Horse radish peroxidase \\
\hline Hsp27 & Heat shock protein 27 \\
\hline IdU & lododeoxyuridine \\
\hline $\lg G$ & Immunoglobulin G \\
\hline Inh & Inhibitor \\
\hline JNK & C-Jun N-terminal kinase \\
\hline $\mathrm{kDa}$ & Kilodalton \\
\hline KIU & Kallikrein inactivator unit \\
\hline M & Molar \\
\hline M & Mitotic \\
\hline MAPK & Mitogen activated protein kinase \\
\hline MAPKK/MKK & MAPK kinase \\
\hline MAPKKK/ MKKK & MAPK kinase kinase \\
\hline Mcl-1 & Myeloid cell leukemia \\
\hline MCS & Multiple cloning site \\
\hline MCM & Minichromosome maintenance \\
\hline MDC-1 & Mediator of DNA damage checkpoint protein \\
\hline MEFs & Mouse embryonic fibroblasts \\
\hline MetOH & Methanol \\
\hline $\mathrm{mg}$ & Milligram \\
\hline
\end{tabular}




\begin{tabular}{|c|c|}
\hline $\min$ & Minute \\
\hline MK2 & MAPK activated protein kinase 2 \\
\hline MKP & MAPK phosphatase \\
\hline $\mathrm{mL}$ & Milliliter \\
\hline $\mathrm{mM}$ & Millimolar \\
\hline MOMP & Mitochondrial outer membrane permeabilization \\
\hline MRE11 & Meiotic recombination 11 \\
\hline MRN & Mre11 Rad50 Nbs1 \\
\hline mRNA & Messenger RNA \\
\hline MSK1 & Mitogen- and stress-activated protein kinase \\
\hline $\mathrm{n}$ & Sample size \\
\hline ng & Nanogram \\
\hline $\mathrm{nm}$ & Nanometer \\
\hline $\mathrm{nM}$ & Nanomolar \\
\hline NP-40 & Nonidet P-40 substitute \\
\hline ns & Non-significant \\
\hline NSCLC & Non-small cell lung carcinoma \\
\hline OMM & Outer mitochondrial membrane \\
\hline ORC & Origin recognition complex \\
\hline Ori & Origin \\
\hline $\mathrm{p}$ & Phospho \\
\hline PARP-1 & Poly (ADP-ribose) polymerase 1 \\
\hline PBS & Phosphate buffer saline \\
\hline PCNA & Proliferating cell nuclear antigen \\
\hline PCR & Polymerase chain reaction \\
\hline PGS & Protein G sepharose \\
\hline PIKK & Phosphatidylinositol 3-kinase related kinase \\
\hline Plk1 & Polo-like Kinase1 \\
\hline Pol & Polymerase \\
\hline PP1 & Protein phosphatase 1 \\
\hline PP2A & Protein phosphatase $2 \mathrm{~A}$ \\
\hline Pre-RC & Pre- replicative complex \\
\hline qRT-PCR & Quantitative reverse transcriptase PCR \\
\hline RFC & Replication factor C \\
\hline RNA & Ribonucleic acid \\
\hline RPA & Replication protein A \\
\hline rpm & Revolutions per min \\
\hline RT & Room temperature \\
\hline S & Synthesis \\
\hline SAPK & Stress activated protein kinase \\
\hline SCF & Skp, Cullin, F-box \\
\hline SDS & Sodium dodecyl sulfate \\
\hline SDS-PAGE & Sodium dodecyl sulfate polyacrylamide gel electrophoresis \\
\hline SiRNA & Small interfering RNA \\
\hline sSDNA & Single strand DNA \\
\hline TBS-T & Tris buffer saline with tween \\
\hline TEMED & Tetramethylethylenediamine \\
\hline Tim & Timeless \\
\hline Tipin & Timeless interacting protein \\
\hline
\end{tabular}




$\begin{array}{ll}\text { TLS } & \text { Translesion synthesis } \\ \text { TNF- } \alpha & \text { Tumor necrosis factor } \alpha \\ \text { TOPBP1 } & \text { Topoisomerase (DNA) II binding protein 1 } \\ \text { Tris } & \text { Trisamine } \\ \text { UV } & \text { Ultraviolet } \\ \text { Wip1 } & \text { Wild-type P53-induced phosphatase 1 } \\ \text { WRN } & \text { Werner } \\ \text { wt } & \text { Wildtype } \\ \text { YT } & \text { Yeast tryptone } \\ \text { YH2AX } & \text { H2AX phosphorylated on S319 }\end{array}$




\section{References}

Akgul, C., D. A. Moulding, M. R. White and S. W. Edwards (2000). "In vivo localisation and stability of human Mcl-1 using green fluorescent protein (GFP) fusion proteins." FEBS Lett 478(1-2): 72-76.

Alessi, D. R., C. Smythe and S. M. Keyse (1993). "The human CL100 gene encodes a Tyr/Thr-protein phosphatase which potently and specifically inactivates MAP kinase and suppresses its activation by oncogenic ras in Xenopus oocyte extracts." Oncogene 8(7): 2015-2020.

Aria, V., M. De Felice, R. Di Perna, S. Uno, H. Masai, J. E. Syvaoja, B. van Loon, U. Hubscher and F. M. Pisani (2013). "The human Tim-Tipin complex interacts directly with DNA polymerase epsilon and stimulates its synthetic activity." J Biol Chem 288(18): 1274212752.

Arias, E. E. and J. C. Walter (2005). "Replication-dependent destruction of Cdt1 limits DNA replication to a single round per cell cycle in Xenopus egg extracts." Genes \& Development 19(1): 114-126.

Bakkenist, C. J. and M. B. Kastan (2003). "DNA damage activates ATM through intermolecular autophosphorylation and dimer dissociation." Nature 421(6922): 499-506.

Banerjee, S., V. Thayanithy, V. Sangwan, T. N. Mackenzie, A. K. Saluja and S. Subramanian (2013). "Minnelide reduces tumor burden in preclinical models of osteosarcoma." Cancer Lett 335(2): 412-420.

Bartek, J., C. Lukas and J. Lukas (2004). "Checking on DNA damage in S phase." Nat Rev Mol Cell Biol 5(10): 792-804.

Bartek, J. and J. Lukas (2003). "Chk1 and Chk2 kinases in checkpoint control and cancer." Cancer Cell 3(5): 421-429.

Bermudez, O., G. Pages and C. Gimond (2010). "The dual-specificity MAP kinase phosphatases: critical roles in development and cancer." Am J Physiol Cell Physiol 299(2): C189-202.

Blow, J. J., X. Q. Ge and D. A. Jackson (2011). "How dormant origins promote complete genome replication." Trends Biochem Sci 36(8): 405-414.

Blow, J. J. and B. Hodgson (2002). "Replication licensing--defining the proliferative state?" Trends Cell Biol 12(2): 72-78.

Bonte, D., C. Lindvall, H. Liu, K. Dykema, K. Furge and M. Weinreich (2008). "Cdc7-Dbf4 kinase overexpression in multiple cancers and tumor cell lines is correlated with p53 inactivation." Neoplasia 10(9): 920-931.

Boutros, T., E. Chevet and P. Metrakos (2008). "Mitogen-activated protein (MAP) kinase/MAP kinase phosphatase regulation: roles in cell growth, death, and cancer." Pharmacol Rev 60(3): 261-310.

Brancho, D., N. Tanaka, A. Jaeschke, J. J. Ventura, N. Kelkar, Y. Tanaka, M. Kyuuma, T. Takeshita, R. A. Flavell and R. J. Davis (2003). "Mechanism of p38 MAP kinase activation in vivo." Genes Dev 17(16): 1969-1978.

Branzei, D. and M. Foiani (2009). "The checkpoint response to replication stress." DNA Repair 8(9): 1038-1046.

Bulavin, D. V., Y. Higashimoto, I. J. Popoff, W. A. Gaarde, V. Basrur, O. Potapova, E. Appella and A. J. Fornace, Jr. (2001). "Initiation of a G2/M checkpoint after ultraviolet radiation requires p38 kinase." Nature 411(6833): 102-107. 
Byun, T. S., M. Pacek, M. C. Yee, J. C. Walter and K. A. Cimprich (2005). "Functional uncoupling of MCM helicase and DNA polymerase activities activates the ATR-dependent checkpoint." Genes Dev 19(9): 1040-1052.

Candas, D., C. L. Lu, M. Fan, F. Y. Chuang, C. Sweeney, A. D. Borowsky and J. J. Li (2014). "Mitochondrial MKP1 is a target for therapy-resistant HER2-positive breast cancer cells." Cancer Res 74(24): 7498-7509.

Castedo, M., J. L. Perfettini, T. Roumier, K. Andreau, R. Medema and G. Kroemer (2004). "Cell death by mitotic catastrophe: a molecular definition." Oncogene 23(16): 2825-2837.

Chattopadhyay, S., R. Machado-Pinilla, C. Manguan-Garcia, C. Belda-Iniesta, C. Moratilla, P. Cejas, J. A. Fresno-Vara, J. de Castro-Carpeno, E. Casado, M. Nistal, M. Gonzalez-Baron and R. Perona (2006). "MKP1/CL100 controls tumor growth and sensitivity to cisplatin in non-small-cell lung cancer." Oncogene 25(23): 3335-3345.

Chinnaiyan, A. M. (1999). "The Apoptosome: Heart and Soul of the Cell Death Machine." Neoplasia (New York, N.Y.) 1(1): 5-15.

Chipuk, J. E., T. Moldoveanu, F. Llambi, M. J. Parsons and D. R. Green (2010). "The BCL-2 family reunion." Mol Cell 37(3): 299-310.

Cho, W. H., Y. H. Kang, Y. Y. An, I. Tappin, J. Hurwitz and J. K. Lee (2013). "Human TimTipin complex affects the biochemical properties of the replicative DNA helicase and DNA polymerases." Proc Natl Acad Sci U S A 110(7): 2523-2527.

Chou, D. M. and S. J. Elledge (2006). "Tipin and Timeless form a mutually protective complex required for genotoxic stress resistance and checkpoint function." Proc Natl Acad Sci U S A 103(48): 18143-18147.

Chugh, R., V. Sangwan, S. P. Patil, V. Dudeja, R. K. Dawra, S. Banerjee, R. J. Schumacher, B. R. Blazar, G. I. Georg, S. M. Vickers and A. K. Saluja (2012). "A preclinical evaluation of Minnelide as a therapeutic agent against pancreatic cancer." Sci Transl Med 4(156): 156 ra139.

Cook, J. G., D. A. Chasse and J. R. Nevins (2004). "The regulated association of Cdt1 with minichromosome maintenance proteins and Cdc6 in mammalian cells." J Biol Chem 279(10): 9625-9633.

Cortez, D., G. Glick and S. J. Elledge (2004). "Minichromosome maintenance proteins are direct targets of the ATM and ATR checkpoint kinases." Proc Natl Acad Sci U S A 101(27): 10078-10083.

Costanzo, V., K. Robertson, C. Y. Ying, E. Kim, E. Avvedimento, M. Gottesman, D. Grieco and J. Gautier (2000). "Reconstitution of an ATM-dependent checkpoint that inhibits chromosomal DNA replication following DNA damage." Mol Cell 6(3): 649-659.

Costanzo, V., D. Shechter, P. J. Lupardus, K. A. Cimprich, M. Gottesman and J. Gautier (2003). "An ATR- and Cdc7-dependent DNA damage checkpoint that inhibits initiation of DNA replication." Mol Cell 11(1): 203-213.

Coverley, D., C. Pelizon, S. Trewick and R. A. Laskey (2000). "Chromatin-bound Cdc6 persists in S and G2 phases in human cells, while soluble Cdc6 is destroyed in a cyclin Acdk2 dependent process." J Cell Sci 113 ( Pt 11): 1929-1938.

Dave, A., C. Cooley, M. Garg and A. Bianchi (2014). "Protein phosphatase 1 recruitment by Rif1 regulates DNA replication origin firing by counteracting DDK activity." Cell Rep 7(1): 5361.

Davis, R. J. (2000). "Signal transduction by the JNK group of MAP kinases." Cell 103(2): 239-252. 
Day, T. A., K. Palle, L. R. Barkley, N. Kakusho, Y. Zou, S. Tateishi, A. Verreault, H. Masai and C. Vaziri (2010). "Phosphorylated Rad18 directs DNA polymerase eta to sites of stalled replication." J Cell Biol 191(5): 953-966.

De Bont, R. and N. van Larebeke (2004). "Endogenous DNA damage in humans: a review of quantitative data." Mutagenesis 19(3): 169-185.

Derouet, M., L. Thomas, A. Cross, R. J. Moots and S. W. Edwards (2004). "Granulocyte macrophage colony-stimulating factor signaling and proteasome inhibition delay neutrophil apoptosis by increasing the stability of Mcl-1." J Biol Chem 279(26): 26915-26921.

Dhillon, A. S., S. Hagan, O. Rath and W. Kolch (2007). "MAP kinase signalling pathways in cancer." Oncogene 26(22): 3279-3290.

Ding, Q., X. He, J.-M. Hsu, W. Xia, C.-T. Chen, L.-Y. Li, D.-F. Lee, J.-C. Liu, Q. Zhong, X. Wang and M.-C. Hung (2007). "Degradation of Mcl-1 by $\beta$-TrCP Mediates Glycogen Synthase Kinase 3-Induced Tumor Suppression and Chemosensitization." Molecular and Cellular Biology 27(11): 4006-4017.

Elmore, S. (2007). "Apoptosis: A Review of Programmed Cell Death." Toxicologic pathology 35(4): 495-516.

Errico, A., V. Costanzo and T. Hunt (2007). "Tipin is required for stalled replication forks to resume DNA replication after removal of aphidicolin in Xenopus egg extracts." Proc Natl Acad Sci U S A 104(38): 14929-14934.

Espinosa, E., P. Zamora, J. Feliu and M. Gonzalez Baron (2003). "Classification of anticancer drugs--a new system based on therapeutic targets." Cancer Treat Rev 29(6): 515523.

Ewald, B., D. Sampath and W. Plunkett (2007). "H2AX phosphorylation marks gemcitabineinduced stalled replication forks and their collapse upon S-phase checkpoint abrogation." Cancer Ther 6(4): 1239-1248.

Ewald, B., D. Sampath and W. Plunkett (2008). "Nucleoside analogs: molecular mechanisms signaling cell death." Oncogene 27(50): 6522-6537.

Falck, J., N. Mailand, R. G. Syljuasen, J. Bartek and J. Lukas (2001). "The ATM-Chk2Cdc25A checkpoint pathway guards against radioresistant DNA synthesis." Nature 410(6830): 842-847.

Franklin, C. C., S. Srikanth and A. S. Kraft (1998). "Conditional expression of mitogenactivated protein kinase phosphatase-1, MKP-1, is cytoprotective against UV-induced apoptosis." Proceedings of the National Academy of Sciences of the United States of America 95(6): 3014-3019.

Fujise, K., D. Zhang, J. Liu and E. T. Yeh (2000). "Regulation of apoptosis and cell cycle progression by MCL1. Differential role of proliferating cell nuclear antigen." $\mathrm{J}$ Biol Chem 275(50): 39458-39465.

Gaestel, M. (2006). "MAPKAP kinases - MKs - two's company, three's a crowd." Nat Rev Mol Cell Biol 7(2): 120-130.

Gamper, A. M., S. Choi, Y. Matsumoto, D. Banerjee, A. E. Tomkinson and C. J. Bakkenist (2012). "ATM protein physically and functionally interacts with proliferating cell nuclear antigen to regulate DNA synthesis." J Biol Chem 287(15): 12445-12454.

Ge, X. Q. and J. J. Blow (2010). "Chk1 inhibits replication factory activation but allows dormant origin firing in existing factories." J Cell Biol 191(7): 1285-1297.

Ge, X. Q., D. A. Jackson and J. J. Blow (2007). "Dormant origins licensed by excess Mcm2-7 are required for human cells to survive replicative stress." Genes Dev 21(24): 3331-3341.

Gérard, A., S. Koundrioukoff, V. Ramillon, J.-C. Sergère, N. Mailand, J.-P. Quivy and G. Almouzni (2006). "The replication kinase Cdc7-Dbf4 promotes the interaction of the p150 
subunit of chromatin assembly factor 1 with proliferating cell nuclear antigen." EMBO Reports 7(8): 817-823.

Gilbert, D. M. (2004). "In search of the holy replicator." Nat Rev Mol Cell Biol 5(10): 848-855.

Gillespie, P. J. and J. J. Blow (2010). "Clusters, factories and domains: The complex structure of S phase comes into focus." Cell Cycle 9(16): 3218-3226.

Gillespie, P. J., A. Li and J. J. Blow (2001). "Reconstitution of licensed replication origins on Xenopus sperm nuclei using purified proteins." BMC Biochem 2: 15.

Goodarzi, A. A., P. Douglas, G. B. Moorhead and S. P. Lees-Miller (2007). "Utilizing protein phosphatase inhibitors to define PP2A as a regulator of ataxia-telangiectasia mutated." Methods Mol Biol 365: 47-59.

Goodarzi, A. A., J. C. Jonnalagadda, P. Douglas, D. Young, R. Ye, G. B. Moorhead, S. P. Lees-Miller and K. K. Khanna (2004). "Autophosphorylation of ataxia-telangiectasia mutated is regulated by protein phosphatase 2A." Embo i 23(22): 4451-4461.

Green, D. R. and G. I. Evan (2002). "A matter of life and death." Cancer Cell 1(1): 19-30.

Groth, A., J. Lukas, E. A. Nigg, H. H. Sillje, C. Wernstedt, J. Bartek and K. Hansen (2003). "Human Tousled like kinases are targeted by an ATM- and Chk1-dependent DNA damage checkpoint." Embo i 22(7): 1676-1687.

Hanahan, D. and R. A. Weinberg (2000). "The hallmarks of cancer." Cell 100(1): 57-70.

Hanahan, D. and R. A. Weinberg (2011). "Hallmarks of cancer: the next generation." Cell 144(5): 646-674.

Herrant, M., A. Jacquel, S. Marchetti, N. Belhacene, P. Colosetti, F. Luciano and P. Auberger (2004). "Cleavage of Mcl-1 by caspases impaired its ability to counteract Bim-induced apoptosis." Oncogene 23(47): 7863-7873.

Herrlich, P., M. Karin and C. Weiss (2008). "Supreme EnLIGHTenment: Damage Recognition and Signaling in the Mammalian UV Response." Molecular cell 29(3): 279-290.

Hoege, C., B. Pfander, G. L. Moldovan, G. Pyrowolakis and S. Jentsch (2002). "RAD6dependent DNA repair is linked to modification of PCNA by ubiquitin and SUMO." Nature 419(6903): 135-141.

Hu, J., N. Dang, T. Song and K. Vanderkerken (2011). "Mcl-1 reduction due to caspasedependent cleavage during endoplasmic reticulum stress-induced apoptosis." J Biol Chem 286(44): le24; author reply le25.

Hyrien, O., K. Marheineke and A. Goldar (2003). "Paradoxes of eukaryotic DNA replication: MCM proteins and the random completion problem." Bioessays 25(2): 116-125.

Ibarra, A., E. Schwob and J. Mendez (2008). "Excess MCM proteins protect human cells from replicative stress by licensing backup origins of replication." Proc Natl Acad Sci U S A 105(26): 8956-8961.

lizuka, M. and B. Stillman (1999). "Histone acetyltransferase HBO1 interacts with the ORC1 subunit of the human initiator protein." J Biol Chem 274(33): 23027-23034.

Jackson, D. A. and A. Pombo (1998). "Replicon Clusters Are Stable Units of Chromosome Structure: Evidence That Nuclear Organization Contributes to the Efficient Activation and Propagation of S Phase in Human Cells." The Journal of Cell Biology 140(6): 1285-1295.

Jackson, D. A. and A. Pombo (1998). "Replicon clusters are stable units of chromosome structure: evidence that nuclear organization contributes to the efficient activation and propagation of S phase in human cells." J Cell Biol 140(6): 1285-1295.

Jamil, S., R. Sobouti, P. Hojabrpour, M. Raj, J. Kast and V. Duronio (2005). "A proteolytic fragment of $\mathrm{Mcl}-1$ exhibits nuclear localization and regulates cell growth by interaction with Cdk1." Biochem J 387(Pt 3): 659-667. 
Jobson, A. G., G. T. Lountos, P. L. Lorenzi, J. Llamas, J. Connelly, D. Cerna, J. E. Tropea, A. Onda, G. Zoppoli, S. Kondapaka, G. Zhang, N. J. Caplen, J. H. Cardellina, 2nd, S. S. Yoo, A. Monks, C. Self, D. S. Waugh, R. H. Shoemaker and Y. Pommier (2009). "Cellular inhibition of checkpoint kinase 2 (Chk2) and potentiation of camptothecins and radiation by the novel Chk2 inhibitor PV1019 [7-nitro-1H-indole-2-carboxylic acid \{4-[1(guanidinohydrazone)-ethyl]-phenyl\}-amide]." J Pharmacol Exp Ther 331(3): 816-826.

Johnson, G. L. and R. Lapadat (2002). "Mitogen-activated protein kinase pathways mediated by ERK, JNK, and p38 protein kinases." Science 298(5600): 1911-1912.

Jones, R. M. and E. Petermann (2012). "Replication fork dynamics and the DNA damage response." Biochem J 443(1): 13-26.

Karnitz, L. M., K. S. Flatten, J. M. Wagner, D. Loegering, J. S. Hackbarth, S. J. Arlander, B. T. Vroman, M. B. Thomas, Y. U. Baek, K. M. Hopkins, H. B. Lieberman, J. Chen, W. A. Cliby and S. H. Kaufmann (2005). "Gemcitabine-induced activation of checkpoint signaling pathways that affect tumor cell survival." Mol Pharmacol 68(6): 1636-1644.

Kastan, M. B. and J. Bartek (2004). "Cell-cycle checkpoints and cancer." Nature 432(7015): 316-323.

Kaufman, P. D., R. Kobayashi, N. Kessler and B. Stillman (1995). "The p150 and p60 subunits of chromatin assembly factor I: a molecular link between newly synthesized histones and DNA replication." Cell 81(7): 1105-1114.

Kelland, L. (2007). "The resurgence of platinum-based cancer chemotherapy." Nat Rev Cancer 7(8): 573-584.

Kemp, M. G., Z. Akan, S. Yilmaz, M. Grillo, S. L. Smith-Roe, T. H. Kang, M. Cordeiro-Stone, W. K. Kaufmann, R. T. Abraham, A. Sancar and K. Unsal-Kacmaz (2010). "Tipin-replication protein A interaction mediates Chk1 phosphorylation by ATR in response to genotoxic stress." J Biol Chem 285(22): 16562-16571.

Kerr, J. F., A. H. Wyllie and A. R. Currie (1972). "Apoptosis: a basic biological phenomenon with wide-ranging implications in tissue kinetics." Br J Cancer 26(4): 239-257.

Keyse, S. M. and E. A. Emslie (1992). "Oxidative stress and heat shock induce a human gene encoding a protein-tyrosine phosphatase." Nature 359(6396): 644-647.

Kim, J. M., N. Kakusho, M. Yamada, Y. Kanoh, N. Takemoto and H. Masai (2008). "Cdc7 kinase mediates Claspin phosphorylation in DNA replication checkpoint." Oncogene 27(24): 3475-3482.

Koltun, E. S., A. L. Tsuhako, D. S. Brown, N. Aay, A. Arcalas, V. Chan, H. Du, S. Engst, K. Ferguson, M. Franzini, A. Galan, C. R. Holst, P. Huang, B. Kane, M. H. Kim, J. Li, D. Markby, M. Mohan, K. Noson, A. Plonowski, S. J. Richards, S. Robertson, K. Shaw, G. Stott, T. J. Stout, J. Young, P. Yu, C. A. Zaharia, W. Zhang, P. Zhou, J. M. Nuss, W. Xu and P. C. Kearney (2012). "Discovery of XL413, a potent and selective CDC7 inhibitor." Bioorg Med Chem Lett 22(11): 3727-3731.

Koo, H. S., S. D. Kang, J. H. Lee, N. H. Kim, H. T. Chung and H. O. Pae (2009). "Triptolide Inhibits the Proliferation of Immortalized HT22 Hippocampal Cells Via Persistent Activation of Extracellular Signal-Regulated Kinase-1/2 by Down-Regulating Mitogen-Activated Protein Kinase Phosphatase-1 Expression." J Korean Neurosurg Soc 46(4): 389-396.

Köpper, F. (2013). "The kinase MK2 in DNA replication upon genotoxic stress and chemotherapy." PhD thesis.

Kopper, F., C. Bierwirth, M. Schon, M. Kunze, I. Elvers, D. Kranz, P. Saini, M. B. Menon, D. Walter, C. S. Sorensen, M. Gaestel, T. Helleday, M. P. Schon and M. Dobbelstein (2013). "Damage-induced DNA replication stalling relies on MAPK-activated protein kinase 2 activity." Proc Natl Acad Sci U S A 110(42): 16856-16861. 
Kozlov, S. V., M. E. Graham, B. Jakob, F. Tobias, A. W. Kijas, M. Tanuji, P. Chen, P. J. Robinson, G. Taucher-Scholz, K. Suzuki, S. So, D. Chen and M. F. Lavin (2011). "Autophosphorylation and ATM Activation: ADDITIONAL SITES ADD TO THE COMPLEXITY." The Journal of Biological Chemistry 286(11): 9107-9119.

Kubota, Y., Y. Takase, Y. Komori, Y. Hashimoto, T. Arata, Y. Kamimura, H. Araki and H. Takisawa (2003). "A novel ring-like complex of Xenopus proteins essential for the initiation of DNA replication." Genes Dev 17(9): 1141-1152.

Laemmli, U. K. (1970). "Cleavage of Structural Proteins during the Assembly of the Head of Bacteriophage T4." Nature 227(5259): 680-685.

Lee, C., B. Hong, J. M. Choi, Y. Kim, S. Watanabe, Y. Ishimi, T. Enomoto, S. Tada, Y. Kim and Y. Cho (2004). "Structural basis for inhibition of the replication licensing factor Cdt1 by geminin." Nature 430(7002): 913-917.

Lei, M. and B. K. Tye (2001). "Initiating DNA synthesis: from recruiting to activating the MCM complex." J Cell Sci 114(Pt 8): 1447-1454.

Leonard, A. C. and M. Mechali (2013). "DNA replication origins." Cold Spring Harb Perspect Biol 5(10): a010116.

Leuenroth, S. J., P. S. Grutkoski, A. Ayala and H. H. Simms (2000). "The loss of Mcl-1 expression in human polymorphonuclear leukocytes promotes apoptosis." ㅈ Leukoc Biol 68(1): 158-166.

Li, C. J. and M. L. DePamphilis (2002). "Mammalian Orc1 protein is selectively released from chromatin and ubiquitinated during the S-to-M transition in the cell division cycle." Mol Cell Biol 22(1): 105-116.

Lin, X.-H., J. Walter, K. Scheidtmann, K. Ohst, J. Newport and G. Walter (1998). "Protein phosphatase $2 \mathrm{~A}$ is required for the initiation of chromosomal DNA replication." Proceedings of the National Academy of Sciences of the United States of America 95(25): 14693-14698.

Liu, E., X. Li, F. Yan, Q. Zhao and X. Wu (2004). "Cyclin-dependent kinases phosphorylate human Cdt1 and induce its degradation." J Biol Chem 279(17): 17283-17288.

Liu, Y., M. Gorospe, C. Yang and N. J. Holbrook (1995). "Role of mitogen-activated protein kinase phosphatase during the cellular response to genotoxic stress. Inhibition of c-Jun Nterminal kinase activity and AP-1-dependent gene activation." J Biol Chem 270(15): 83778380.

Machida, Y. J., J. L. Hamlin and A. Dutta (2005). "Right place, right time, and only once: replication initiation in metazoans." Cell 123(1): 13-24.

Majka, J. and P. M. Burgers (2004). "The PCNA-RFC families of DNA clamps and clamp loaders." Prog Nucleic Acid Res Mol Biol 78: 227-260.

Malhotra, V. and M. C. Perry (2003). "Classical chemotherapy: mechanisms, toxicities and the therapeutic window." Cancer Biol Ther 2(4 Suppl 1): S2-4.

Manke, I. A., A. Nguyen, D. Lim, M. Q. Stewart, A. E. Elia and M. B. Yaffe (2005). "MAPKAP kinase-2 is a cell cycle checkpoint kinase that regulates the G2/M transition and $S$ phase progression in response to UV irradiation." Mol Cell 17(1): 37-48.

Mansilla, S., M. Bataller and J. Portugal (2006). "Mitotic catastrophe as a consequence of chemotherapy." Anticancer Agents Med Chem 6(6): 589-602.

Mantiero, D., A. Mackenzie, A. Donaldson and P. Zegerman (2011). "Limiting replication initiation factors execute the temporal programme of origin firing in budding yeast." The EMBO Journal 30(23): 4805-4814.

Marheineke, K. and O. Hyrien (2004). "Control of replication origin density and firing time in Xenopus egg extracts: role of a caffeine-sensitive, ATR-dependent checkpoint." $\mathrm{J}$ Biol Chem 279(27): 28071-28081. 
Masai, H., E. Matsui, Z. You, Y. Ishimi, K. Tamai and K. Arai (2000). "Human Cdc7-related kinase complex. In vitro phosphorylation of MCM by concerted actions of Cdks and Cdc7 and that of a criticial threonine residue of Cdc7 bY Cdks." J Biol Chem 275(37): 29042-29052.

Matsuoka, S., B. A. Ballif, A. Smogorzewska, E. R. McDonald, 3rd, K. E. Hurov, J. Luo, C. E. Bakalarski, Z. Zhao, N. Solimini, Y. Lerenthal, Y. Shiloh, S. P. Gygi and S. J. Elledge (2007). "ATM and ATR substrate analysis reveals extensive protein networks responsive to DNA damage." Science 316(5828): 1160-1166.

Matsuura, K., M. Wakasugi, K. Yamashita and T. Matsunaga (2008). "Cleavage-mediated activation of Chk1 during apoptosis." J Biol Chem 283(37): 25485-25491.

Maurer, U., C. Charvet, A. S. Wagman, E. Dejardin and D. R. Green (2006). "Glycogen synthase kinase-3 regulates mitochondrial outer membrane permeabilization and apoptosis by destabilization of MCL-1." Mol Cell 21(6): 749-760.

Mazouzi, A., G. Velimezi and J. I. Loizou (2014). "DNA replication stress: causes, resolution and disease." Exp Cell Res 329(1): 85-93.

McClean, S., C. Costelloe, W. A. Denny, M. Searcey and L. P. Wakelin (1999). "Sequence selectivity, cross-linking efficiency and cytotoxicity of DNA-targeted 4-anilinoquinoline aniline mustards." Anticancer Drug Des 14(3): 187-204.

McGarry, T. J. and M. W. Kirschner (1998). "Geminin, an inhibitor of DNA replication, is degraded during mitosis." Cell 93(6): 1043-1053.

Mclntosh, D. and J. J. Blow (2012). "Dormant origins, the licensing checkpoint and the response to replicative stresses." Cold Spring Harbor perspectives in biology 4(10): 10.1101/cshperspect.a012955 a012955.

Mendez, J. and B. Stillman (2000). "Chromatin association of human origin recognition complex, cdc6, and minichromosome maintenance proteins during the cell cycle: assembly of prereplication complexes in late mitosis." Mol Cell Biol 20(22): 8602-8612.

Mendez, J. and B. Stillman (2003). "Perpetuating the double helix: molecular machines at eukaryotic DNA replication origins." Bioessays 25(12): 1158-1167.

Mendez, J., X. H. Zou-Yang, S. Y. Kim, M. Hidaka, W. P. Tansey and B. Stillman (2002). "Human origin recognition complex large subunit is degraded by ubiquitin-mediated proteolysis after initiation of DNA replication." Mol Cell 9(3): 481-491.

Michels, J., P. W. Johnson and G. Packham (2005). "Mcl-1." Int J Biochem Cell Biol 37(2): 267-271.

Molina, G., A. Vogt, A. Bakan, W. Dai, P. Queiroz de Oliveira, W. Znosko, T. E. Smithgall, I. Bahar, J. S. Lazo, B. W. Day and M. Tsang (2009). "Zebrafish chemical screening reveals an inhibitor of Dusp6 that expands cardiac cell lineages." Nat Chem Biol 5(9): 680-687.

Montagnoli, A., B. Valsasina, D. Brotherton, S. Troiani, S. Rainoldi, P. Tenca, A. Molinari and C. Santocanale (2006). "Identification of Mcm2 phosphorylation sites by S-phase-regulating kinases." J Biol Chem 281(15): 10281-10290.

Montagnoli, A., B. Valsasina, V. Croci, M. Menichincheri, S. Rainoldi, V. Marchesi, M. Tibolla, P. Tenca, D. Brotherton, C. Albanese, V. Patton, R. Alzani, A. Ciavolella, F. Sola, A. Molinari, D. Volpi, N. Avanzi, F. Fiorentini, M. Cattoni, S. Healy, D. Ballinari, E. Pesenti, A. Isacchi, J. Moll, A. Bensimon, E. Vanotti and C. Santocanale (2008). "A Cdc7 kinase inhibitor restricts initiation of DNA replication and has antitumor activity." Nat Chem Biol 4(6): 357-365.

Moore, M. J., D. Goldstein, J. Hamm, A. Figer, J. R. Hecht, S. Gallinger, H. J. Au, P. Murawa, D. Walde, R. A. Wolff, D. Campos, R. Lim, K. Ding, G. Clark, T. VoskoglouNomikos, M. Ptasynski and W. Parulekar (2007). "Erlotinib plus gemcitabine compared with gemcitabine alone in patients with advanced pancreatic cancer: a phase III trial of the National Cancer Institute of Canada Clinical Trials Group." J Clin Oncol 25(15): 1960-1966. 
Morel, C., S. M. Carlson, F. M. White and R. J. Davis (2009). "Mcl-1 integrates the opposing actions of signaling pathways that mediate survival and apoptosis." Mol Cell Biol 29(14): 3845-3852.

Nicholson, D. W. (1999). "Caspase structure, proteolytic substrates, and function during apoptotic cell death." Cell Death Differ 6(11): 1028-1042.

Nifoussi, S. K., N. R. Ratcliffe, D. L. Ornstein, G. Kasof, S. Strack and R. W. Craig (2014). "Inhibition of protein phosphatase 2A (PP2A) prevents Mcl-1 protein dephosphorylation at the Thr-163/Ser-159 phosphodegron, dramatically reducing expression in Mcl-1-amplified lymphoma cells." J Biol Chem 289(32): 21950-21959.

Nijhawan, D., M. Fang, E. Traer, Q. Zhong, W. Gao, F. Du and X. Wang (2003). "Elimination of Mcl-1 is required for the initiation of apoptosis following ultraviolet irradiation." Genes Dev 17(12): 1475-1486.

Nimonkar, A. V., J. Genschel, E. Kinoshita, P. Polaczek, J. L. Campbell, C. Wyman, P. Modrich and S. C. Kowalczykowski (2011). "BLM-DNA2-RPA-MRN and EXO1-BLM-RPAMRN constitute two DNA end resection machineries for human DNA break repair." Genes Dev 25(4): 350-362.

Norbury, C. J. and B. Zhivotovsky (2004). "DNA damage-induced apoptosis." Oncogene 23(16): 2797-2808.

Nunes-Xavier, C., C. Roma-Mateo, P. Rios, C. Tarrega, R. Cejudo-Marin, L. Tabernero and R. Pulido (2011). "Dual-specificity MAP kinase phosphatases as targets of cancer treatment." Anticancer Agents Med Chem 11(1): 109-132.

Olcina, M. M., I. P. Foskolou, S. Anbalagan, J. M. Senra, I. M. Pires, Y. Jiang, A. J. Ryan and E. M. Hammond (2013). "Replication stress and chromatin context link ATM activation to a role in DNA replication." Mol Cell 52(5): 758-766.

Owens, D. M. and S. M. Keyse (2007). "Differential regulation of MAP kinase signalling by dual-specificity protein phosphatases." Oncogene 26(22): 3203-3213.

Parrilla-Castellar, E. R., S. J. Arlander and L. Karnitz (2004). "Dial 9-1-1 for DNA damage: the Rad9-Hus1-Rad1 (9-1-1) clamp complex." DNA Repair (Amst) 3(8-9): 1009-1014.

Petermann, E., M. L. Orta, N. Issaeva, N. Schultz and T. Helleday (2010). "Hydroxyureastalled replication forks become progressively inactivated and require two different RAD51mediated pathways for restart and repair." Mol Cell 37(4): 492-502.

Petermann, E., M. Woodcock and T. Helleday (2010). "Chk1 promotes replication fork progression by controlling replication initiation." Proc Natl Acad Sci U S A 107(37): 1609016095.

Petersen, B. O., J. Lukas, C. S. Sørensen, J. Bartek and K. Helin (1999). "Phosphorylation of mammalian CDC6 by cyclin A/CDK2 regulates its subcellular localization." The EMBO Journal 18(2): 396-410.

Petersen, P., D. M. Chou, Z. You, T. Hunter, J. C. Walter and G. Walter (2006). "Protein phosphatase 2A antagonizes ATM and ATR in a Cdk2- and Cdc7-independent DNA damage checkpoint." Mol Cell Biol 26(5): 1997-2011.

Poli, J., O. Tsaponina, L. Crabbe, A. Keszthelyi, V. Pantesco, A. Chabes, A. Lengronne and P. Pasero (2012). "dNTP pools determine fork progression and origin usage under replication stress." Embo i 31(4): 883-894.

Prakash, S., R. E. Johnson and L. Prakash (2005). "Eukaryotic translesion synthesis DNA polymerases: specificity of structure and function." Annu Rev Biochem 74: 317-353.

R., H. (2014). "Triptolide: Novel Anticancer Agent for Chemoresistant Cancer Cells that are Caspase-3

Deficient." J Mol Biol \& Mol Imaging. 1(3): 8. 
Rainey, M., B. Harhen, G.-N. Wang, P. Murphy and C. Santocanale (2013). "Cdc7dependent and -independent phosphorylation of Claspin in the induction of the DNA replication checkpoint." Cell Cycle 12(10): 1560-1568.

Reinhardt, H. C., A. S. Aslanian, J. A. Lees and M. B. Yaffe (2007). "p53-deficient cells rely on ATM- and ATR-mediated checkpoint signaling through the p38MAPK/MK2 pathway for survival after DNA damage." Cancer Cell 11(2): 175-189.

Reinhardt, H. C., P. Hasskamp, I. Schmedding, S. Morandell, M. A. van Vugt, X. Wang, R. Linding, S. E. Ong, D. Weaver, S. A. Carr and M. B. Yaffe (2010). "DNA damage activates a spatially distinct late cytoplasmic cell-cycle checkpoint network controlled by MK2-mediated RNA stabilization." Mol Cell 40(1): 34-49.

Reinhardt, H. C. and M. B. Yaffe (2009). "Kinases that control the cell cycle in response to DNA damage: Chk1, Chk2, and MK2." Curr Opin Cell Biol 21(2): 245-255.

Remus, D. and J. F. Diffley (2009). "Eukaryotic DNA replication control: lock and load, then fire." Curr Opin Cell Biol 21(6): 771-777.

Rich, T., R. L. Allen and A. H. Wyllie (2000). "Defying death after DNA damage." Nature 407(6805): 777-783.

Riedl, S. J. and G. S. Salvesen (2007). "The apoptosome: signalling platform of cell death." Nat Rev Mol Cell Biol 8(5): 405-413.

Roberts, P. J. and C. J. Der (2007). "Targeting the Raf-MEK-ERK mitogen-activated protein kinase cascade for the treatment of cancer." Oncogene 26(22): 3291-3310.

Rogakou, E. P., D. R. Pilch, A. H. Orr, V. S. Ivanova and W. M. Bonner (1998). "DNA doublestranded breaks induce histone H2AX phosphorylation on serine 139." J Biol Chem 273(10): 5858-5868.

Rowles, A., J. P. Chong, L. Brown, M. Howell, G. I. Evan and J. J. Blow (1996). "Interaction between the origin recognition complex and the replication licensing system in Xenopus." Cell 87(2): 287-296.

Saelens, X., N. Festjens, L. Vande Walle, M. van Gurp, G. van Loo and P. Vandenabeele (2004). "Toxic proteins released from mitochondria in cell death." Oncogene 23(16): 28612874.

Saha, P., J. Chen, K. C. Thome, S. J. Lawlis, Z. H. Hou, M. Hendricks, J. D. Parvin and A. Dutta (1998). "Human CDC6/Cdc18 associates with Orc1 and cyclin-cdk and is selectively eliminated from the nucleus at the onset of S phase." Mol Cell Biol 18(5): 2758-2767.

Saintigny, Y., F. Delacôte, G. Varès, F. Petitot, S. Lambert, D. Averbeck and B. S. Lopez (2001). "Characterization of homologous recombination induced by replication inhibition in mammalian cells." The EMBO Journal 20(14): 3861-3870.

Sakahira, H., M. Enari and S. Nagata (1998). "Cleavage of CAD inhibitor in CAD activation and DNA degradation during apoptosis." Nature 391(6662): 96-99.

Sanchez-Perez, I., M. Martinez-Gomariz, D. Williams, S. M. Keyse and R. Perona (2000). "CL100/MKP-1 modulates JNK activation and apoptosis in response to cisplatin." Oncogene 19(45): 5142-5152.

Sanger, F., S. Nicklen and A. R. Coulson (1977). "DNA sequencing with chain-terminating inhibitors." Proc Natl Acad Sci U S A 74(12): 5463-5467.

Schlacher, K., N. Christ, N. Siaud, A. Egashira, H. Wu and M. Jasin (2011). "Double-strand break repair-independent role for BRCA2 in blocking stalled replication fork degradation by MRE11." Cell 145(4): 529-542.

Schlacher, K., H. Wu and M. Jasin (2012). "A Distinct Replication Fork Protection Pathway Connects Fanconi Anemia Tumor Suppressors to RAD51-BRCA1/2." Cancer cell 22(1): 106116. 
Sharma, V. and J. J. Tepe (2004). "Potent inhibition of checkpoint kinase activity by a hymenialdisine-derived indoloazepine." Bioorg Med Chem Lett 14(16): 4319-4321.

Shechter, D., V. Costanzo and J. Gautier (2004). "ATR and ATM regulate the timing of DNA replication origin firing." Nat Cell Biol 6(7): 648-655.

Shreeram, S., O. N. Demidov, W. K. Hee, H. Yamaguchi, N. Onishi, C. Kek, O. N. Timofeev, C. Dudgeon, A. J. Fornace, C. W. Anderson, Y. Minami, E. Appella and D. V. Bulavin (2006). "Wip1 phosphatase modulates ATM-dependent signaling pathways." Mol Cell 23(5): 757764.

Sigurdsson, S., S. Van Komen, W. Bussen, D. Schild, J. S. Albala and P. Sung (2001). "Mediator function of the human Rad51B-Rad51C complex in Rad51/RPA-catalyzed DNA strand exchange." Genes Dev 15(24): 3308-3318.

Slack, D. N., O. M. Seternes, M. Gabrielsen and S. M. Keyse (2001). "Distinct binding determinants for ERK2/p38alpha and JNK map kinases mediate catalytic activation and substrate selectivity of map kinase phosphatase-1." J Biol Chem 276(19): 16491-16500.

Small, G. W., Y. Y. Shi, L. S. Higgins and R. Z. Orlowski (2007). "Mitogen-activated protein kinase phosphatase-1 is a mediator of breast cancer chemoresistance." Cancer Res 67(9): 4459-4466.

Smith, K. D., M. A. Fu and E. J. Brown (2009). "Tim-Tipin dysfunction creates an indispensible reliance on the ATR-Chk1 pathway for continued DNA synthesis." $\mathrm{J}$ Cell Biol 187(1): 15-23.

Smith, S. and B. Stillman (1989). "Purification and characterization of CAF-I, a human cell factor required for chromatin assembly during DNA replication in vitro." Cell 58(1): 15-25.

Song, B., X. S. Liu, K. Davis and X. Liu (2011). "Plk1 phosphorylation of Orc2 promotes DNA replication under conditions of stress." Mol Cell Biol 31(23): 4844-4856.

Sørensen, C. S. and R. G. Syljuåsen (2012). "Safeguarding genome integrity: the checkpoint kinases ATR, CHK1 and WEE1 restrain CDK activity during normal DNA replication." Nucleic Acids Research 40(2): 477-486.

Sorensen, C. S., R. G. Syljuasen, J. Lukas and J. Bartek (2004). "ATR, Claspin and the Rad9-Rad1-Hus1 complex regulate Chk1 and Cdc25A in the absence of DNA damage." Cell Cycle 3(7): 941-945.

Stiff, T., S. A. Walker, K. Cerosaletti, A. A. Goodarzi, E. Petermann, P. Concannon, M. O'Driscoll and P. A. Jeggo (2006). "ATR-dependent phosphorylation and activation of ATM in response to UV treatment or replication fork stalling." The EMBO Journal 25(24): 5775-5782.

Stokoe, D., K. Engel, D. G. Campbell, P. Cohen and M. Gaestel (1992). "Identification of MAPKAP kinase 2 as a major enzyme responsible for the phosphorylation of the small mammalian heat shock proteins." FEBS Lett 313(3): 307-313.

Syljuasen, R. G., C. S. Sorensen, L. T. Hansen, K. Fugger, C. Lundin, F. Johansson, T. Helleday, M. Sehested, J. Lukas and J. Bartek (2005). "Inhibition of human Chk1 causes increased initiation of DNA replication, phosphorylation of ATR targets, and DNA breakage." Mol Cell Biol 25(9): 3553-3562.

Takeda, D. Y., J. D. Parvin and A. Dutta (2005). "Degradation of Cdt1 during S phase is Skp2-independent and is required for efficient progression of mammalian cells through $S$ phase." J Biol Chem 280(24): 23416-23423.

Takeuchi, K., T. Shin-ya, K. Nishio and F. Ito (2009). "Mitogen-activated protein kinase phosphatase-1 modulated JNK activation is critical for apoptosis induced by inhibitor of epidermal growth factor receptor-tyrosine kinase." Febs i 276(5): 1255-1265.

Taylor, R. C., S. P. Cullen and S. J. Martin (2008). "Apoptosis: controlled demolition at the cellular level." Nat Rev Mol Cell Biol 9(3): 231-241. 
Thiriet, C. and J. J. Hayes (2005). "Chromatin in need of a fix: phosphorylation of H2AX connects chromatin to DNA repair." Mol Cell 18(6): 617-622.

Thomas, L. W., C. Lam and S. W. Edwards (2010). "Mcl-1; the molecular regulation of protein function." FEBS Lett 584(14): 2981-2989.

Towbin, H., T. Staehelin and J. Gordon (1979). "Electrophoretic transfer of proteins from polyacrylamide gels to nitrocellulose sheets: procedure and some applications." Proc Natl Acad Sci U S A 76(9): 4350-4354.

Trenz, K., A. Errico and V. Costanzo (2008). "PIx1 is required for chromosomal DNA replication under stressful conditions." The EMBO Journal 27(6): 876-885.

Trenz, K., E. Smith, S. Smith and V. Costanzo (2006). "ATM and ATR promote Mre11 dependent restart of collapsed replication forks and prevent accumulation of DNA breaks." Embo i 25(8): 1764-1774.

Tsuji, T., S. B. Ficarro and W. Jiang (2006). "Essential role of phosphorylation of MCM2 by Cdc7/Dbf4 in the initiation of DNA replication in mammalian cells." Mol Biol Cell 17(10): 4459-4472.

Tsuyama, T., S. Tada, S. Watanabe, M. Seki and T. Enomoto (2005). "Licensing for DNA replication requires a strict sequential assembly of Cdc6 and Cdt1 onto chromatin in Xenopus egg extracts." Nucleic Acids Res 33(2): 765-775.

Unsal-Kacmaz, K., P. D. Chastain, P. P. Qu, P. Minoo, M. Cordeiro-Stone, A. Sancar and W. K. Kaufmann (2007). "The human Tim/Tipin complex coordinates an Intra-S checkpoint response to UV that slows replication fork displacement." Mol Cell Biol 27(8): 3131-3142.

Unsal-Kacmaz, K., T. E. Mullen, W. K. Kaufmann and A. Sancar (2005). "Coupling of human circadian and cell cycles by the timeless protein." Mol Cell Biol 25(8): 3109-3116.

Vaziri, C. and H. Masai (2010). "Integrating DNA replication with trans-lesion synthesis via Cdc7." Cell Cycle 9(24): 4818-4823.

Verreault, A., P. D. Kaufman, R. Kobayashi and B. Stillman (1996). "Nucleosome assembly by a complex of CAF-1 and acetylated histones H3/H4." Cell 87(1): 95-104.

Vitale, I., L. Galluzzi, M. Castedo and G. Kroemer (2011). "Mitotic catastrophe: a mechanism for avoiding genomic instability." Nat Rev Mol Cell Biol 12(6): 385-392.

Wagner, E. F. and A. R. Nebreda (2009). "Signal integration by JNK and p38 MAPK pathways in cancer development." Nat Rev Cancer 9(8): 537-549.

Walter, J. and J. Newport (2000). "Initiation of eukaryotic DNA replication: origin unwinding and sequential chromatin association of Cdc45, RPA, and DNA polymerase alpha." Mol Cell 5(4): 617-627.

Wang, X. and R. Simon (2013). "Identification of potential synthetic lethal genes to p53 using a computational biology approach." BMC Med Genomics 6: 30.

Wang, Z., J. Y. Zhou, D. Kanakapalli, S. Buck, G. S. Wu and Y. Ravindranath (2008). "High level of mitogen-activated protein kinase phosphatase-1 expression is associated with cisplatin resistance in osteosarcoma." Pediatr Blood Cancer 51(6): 754-759.

Ward, I. M. and J. Chen (2001). "Histone H2AX is phosphorylated in an ATR-dependent manner in response to replicational stress." J Biol Chem 276(51): 47759-47762.

Watanabe, N., M. Broome and T. Hunter (1995). "Regulation of the human WEE1Hu CDK tyrosine 15-kinase during the cell cycle." Embo i 14(9): 1878-1891.

Weng, C., Y. Li, D. Xu, Y. Shi and H. Tang (2005). "Specific cleavage of Mcl-1 by caspase-3 in tumor necrosis factor-related apoptosis-inducing ligand (TRAIL)-induced apoptosis in Jurkat leukemia T cells." J Biol Chem 280(11): 10491-10500. 
Willis, S. N., L. Chen, G. Dewson, A. Wei, E. Naik, J. I. Fletcher, J. M. Adams and D. C. Huang (2005). "Proapoptotic Bak is sequestered by Mcl-1 and $\mathrm{Bcl}-\mathrm{xL}$, but not Bcl-2, until displaced by BH3-only proteins." Genes Dev 19(11): 1294-1305.

Wilson, T. R., D. B. Longley and P. G. Johnston (2006). "Chemoresistance in solid tumours." Ann Oncol 17 Suppl 10: x315-324.

Woodward, A. M., T. Gohler, M. G. Luciani, M. Oehlmann, X. Ge, A. Gartner, D. A. Jackson and J. J. Blow (2006). "Excess Mcm2-7 license dormant origins of replication that can be used under conditions of replicative stress." J Cell Biol 173(5): 673-683.

Wu, G. S. (2007). "Role of mitogen-activated protein kinase phosphatases (MKPS) in cancer." Cancer Metastasis Rev 26(3-4): 579-585.

Wu, J. J. and A. M. Bennett (2005). "Essential role for mitogen-activated protein (MAP) kinase phosphatase-1 in stress-responsive MAP kinase and cell survival signaling." $\mathrm{J}$ Biol Chem 280(16): 16461-16466.

Wu, J. J., L. Zhang and A. M. Bennett (2005). "The noncatalytic amino terminus of mitogenactivated protein kinase phosphatase 1 directs nuclear targeting and serum response element transcriptional regulation." Mol Cell Biol 25(11): 4792-4803.

Yanagi, K., T. Mizuno, Z. You and F. Hanaoka (2002). "Mouse geminin inhibits not only Cdt1MCM6 interactions but also a novel intrinsic Cdt1 DNA binding activity." J Biol Chem 277(43): 40871-40880.

Yang, T., K. M. Kozopas and R. W. Craig (1995). "The intracellular distribution and pattern of expression of Mcl-1 overlap with, but are not identical to, those of Bcl-2." J Cell Biol 128(6): 1173-1184.

Yoo, H. Y., A. Shevchenko, A. Shevchenko and W. G. Dunphy (2004). "Mcm2 is a direct substrate of ATM and ATR during DNA damage and DNA replication checkpoint responses." J Biol Chem 279(51): 53353-53364.

Yoshizawa-Sugata, N. and H. Masai (2007). "Human Tim/Timeless-interacting protein, Tipin, is required for efficient progression of $\mathrm{S}$ phase and DNA replication checkpoint." $\mathrm{J}$ Biol Chem 282(4): 2729-2740.

Zeman, M. K. and K. A. Cimprich (2014). "Causes and consequences of replication stress." Nat Cell Biol 16(1): 2-9.

Zhao, H. and H. Piwnica-Worms (2001). "ATR-Mediated Checkpoint Pathways Regulate Phosphorylation and Activation of Human Chk1." Molecular and Cellular Biology 21(13): 4129-4139.

Zhong, Y., T. Nellimoottil, J. M. Peace, S. R. Knott, S. K. Villwock, J. M. Yee, J. M. Jancuska, S. Rege, M. Tecklenburg, R. A. Sclafani, S. Tavare and O. M. Aparicio (2013). "The level of origin firing inversely affects the rate of replication fork progression." J Cell Biol 201(3): 373383.

Zilfou, J. T. and S. W. Lowe (2009). "Tumor Suppressive Functions of p53." Cold Spring Harbor Perspectives in Biology 1(5): a001883. 


\section{Acknowledgements}

First and foremost, I would like to thank my supervisor, Prof. Dr. Matthias Dobbelstein for giving me the opportunity to work in his lab. Thank you very much Matthias! Indeed it has been such a great learning experience to work together with you. My fascination for science and cancer research has only grown more profound over the years under your guidance. Thank you for all the scientific discussions, the brainstorming sessions and your constant support in times of distress. Without your encouragement, this project would not have been possible.

I would also like to sincerely thank my committee members, Dr.Wilfried Kramer and Prof.Dr. Holger Reichardt. Thank you for your time, helpful advice and insights into the project during the committee meetings.

Doing a PhD in Germany would not have been possible without Dr. Steffen Burkhardt and Kerstin Grüniger. Thank you for all your help during these 5 years. It gives me immense pleasure and pride to be associated with the IMPRS Molecular Biology program.

Many thanks to all those people who contributed to this work: Dr. Ann Parplys (Hamburg) for teaching the fiber assay and sharing data. Yizhu and Ina, thank you for helping me out with the fiber assays, with my never ending doubts and questions! Christin, thank you very much, for all the technical support and for sharing data.

Heartfelt thanks to all the former and current lab members of Molecular Oncology. Some names deserve a special mention...

...Frederik Köpper, for taking me as your lab rotation student way back in 2010 ! It has been a long way since then. Thank you for teaching me how to do an experiment, for your discussions and suggestions in my project and for your kind help to correct my thesis!

....Franzi, Magda, Uli, Priyanka and Sonja, for sharing all my highs and lows, for all the laughs in the office, and for all the support professionally and personally throughout my $\mathrm{PhD}$...you have been a great team to work with! My time here would not have been so enjoyable if it weren't for you all!

....Antje, Cathrin, Christin, Kamila and Karola, without your technical support, the lab would not run so smoothly! Thank you for everything!

....Xin, Meike, Leonie and Shelley for providing the best small lab environment that I could ask for!! 
....Anusha, you have only been there for 6 months, but these were the most crucial ones for me. Thank you for comforting me whenever I started to doubt myself, thank you for all the coffee times and thanks for listening to me ramble about 'which sentence looks better'!

Now, to thank all those people who have been a part of my journey right from the beginning. Probably oxford dictionary should have a new synonym for thank you, because for these people that I am going to write about, a mere thank you does not do justice to what I feel for them and what they have done for me! I am out of words, but I will try to do my best! So, walking down the memory lane, in chronological and alphabetical order, here it is:

...Aki and Chats, we came together in September 2009, it's been 5.5 years since then, and it's been one rollercoaster ride that we have had! My first year in Germany would not have been so memorable for all the right and wrong reasons if it weren't for you guys. From being a hopeless and clueless bunch of girls, to mature, grown up individuals, we have come a long way together. Thank you for sharing my happiness and sadness, thank you for motivating me every time, thank you being there in my life. I am truly lucky to have found you! I love you girls! Three cheers to us! Zameer how I can I forget you! Thank you for giving us the best time of our lives here in Germany!

...Kundan, Heena and Vinita, you have been my greatest support system here. I am certain that I will fall short of words to describe my gratitude to you. Undoubtedly, life in Göttingen would be impossible without you.

...Kundan, you are one of the most jovial, helpful and intelligent person that I have come across. I consider myself extremely blessed to have found such an amazing friend and a guide like you! Many a times, I have drawn inspiration from your practicalities in life. Thank you for the innumerable number of laughs, for your constant encouragement and cheer whenever things have gone awry and mostly, thank you for your support and friendship. This one I will cherish for life!

....Heena, I have known you for 8 years now, and if something has changed, it has to be that our friendship has grown stronger and deeper over the years. I feel a sense of security with you. There have been many times when you have understood without me having to say anything. Thank you for believing in me and making me believe in myself! Thank you for being there in my life!

...Vinita, 'schwester'! I think this word, tiny as it may sound, pretty much sums up the equation between us, so understanding and always ready to hear me out and motivate me, you have been a pillar of support that I could lean on, anytime. Thank you for being the best younger sister that I always wished to have!

Ankit, Arnab, Avani, Pawan, Soham and Upasana thank you for your care, love and support during all these years. Your constant reassurance has made me fight all the odds and come out stronger! Thank you for being a part of my journey! Thank you for your friendship. 
Panchi, you came, you saw you conquered our hearts! Thank you for being who you are, thank you for your friendship and love!

This acknowledgement would be incomplete if it weren't for awesome foursome - Mansi, Sheenam and Shruti. Even though we have not been together these five years, distance has only made our hearts grow fonder of each other. We have come a long way, and if it were not for you three girls, I would not even be writing this piece here. You are an integral part of me and my life, we have seen and experienced life together, and you complete me. Thank you!! Shruti, you deserve a special mention. Thank you so much for reading my thesis time and again, and for listening to my constant rants through these two months! Love you girls! Raashi, we have gone through this journey of ups and downs in our $\mathrm{PhD}$ almost together. You have been there for me always, through my good times and bad. Thank you for being there whenever I needed a shoulder to lean on, even if geographically we were separated by miles! Cheers to us for having survived this journey! Sudarshan, thank you for supporting me through my tough times all these years!

Now time to thank my family, Amma, Appa and Kripa, this thesis is for you! For all those continuous sleepless nights that I gave you, here it is! Amma and Appa, thank you for giving me the perfect combination of genes - scientific thinking and creativity. Appa, I have always looked up to you, your hardwork and sincerity has been my source of inspiration all through these years. You have taught me what it is to fight the odds for something that you truly believe in. Amma, you have made me fearless, always been there to listen to me, always wiped my tears and encouraged me to pull my socks up and continue fighting. Thank you for making me the fighter that I am today. Kripa, you have been my best friend throughout. Whether it was telling mom and dad that I flunked in my exam or encouraging me to study further, you have been there with me through thick and thin. Thank you 'seester'! I love you all a lot! Swarup 'jeej', the newest addition to our family, thank you for your constant support, love and encouragement! Next pit stop - US of A!! Fingers crossed!

I also want to take this opportunity and thank all my extended family members who have always stood by me through these times and constantly encouraged me to perform better.

Last but not the least, I would like to thank nature itself for creating something so beautiful, for creating us so that we could appreciate and understand what is around us. Big thanks to biology for being ever so fascinating and beautiful. 


\section{Curriculum vitae}

\section{Personal Details}

Name

Veena Jagannathan

Address

Institute for Molecular Oncology, GZMB, Justus-von-Liebig 11, 37077 Göttingen, Germany

Date of Birth $10^{\text {th }}$ February 1989

Nationality

USA

Phone

0049-551-39-13842

email

vjagann@gwdg.de

\section{Education}

\begin{tabular}{ll}
\hline 2011-Present & $\begin{array}{l}\text { PhD in Molecular Biology, IMPRS, Institute } \\
\text { for Molecular Oncology, University of } \\
\text { Göttingen, Göttingen, Germany }\end{array}$ \\
\hline $2009-2011$ & M.Sc. in Molecular Biology, International Max \\
& Planck Research School (IMPRS), Göttingen, \\
& Germany, Grade of Master's examinations: \\
& $1.33=$ A (excellent) \\
\hline $2006-2009$ & B.Sc. (H) Biochemistry, University of Delhi, \\
& India, First Division
\end{tabular}

Projects and Research

\begin{tabular}{ll}
\hline $10 / 2011-$ Present & The phosphatase MKP1 as a target to \\
& enhance replicative stress and apoptosis in \\
tumor cells (PhD Project). & Institute for Molecular Oncology, GZMB, \\
& University of Göttingen, Göttingen, Germany \\
\hline $05 / 2010-06 / 2010$ & Identification of sub-cellular localization of \\
& endogenous E3 ubiquitin ligases -WWP2 \\
& and WWP1 in mouse neurons. Max Planck \\
& Institute for Experimental Medicine, \\
& Göttingen, Germany \\
\hline $03 / 2010-04 / 2010$ & Monitoring the interaction between SlyD and \\
& Signal Recognition Particle (SRP) using \\
Isothermal Thermal Calorimetry. & Max Planck Institute for Biophysical \\
& Chemistry, Göttingen, Germany. \\
\hline $01 / 2010-02 / 2010$ & The role of kinase MAPKAPK2 in DNA \\
damage response to \\
Gemcitabine treatment.
\end{tabular}


Institute for Molecular Oncology, GZMB, University of Göttingen, Göttingen, Germany

$2008-2010$

To monitor the growth kinetics Mycobaterium smegmatis as a function of different media, the characterization of dps 2 promoter by qualitative $\beta$-gal assay, and to study the GPL profile of Mycobacterium smegmatis in different media'

Molecular Biophysics Unit, Indian Institute of Science, Bangalore, India.

\section{Awards and Scholarships}

\begin{tabular}{ll}
\hline June 2011-May 2014 & $\begin{array}{l}\text { Graduiertenkollegs1034 (GRK1034) } \\
\text { Stipendium, University of Göttingen, } \\
\text { Germany for carrying out doctoral thesis. }\end{array}$ \\
\hline March 2011 & $\begin{array}{l}\text { STSM award for research training in } \\
\text { Stockholm University for a period of two } \\
\text { weeks }\end{array}$ \\
\hline $09 / 2009-08 / 2010$ & Stipend of the Excellence Foundation for the \\
& Promotion of the Max Planck Society. \\
\hline $2008-2009$ & Certificate of merit, Awarded Medal prize for \\
& highest marks in Bsc (H) Biochemistry, and \\
& third rank in Delhi University, India \\
\hline $05 / 2008-06 / 2008$ & Awarded Summer Research Fellowship, \\
& sponsored by IASc (Bangalore), INSA (New \\
& Delhi) and NASI (Allahabad) \\
\hline $2007-2008$ & Certificate of Merit for securing third position \\
& in South Delhi Campus in University \\
& Examination
\end{tabular}

\section{Conferences and Symposia's}

\begin{tabular}{ll}
\hline $09 / 2013$ & $\begin{array}{l}\text { Eukaryotic DNA Replication \& Genome } \\
\text { Maintenance, Cold Spring Harbor } \\
\text { Laboratories, New York, USA }\end{array}$ \\
\hline $09 / 2012$ & Horizons in Molecular Biology, Gottingen, \\
& Germany \\
\hline $09 / 2011$ & Horizons in Molecular Biology, Gottingen, \\
& Germany, awarded third best poster prize.
\end{tabular}




\section{Teaching Experience}

\begin{tabular}{ll}
\hline 2014 & Lab Rotation Student: Supervised a lab \\
& rotation student from the IMPRS Molecular \\
& Biology Program from 01/2014 - 02/2014. \\
& The research project was entitled 'The Role \\
& of MAP Kinase Phosphatase 1 (MKP1) in the \\
& Sensitization of U2OS Cancer Cells to the \\
& Nucleoside Analogue Gemcitabine'. \\
\hline $2012-2014$ & Conducted Method/Practical courses in \\
& Luminometry for graduate students from \\
& GGNB and IMPRS programs. \\
\hline $2012-2013$ & Interactive tutorials for Masters Students, part \\
& of the IMPRS Molecular Biology. \\
\hline 2012 & Lab Rotation Student: Supervised a lab \\
& rotation student from the Molecular Medicine \\
& Program from 07/2012 - 09/2012. The \\
& research project was entitled 'Inhibition of the \\
& Mitogen Activated Protein Kinase-1 in U2OS \\
& Cells'.
\end{tabular}

\section{Skills}

- Proficient in basic molecular biology techniques and the routine quantitative and structural proteomics workflow.

- Languages: Hindi (Native), English (Native), Tamil (Native) German (Basic level of working proficiency). 